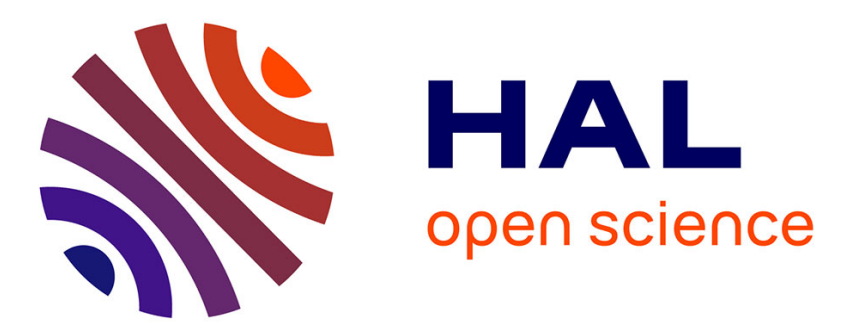

\title{
Mean Geometry for 2D random fields: level perimeter and level total curvature integrals
}

Hermine Biermé, Agnès Desolneux

\section{To cite this version:}

Hermine Biermé, Agnès Desolneux. Mean Geometry for 2D random fields: level perimeter and level total curvature integrals. Annals of Applied Probability, 2020, 30 (2), pp.561-607. 10.1214/19AAP1508 . hal-01370902v5

\section{HAL Id: hal-01370902 \\ https://hal.science/hal-01370902v5}

Submitted on 10 Apr 2019

HAL is a multi-disciplinary open access archive for the deposit and dissemination of scientific research documents, whether they are published or not. The documents may come from teaching and research institutions in France or abroad, or from public or private research centers.
L'archive ouverte pluridisciplinaire HAL, est destinée au dépôt et à la diffusion de documents scientifiques de niveau recherche, publiés ou non, émanant des établissements d'enseignement et de recherche français ou étrangers, des laboratoires publics ou privés. 


\title{
Mean Geometry for 2D random fields: Level perimeter and level total curvature integrals
}

\author{
Hermine Biermé \\ Hermine Biermé \\ LMA (UMR CNRS 7348) \\ Université de Poitiers, \\ 11 bd Marie et Pierre Curie, \\ 86962 Chasseneuil, France \\ e-mail: hermine.bierme@math.univ-poitiers.fr \\ and \\ Agnès Desolneux \\ Agnès Desolneux \\ CNRS, CMLA (UMR 8536), \\ Université Paris-Saclay, ENS Cachan, \\ 61 avenue du Président Wilson, \\ 94235 CACHAN cedex, France \\ e-mail: agnes.desolneux@cmla.ens-cachan.fr
}

\begin{abstract}
We introduce the level perimeter integral and the total curvature integral associated with a real-valued function $f$ defined on the plane $\mathbb{R}^{2}$, as integrals allowing to compute the perimeter of the excursion set of $f$ above level $t$ and the total (signed) curvature of its boundary for almost every level $t$. Thanks to the Gauss-Bonnet theorem, the total curvature is directly related to the Euler Characteristic of the excursion set. We show that the level perimeter and the total curvature integrals can be computed in two different frameworks: smooth (at least $C^{2}$ ) functions and piecewise constant functions (also called here elementary functions). Considering 2D random fields (in particular shot noise random fields), we compute their mean perimeter and total curvature integrals, and this provides new "explicit" computations of the mean perimeter and Euler Characteristic densities of excursion sets, beyond the Gaussian framework: for piecewise constant shot noise random fields we give some examples of completely explicit formulas, and for smooth shot noise random fields the provided examples are only partly explicit, since the formulas are given under the form of integrals of some special functions.
\end{abstract}

MSC 2010 subject classifications: Primary 60G60, 60G17, 60D05, 60E10, 26B15; secondary 60G10, 60E07, 62M40.

Keywords and phrases: Perimeter, Total curvature, Gauss-Bonnet Theorem, Euler Characteristic, excursion sets, stationary random field, shot noise random field, Gaussian random field, persistent homology.

\section{Contents}

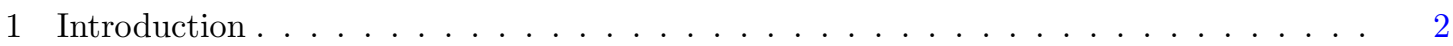

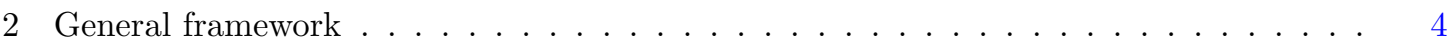

2.1 Sets of finite perimeter and finite total curvature . . . . . . . . . . . . . . 4

2.2 Level integrals for excursion sets . . . . . . . . . . . . . . . . . 7

3 Level integrals of smooth functions and random fields . . . . . . . . . . . . . 8

3.1 The case of smooth functions $\ldots \ldots \ldots \ldots \ldots$ 
3.2 A general result for smooth stationary random fields . . . . . . . . . . . . . 11

3.3 Smooth shot noise random fields . . . . . . . . . . . . . . . . 18

4 Elementary functions and elementary shot noise random fields . . . . . . . . . . . . . 24

4.1 Elementary functions . . . . . . . . . . . . . . . . . . . 24

4.2 Level perimeter and level total curvature of an elementary shot noise random field 29

4.3 Explicit computations . . . . . . . . . . . . . . . . 36

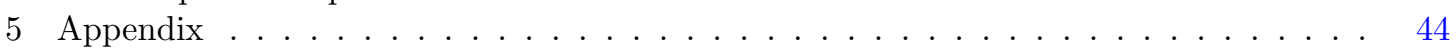

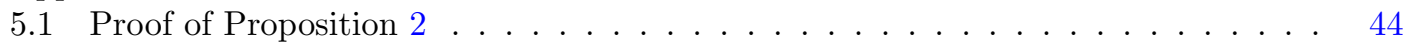

5.2 Proof of Proposition $3 \ldots \ldots \ldots \ldots \ldots$

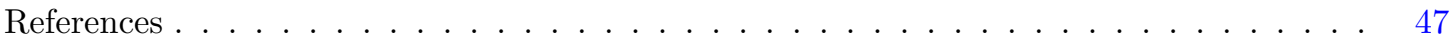

\section{Introduction}

Considering a real-valued stationary 2-dimensional random field $X=(X(x))_{x \in \mathbb{R}^{2}}$, defined on a complete probability space $(\Omega, \mathcal{A}, \mathbb{P})$, we are interested in statistically describing the geometry of its excursions sets, defined for $t \in \mathbb{R}$ by

$$
E_{X}(t):=\{X \geq t\} \subset \mathbb{R}^{2},
$$

in a given bounded open subset of $\mathbb{R}^{2}$. In the following $U$ will denote an open bounded set or simply $\mathbb{R}^{2}$ when not bounded. We will focus on $E_{X}(t) \cap U=\{x \in U ; X(x) \geq t\}$ as well as $\partial E_{X}(t) \cap U$ its boundary trace on $U$, where as usual $\partial E_{X}(t)=\overline{E_{X}(t)} \backslash E_{X}^{o}(t)$.

In view of the measurability of $X$, its excursion sets $E_{X}(t)$ are a.s. Borel sets for all level $t \in \mathbb{R}$. When moreover $X$ is a.s. upper semi-continuous, these random sets are a.s. closed (see [30]) and therefore, for bounded $U$, the set $E_{X}(t) \cap \bar{U}$ is a compact random set. In dimension 2 , the geometry of a compact "nice" set $K \subset \mathbb{R}^{2}$ with piecewise $C^{2}$ boundary $\partial K$ may be described by three functionals: its area $\mathcal{L}(K)$, where $\mathcal{L}$ is the Lebesgue measure on $\mathbb{R}^{2}$, its perimeter $\operatorname{Per}(K)=\mathcal{H}^{1}(\partial K)$, where $\mathcal{H}^{1}$ is the one-dimensional Hausdorff measure, and its Euler Characteristic $\chi(K)$ that counts the number of connected components minus the number of holes. According to the Gauss-Bonnet Theorem, when $\partial K$ is a disjoint finite union of closed curves, $\chi(K)$ is also equal to $\frac{1}{2 \pi} \mathrm{TC}(\partial K)$, with $\mathrm{TC}(\partial K)$ the total curvature of the positively oriented curve $\partial K$ (see precise definitions and statements in Definition 2 and Theorem 1). Let us notice that these geometrical features are also used with different conventions according to the setting. For instance, in convex geometry, for $K$ a convex body, intrinsic volumes, respectively Minkowski's functionals, are defined by $V_{0}(K)=\chi(K)=1, V_{1}(K)=\frac{1}{2} \mathcal{H}^{1}(\partial K)$ and $V_{2}(K)=$ $\mathcal{L}(K)$, respectively $W_{2}(K)=\frac{1}{2} \mathrm{TC}(\partial K)=\pi, W_{1}(K)=\frac{1}{2} \mathcal{H}^{1}(\partial K)$ and $W_{0}(K)=\mathcal{L}(K)$ (see [34]), while in differential geometry when $K$ is a compact 2-dimensional submanifold with $C^{2}$ smooth boundary, Lipschitz Killing curvatures of $K$ are defined by $C_{0}(K)=\frac{1}{2 \pi} \mathrm{TC}(\partial K), C_{1}(K)=$ $\frac{1}{2} \mathcal{H}^{1}(\partial K)$ and $C_{2}(K)=\mathcal{L}(K)$ and may be extended to sets with positive reach (see [35]).

When considering stationary random sets, it is natural to define corresponding mean density functionals. They are usually defined considering the limit behavior of a rescaled observation through a large window, say $r U$, for $r$ large. Actually, this procedure allows to remove boundary effects. We adopt a similar point of view in this paper, by removing boundary effect, using a window $U$ that is open. Hence, for a bounded open $U$ we will focus on the mean area $\mathbb{E}\left(\mathcal{L}\left(E_{X}(t) \cap U\right)\right)$, the mean perimeter $\mathbb{E}\left(\operatorname{Per}\left(E_{X}(t), U\right)\right)$, and on the mean total curvature $\mathbb{E}\left(\mathrm{TC}\left(\partial E_{X}(t), U\right)\right)$ of excursion sets. Densities will then clearly appear as

$$
\begin{gathered}
\mathbb{E}\left(\mathcal{L}\left(E_{X}(t) \cap U\right)\right)=\overline{\mathcal{L}\left(E_{X}(t)\right)} \mathcal{L}(U), \quad \mathbb{E}\left(\operatorname{Per}\left(E_{X}(t), U\right)\right)=\overline{\operatorname{Per}\left(E_{X}(t)\right)} \mathcal{L}(U) \\
\text { and } \quad \mathbb{E}\left(\mathrm{TC}\left(\partial E_{X}(t), U\right)\right)=\overline{\operatorname{TC}\left(E_{X}(t)\right)} \mathcal{L}(U) .
\end{gathered}
$$


As far as stationarity is involved, the mean area is not hard to find since

$$
\begin{aligned}
\mathbb{E}\left(\mathcal{L}\left(E_{X}(t) \cap U\right)\right) & =\int_{U} \mathbb{E}\left(\mathbf{1}_{X(x) \geq t}\right) d x \\
& =\mathcal{L}(U) \mathbb{P}(X(0) \geq t) .
\end{aligned}
$$

It follows that an exact formula can be set up as soon as the distribution of $X(0)$ (that is the same as any $X(x)$ by stationarity) is known and $\overline{\mathcal{L}\left(E_{X}(t)\right)}=\mathbb{P}(X(0) \geq t)$. Now establishing formulas for the mean perimeter and the mean total curvature or Euler Characteristic is more difficult and requires additional assumptions on the field. Computing the Euler Characteristic of excursion sets of random fields is a problem that has received much attention. Indeed, in many applications, the Euler Characteristic is a very useful index of the geometry of the field, as explained for instance in the review paper of R. Adler [2], or in the papers of K. Worsley [37] or [38] where applications in astrophysics or in brain imaging are mentioned.

Despite its "global" definition (the number of connected components minus the number of holes), the Euler Characteristic of an excursion set is in fact a purely local quantity related, by Morse theory, to the number of critical points of $X$ in $U$, or, by the Gauss-Bonnet theorem, to the total curvature of the boundary of the excursion set. Here, we will extensively use this second equivalence to obtain explicit computations of the mean Euler Characteristic density of the excursion sets of some random fields.

In the framework of Gaussian random fields, the first equivalence is usually used. For stationary isotropic Gaussian random fields, an explicit formula for any level $t$ may be obtained for the expectation of the Euler Characteristic density, only depending on the variance and on the second spectral moment of the field. This is an important result with many statistical applications. In particular, for large levels $t$, the Euler Characteristic gives a good approximation of the probability that the supremum of the field is greater than $t$ and can therefore be used as a p-value: this is the Euler Characteristic heuristic (see [5] for instance). In a "tour de force", a Central Limit Theorem has recently been established in [19] that proves the accuracy of the estimation over only one sample path as the size of the observation is growing. There are also some interesting results apart from the Gaussian framework for $\chi^{2}, F$ and $t$-fields [37] as well as stable [3] or infinitely divisible random fields [4] for instance. A test of Gaussianity can therefore be set up using the Euler Characteristic of the level sets as proposed in [16]. However, most of the general results rely on strong smoothness regularity assumptions and on conditional distribution densities that are often difficult to evaluate for non-Gaussian fields.

Now, in this paper, we will be particularly interested in another family of infinitely divisible random fields, that are not Gaussian, namely the shot noise random fields. A shot noise random field is defined on $\mathbb{R}^{2}$ by

$$
\forall x \in \mathbb{R}^{2}, \quad X(x)=\sum_{i} g_{m_{i}}\left(x-x_{i}\right),
$$

where the $x_{i}$ are the points of an homogeneous Poisson point process of intensity $\lambda$ in $\mathbb{R}^{2}$, and the $m_{i}$ are "marks", independent of the Poisson point process. Such fields allow explicit computations and may appear, in view of their asymptotic normality in high intensity [21], as a bridge between the Gaussian setting and the discrete models of stochastic geometry such as the Boolean ones [34]. Several results for the computation of the perimeter were obtained in our previous paper [11]. Since the study for all level $t$ is often difficult, we extend here our point of view of working in a weak framework by considering the geometric quantities as functions of the level $t$. Hence quantities of interest will be given, when it makes sense, by the mean level perimeter integral $\mathbb{E}\left(\operatorname{LP}_{X}(h, U)\right)$ and the mean level total curvature integral $\mathbb{E}\left(\operatorname{LTC}_{X}(h, U)\right)$ of $X$, where the level perimeter integral and the level total curvature integral are defined, for $h$ a continuous bounded 
function on $\mathbb{R}$, by

$$
\operatorname{LP}_{X}(h, U):=\int_{\mathbb{R}} h(t) \operatorname{Per}\left(E_{X}(t), U\right) d t
$$

and

$$
\operatorname{LTC}_{X}(h, U):=\int_{\mathbb{R}} h(t) \mathrm{TC}\left(\partial E_{X}(t), U\right) d t .
$$

Let us remark that this allows us to get informations on the mean geometry of excursion sets for almost every level $t$, and hence to give insights on their evolution according to the level. We will in particular be interested in the case of functions $h_{u}: t \mapsto e^{i u t}$ with $u \in \mathbb{R}$, computing then the Fourier transform of $t \mapsto \operatorname{Per}\left(E_{X}(t), U\right)$ and $t \mapsto \mathrm{TC}\left(\partial E_{X}(t), U\right)$. This setting will also allow us to recover and generalize some important results established in the two different frameworks of Boolean models in stochastic geometry and of smooth Gaussian random fields.

In Section 2 we propose a general definition of the level perimeter integral and of the level total curvature integral of a function, that allows to compute the perimeter and the total curvature (and therefore the Euler Characteristic) of its excursion sets for almost every level. Section 3 is devoted to the results on smooth (random) functions. In particular our weak framework allows us to get formulas for some isotropic fields, recovering known Gaussian results. We introduce then elementary functions as a particular case of piecewise constant functions (with piecewise smooth discontinuity set) in Section 4 and compute their level perimeter and total curvature integral. We give explicit expressions for elementary shot noise random fields, where the functions $g_{m_{i}}$ are elementary functions. This allows us to generalize results of the literature (about the Boolean model [28], or about "random configurations" [15]).

Let us finally emphasize that we have made here the deliberate choice of not working in the weakest possible functional framework. Our goal is to work else with smooth or with piecewise constant functions (like the indicator function of a set having a piecewise $C^{2}$ boundary for instance). But we believe some of our results can be extended to functions with a weakest regularity. Let us also mention the recent work of R. Lachièze-Rey in [25] and [24] that relates the Euler Characteristic to the three-point joint distribution of the random field. And also the paper [23] where R. Lachièze-Rey gives formulas for the Euler Characteristic of isotropic shot noise random field that are a.s. Morse functions.

\section{General framework}

\subsection{Sets of finite perimeter and finite total curvature}

We consider a Borel set $E$ of $\mathbb{R}^{2}$ and an open set $U \subset \mathbb{R}^{2}$. We assume that the indicator function $\mathbf{1}_{E}$ is a function in $S B V(U)$. Let us recall (see [7]) that a function $f$ belongs to $S B V(U)$, the space of special functions of bounded variation in $U$ if $f \in L^{1}(U)$ and has its distributional derivative representable by a finite Radon measure in $U$ that is

$$
\int_{U} f(x) \frac{\partial \phi}{\partial x_{l}}(x) d x=-\int_{U} \phi(x) D_{l} f(d x) \quad \forall \phi \in C_{c}^{1}(U, \mathbb{R}), \quad \forall l=1,2
$$

for some $\mathbb{R}^{2}$-valued measure $D f=\left(D_{1} f, D_{2} f\right)$, such that

$$
D f=\nabla f \mathcal{L}+\left(f^{+}-f^{-}\right) \nu_{f} \mathcal{H}^{1} \angle J_{f},
$$


where $\nabla f \mathcal{L}$ is the absolutely continuous part of the Radon measure $D f$ with respect to the Lebesgue measure $\mathcal{L}$ and $\left(f^{+}-f^{-}\right) \nu_{f} \mathcal{H}^{1} \angle J_{f}$ is the jump part of $D f$, with $J_{f}$ the set of approximate jump points of $f$. The set $J_{f}$ is included in the approximate discontinuity set $\mathcal{S}_{f}$ that is the set of points where $f$ is not approximately continuous (see [7] Proposition 3.64 p.160 and Theorem 3.83 p.176 for more details).

This framework, used in our previous paper [11], is convenient to define the perimeter of a set $E$ in $U$ such that $\mathbf{1}_{E} \in S B V(U)$ as

$$
\operatorname{Per}(E, U):=\left\|D \mathbf{1}_{E}\right\|(U)=\sup \left\{\int_{U} \mathbf{1}_{E} \operatorname{div} \varphi d x \mid \varphi \in C_{c}^{1}\left(U, \mathbb{R}^{2}\right),\|\varphi\|_{\infty} \leq 1\right\}<+\infty
$$

It follows that, denoting by $\mathcal{S}_{\mathbf{1}_{E}}$ the approximate discontinuity set of $\mathbf{1}_{E}$, the set $\mathcal{S}_{\mathbf{1}_{E}} \cap U$ (its trace in $U)$ is included in $\partial E \cap U$ the boundary of $E$ in $U$. In order to get information linked with length and Euler Characteristic we make the stronger assumption that $\partial E \cap U$ coincides with $\mathcal{S}_{\mathbf{1}_{E}} \cap U$ (which is equivalent to say that the discontinuity points are exactly the approximate discontinuity points in $U$ ), and is a piecewise $C^{2}$ plane curve.

Let us recall here some basic facts and definitions about plane curves, following [17]. When $\Gamma$ is a piecewise $C^{2}$ simple oriented curve (possibly closed) we define a regular point or a corner point $x$ of $\Gamma$ by the following properties:

- regular point: one can find an arc-length $C^{2}$ parametrization $\gamma:(0, \varepsilon) \rightarrow \Gamma$ with $x=\gamma(s)$ for some $s \in(0, \varepsilon)$, with $\varepsilon>0$, and a normal vector $\nu_{\Gamma}(x)=\gamma^{\prime}(s)^{\perp} \in S^{1}$ with $\gamma^{\prime}(s)^{\perp}$ the $+\frac{\pi}{2}$ rotation of the tangent vector $\gamma^{\prime}(s)$. The normal cone (defined for sets with positive reach [35]) of $\Gamma$ at $x$ is given by $\operatorname{Nor}(\Gamma, x)=\left\{-\nu_{\Gamma}(x)\right\}$. The signed curvature $\kappa_{\Gamma}(x)$ of $\Gamma$ at $x=\gamma(s)$ is then defined as

$$
\kappa_{\Gamma}(x)=\left\langle\gamma^{\prime \prime}(s), \nu_{\Gamma}(x)\right\rangle,
$$

where $\langle\cdot, \cdot\rangle$ is the usual Euclidean scalar product on $\mathbb{R}^{2}$. Note that since $\gamma$ is an arc-length parametrization we have $\mathcal{H}^{1}(\gamma(0, \varepsilon))=\varepsilon$.

- corner point: one can find a simple continuous arc-length parametrization $\gamma:(-\varepsilon, \varepsilon) \rightarrow \Gamma$ such that $x=\gamma(0)$ with $\gamma$ being $C^{2}$ on $(-\varepsilon, \varepsilon) \backslash\{0\}$ and $\gamma^{\prime}$ admits limits $\gamma^{\prime}\left(0^{-}\right) \in S^{1}$ and $\gamma^{\prime}\left(0^{+}\right) \in S^{1}$ at 0 , with $\nu_{\Gamma}^{-}(x):=\gamma^{\prime}\left(0^{-}\right)^{\perp}$ and $\nu_{\Gamma}^{+}(x):=\gamma^{\prime}\left(0^{+}\right)^{\perp}$ linearly independent (no "cusp") in $S^{1}$ such that the normal cone of $\Gamma$ at $x$ is given by $\operatorname{Nor}(\Gamma, x)=\left\{-p \nu_{\Gamma}^{-}(x)-\right.$ $\left.q \nu_{\Gamma}^{+}(x) ; p, q \geq 0\right\} \cap S^{1}$. We then define $\beta_{\Gamma}(x) \in(0, \pi)$ the angle of the cone Nor $(\Gamma, x)$, corresponding to the size of the jump of $\nu_{\Gamma}$ at point $x$ and $\alpha_{\Gamma}(x)= \pm \beta_{\Gamma}(x) \in(-\pi, \pi)$ the turning angle at $x$, where the sign is given according to the orientation of the curve. Note that we also have $\mathcal{H}^{1}(\gamma((-\varepsilon, \varepsilon)))=2 \varepsilon$.

We denote by $\mathcal{R}_{\Gamma}$ the set of regular points and by $\mathcal{C}_{\Gamma}$ the set of corner points of the curve $\Gamma$. In the following we will also denote by $\mathcal{H}^{0}$ the zero-dimensional Hausdorff measure, that is just the counting measure.

Definition 1 (Piecewise regular curve). We say that $\Gamma$ is a piecewise regular curve if it is a simple oriented curve given by a finite union of piecewise $C^{2}$ disjoint Jordan curves (i.e. simple closed curves) with a finite number of corner points such that $\Gamma=\mathcal{R}_{\Gamma} \cup \mathcal{C}_{\Gamma}$. It has a finite length given by $\mathcal{H}^{1}(\Gamma)=\mathcal{H}^{1}\left(\mathcal{R}_{\Gamma}\right)$ and a finite absolute total curvature on any Borel set $U \subset \mathbb{R}^{2}$, given by

$$
\operatorname{TaC}(\Gamma, U)=\int_{\mathcal{R}_{\Gamma} \cap U}\left|\kappa_{\Gamma}(x)\right| \mathcal{H}^{1}(d x)+\sum_{x \in \mathcal{C}_{\Gamma} \cap U}\left|\alpha_{\Gamma}(x)\right|<+\infty .
$$

Notice that the definition of $\mathrm{TaC}$ is the same as the one introduced by Milnor in [29] for closed curves. But here, in this work, we will pay a particular attention to the signed total curvature, and not to its absolute value. 
Definition 2 (Elementary set, Perimeter and Total curvature). We say that a Borel set $E \subset \mathbb{R}^{2}$ is an elementary set if $\partial E$ is a piecewise regular curve (Definition 1) positively oriented in such a way that the normals are oriented towards $E$. It follows that, for any open bounded set $U \subset \mathbb{R}^{2}$, then $\mathbf{1}_{E} \in S B V(U)$, the length of the curve $\partial E \cap U$ is given by

$$
\mathcal{H}^{1}(\partial E \cap U)=\mathcal{H}^{1}\left(\mathcal{R}_{\partial E} \cap U\right)=\operatorname{Per}(E, U)<+\infty,
$$

and its total curvature in $U$ is

$$
\mathrm{TC}(\partial E, U):=\int_{\mathcal{R}_{\partial E} \cap U} \kappa_{\partial E}(x) \mathcal{H}^{1}(d x)+\sum_{x \in \mathcal{C}_{\partial E} \cap U} \alpha_{\partial E}(x) \in \mathbb{R} .
$$

The link between $\mathcal{H}^{1}(\partial E \cap U)$ and $\operatorname{Per}(E, U)$ follows from Gauss-Green Theorem (see Section 3.3 of [7]). Our definition of total curvature is the same as the one of Santaló in [31], Chapter 7. The total curvature is intrinsic, it doesn't depend on the parametrization of the curve. But it depends on its orientation: if we reverse the orientation of the curve then its total curvature is changed into its opposite. In particular, when $E$ is an elementary set, since $\partial E=\partial E^{c}$, its complement $E^{c}=\mathbb{R}^{2} \backslash E$ is also an elementary set, with for all $U$ open bounded set:

$$
\operatorname{Per}\left(E^{c}, U\right)=\operatorname{Per}(E, U), \operatorname{TaC}\left(\partial E^{c}, U\right)=\operatorname{TaC}(\partial E, U) \text { and } \operatorname{TC}\left(\partial E^{c}, U\right)=-\operatorname{TC}(\partial E, U) .
$$

On Figure 1 we give examples of sets that are or are not elementary according to our definition.

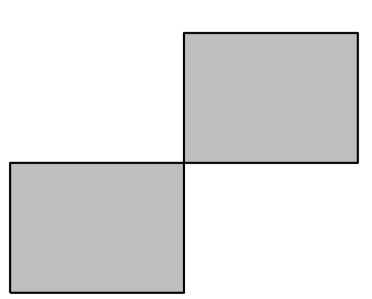

Not elementary

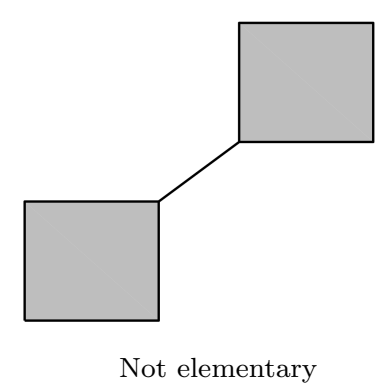

Not elementary

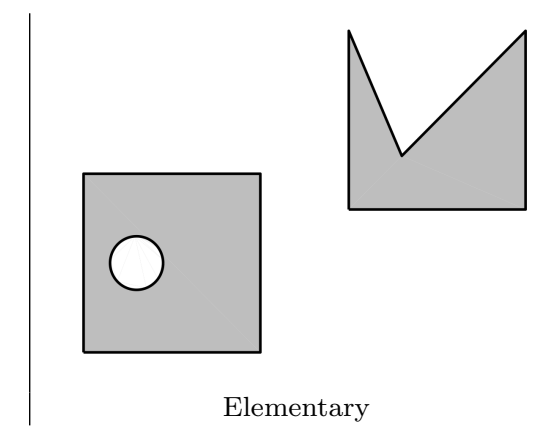

Elementary

FIG 1. Left: two sets that are not elementary sets according to Definition 2: the boundary of the first set has one multiple point; for the second set, its boundary is not the union of disjoint piecewise $C^{2}$ Jordan curves. Right: an elementary set.

The link between the total curvature and the Euler Characteristic is given by the GaussBonnet theorem. Since the boundary of an elementary set is a finite disjoint union of Jordan piecewise $C^{2}$ curves we can apply Gauss-Bonnet Theorem (as stated in [17] p.274) for a regular region.

Theorem 1 (Gauss-Bonnet Theorem). Let E be a bounded elementary set that is a regular region (meaning that $E=\stackrel{\bar{o}}{E}$ ), then the Euler Characteristic of $E$ is given by

$$
\chi(E)=\frac{1}{2 \pi} \mathrm{TC}(\partial E)=\frac{1}{2 \pi}\left(\int_{\mathcal{R}_{\partial E}} \kappa_{\partial E}(x) \mathcal{H}^{1}(d x)+\sum_{x \in \mathcal{C}_{\partial E}} \alpha_{\partial E}(x)\right) .
$$

Remark 1: It is a well-known result of differential geometry of plane curves that the total curvature of any regular simple closed curve is $2 \pi$ or $-2 \pi$ (depending on the orientation of the 
curve). This result is sometimes called Hopf's Umlaufsatz, or also the theorem of turning tangents ([17] p.396).

Remark 2: Note that when $E$ is a bounded elementary set and a regular region, then we have seen that $\partial E=\partial E^{c}$ and $\mathrm{TC}\left(\partial E^{c}, U\right)=-\mathrm{TC}(\partial E, U)$ for any open set $U$ such that $E \subset U$. In contrast, for Euler Characteristic, we have to consider a compact set. For instance, if $V$ is a closed rectangle we can take $V \backslash \stackrel{o}{E}$, and that yields $\chi(V \backslash \stackrel{o}{E})=1-\chi(E)$.

The notion of elementary set is stable under union and intersection provided that the sets are in "generic" position. Indeed, let us introduce the following definition.

Definition 3 (Generic position). Let $E$ and $F$ be two elementary sets. We say that $E$ and $F$ are in generic position if $\partial E \cap \partial F$ is a finite set, included in $\mathcal{R}_{\partial E} \cap \mathcal{R}_{\partial F}$ and if for $x \in \partial E \cap \partial F$, the normals $\nu_{\partial E}(x)$ and $\nu_{\partial F}(x)$ are not colinear.

When $n \geq 3$ we say that $n$ elementary sets $E_{1}, \ldots, E_{n}$ are in generic position if for any subset $J \subset\{1, \ldots, n\}$ with cardinal greater than 3 we have $\cap_{j \in J} \partial E_{j}=\emptyset$ and if each pair of sets are in generic position.

It follows that if $E_{1}, \ldots, E_{n}$ are elementary sets in generic position, then $\cap_{i=1}^{n} E_{i}$ and $\cup_{i=1}^{n} E_{i}$ are also elementary sets.

\subsection{Level integrals for excursion sets}

Definition 4 (Level perimeter and total curvature integrals). Let $U \subset \mathbb{R}^{2}$ be an open set and let $f \in S B V(U)$ be a real-valued special function of bounded variation defined on $U$. For $t \in \mathbb{R}$, we define the excursion set of $f$ for the level $t$ as

$$
E_{f}(t):=\{x \in U ; f(x) \geq t\} \subset \mathbb{R}^{2} .
$$

We assume that for almost every $t \in \mathbb{R}$, the set $E_{f}(t)$ is an elementary set in the sense of Definition 2 and that $t \mapsto \operatorname{TaC}\left(\partial E_{f}(t), U\right)$ is an integrable function on $\mathbb{R}$. We then say that the function $f$ is of finite level total curvature integral on $U$. The level perimeter integral and the level total curvature integral of $f$ are defined for any bounded continuous function $h$ on $\mathbb{R}$ by

$$
\operatorname{LP}_{f}(h, U)=\int_{\mathbb{R}} h(t) \operatorname{Per}\left(E_{f}(t), U\right) d t \quad \text { and } \quad \operatorname{LTC}_{f}(h, U)=\int_{\mathbb{R}} h(t) \mathrm{TC}\left(\partial E_{f}(t), U\right) d t .
$$

We simply denote $V_{f}(U)$ for $\operatorname{LP}_{f}(1, U)\left(=\|D f\|(U)\right.$ by the co-area formula [7]) and $\operatorname{LTC}_{f}(U)$ for $\operatorname{LTC}_{f}(1, U)$.

Let us remark that when $t>\sup _{U} f$, then $E_{f}(t) \cap U=\emptyset$ and therefore $\operatorname{Per}\left(E_{f}(t), U\right)=$ $\mathrm{TC}\left(\partial E_{f}(t), U\right)=0$. On the other hand, when $t \leq \inf _{U} f$, then $E_{f}(t) \cap U=U$, and thus $\partial E_{f}(t) \cap U=$ $\emptyset$. Therefore we also have $\operatorname{Per}\left(E_{f}(t), U\right)=\mathrm{TC}\left(\partial E_{f}(t), U\right)=0$. This shows that the perimeter and total curvature are 0 for levels $t$ outside the range of $f$.

Let us also notice that when $H$ is a $C^{1}$ diffeomorphism on $\mathbb{R}$ with bounded non-negative derivative $h=H^{\prime}$, by a simple change of variable, the function $H \circ f$ is also of special variation and of finite level total curvature integral on $U$ with $E_{H \circ f}(t)=E_{f}\left(H^{-1}(t)\right)$ so that

$$
V_{H \circ f}(U)=\operatorname{LP}_{f}(h, U) \text { and } \operatorname{LTC}_{H \circ f}(U)=\operatorname{LTC}_{f}(h, U) .
$$


Link with Euler Integral. Due to the additivity property of the Euler Characteristic (valid for instance on the class of polyconvex sets, see [14])

$$
\chi(A \cup B)=\chi(A)+\chi(B)-\chi(A \cap B),
$$

it is natural to set up an integration theory with respect to the Euler Characteristic $[26,36]$. However, since $\chi$ is only finitely additive, a careful choice of integrands must be done. This problem was tackled by defining the class of constructible functions [32], then extended by the class of "tame" real-valued functions in [9]. Following this framework, Bobrowski and Borman obtained in [13] the first probabilistic statement about the persistent homology generated by sublevel sets. We briefly recall the definitions used in [13] for comparison with our setting. When $f$ is a real continuous function defined on a compact topological set $S$, it is said to be a tame function if the homotopy types of $E_{f}(t)=\{f \geq t\}$ and $\{f \leq t\}$ change only finitely many times as $t$ varies over $\mathbb{R}$, and the Euler Characteristic of each set is always finite. For such a function, a lower and upper Euler integrals are defined by

$$
\begin{aligned}
& \int_{S} f\lfloor d \chi\rfloor=\int_{0}^{+\infty}(\chi(f \geq t)-\chi(f<-t)) d t \\
& \int_{S} f\lceil d \chi\rceil=\int_{0}^{+\infty}(\chi(f>t)-\chi(f \leq t)) d t,
\end{aligned}
$$

where $\chi(f \geq t)=\chi\left(E_{f}(t)\right), \chi(f<t)=\chi(S)-\chi(f \geq t)$, etc. Note that when $U=(0, T)^{2}$ with some $T>0$, and $S=\bar{U}$, we have $\chi(f \geq t)=\chi(S)=1$ for any $t \leq \min _{S} f$ and thus $t \mapsto \chi(f \geq t)$ is not integrable on $\mathbb{R}$, explaining the above definition of Euler integrals.

In contrast, we can simply define $\operatorname{LTC}_{f}(U)$ for $f$ a function of special bounded variation and finite level total curvature on $U$. Of course, $\frac{1}{2 \pi} \mathrm{TC}\left(\partial E_{f}(t), U\right)$ will not coincide with $\chi\left(E_{f}(t) \cap U\right)$ when the boundary of the excursion set is not included in the observation window $U$. See also an illustration of this fact on Figure 2. But it can be seen as a "modified" Euler Characteristic, in a sense very similar to the one used in the book of Adler and Taylor [6] or in the paper of Estrade and León [19], where critical points in $U$ are only taken into account, and not the ones on the boundary of $U$. Moreover, when considering large domains (that is $r U$ for $r$ going to infinity) the total curvature (in expectation) will grow like $r^{2} \mathcal{L}(U)$ whereas the sum of the turning angles on $\partial(r U)$ will (in expectation also) grow like $r \mathcal{H}^{1}(\partial U)$, being negligible for large $r$.

We will show how the perimeter and the level total curvature integrals can be computed in different situations and we will apply it for computing Perimeter and Euler Characteristic densities of some stationary fields. The first situation is the case of smooth (at least $C^{2}$ ) functions, and the second situation is the case of sums of piecewise constant functions (also called elementary functions).

\section{Level integrals of smooth functions and random fields}

\subsection{The case of smooth functions}

We start by considering the case of smooth functions. In the sequel, for $f$ a $C^{2}$ function we denote by $\nabla f$ its gradient vector and by $D^{2} f$ its Hessian matrix.

Proposition 1. Let $U$ be an open bounded subset of $\mathbb{R}^{2}$ such that its boundary is a piecewise regular curve. Let $f$ be a $C^{2}$ function defined on an open set containing $\bar{U}$. Then $f$ is of special 


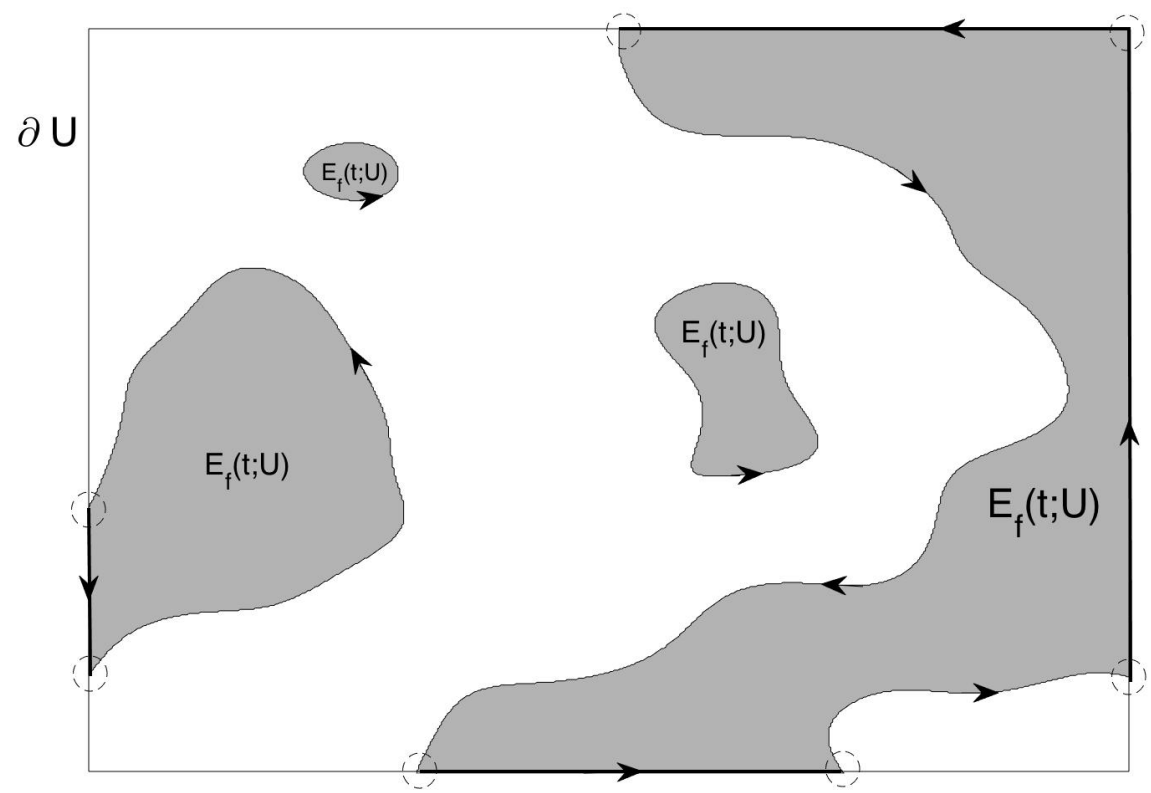

FIG 2. By the Gauss-Bonnet theorem, the Euler Characteristic of the excursion set $E_{f}(t) \cap U$ (in gray) is equal to the total curvature of its boundary in the open rectangular domain $U$ plus the turning angles at the points where $\partial\left(E_{f}(t) \cap U\right)$ meets $\partial U$ and also plus $\pi / 2$ for each of the corners of the rectangle $U$ that are in $E_{f}(t)$. All these special points are the ones marked by the small dashed circles on the figure.

bounded variation and of finite level total curvature integral on $U$ with

$$
V_{f}(U)=\int_{U}\|\nabla f(x)\| d x \quad \text { and } \quad \operatorname{LTaC}_{f}(U) \leq \int_{U}\left\|D^{2} f(x)\right\|_{2} d x
$$

where $\|\cdot\|_{2}$ is the matrix norm subordinated to the Euclidean norm. Moreover, for $h$ a bounded continuous function on $\mathbb{R}$, the level perimeter and total curvature integrals of $f$ are given by

$$
\begin{gathered}
\operatorname{LP}_{f}(h, U)=\int_{U} h(f(x))\|\nabla f(x)\| d x \quad \text { and } \\
\operatorname{LTC}_{f}(h, U)=-\int_{U} h(f(x)) \frac{D^{2} f(x) \cdot\left(\nabla f(x)^{\perp}, \nabla f(x)^{\perp}\right)}{\|\nabla f(x)\|^{2}} \mathbf{1}_{\|\nabla f(x)\|>0} d x,
\end{gathered}
$$

where if $A=\left(a_{i j}\right)_{1 \leq i, j \leq 2}$ is a $2 \times 2$ symmetric matrix and $y=\left(y_{1}, y_{2}\right) \in \mathbb{R}^{2}$, we use the notation

$$
A .(y, y)={ }^{t} y A y=a_{11} y_{1}^{2}+a_{22} y_{2}^{2}+2 a_{12} y_{1} y_{2} .
$$

Proof. Note that since $f$ is $C^{1}$ on an open set containing $\bar{U}$ we have that $f \in S B V(U)$ and thus the set

$$
E_{f}(t):=\{x \in U ; f(x) \geq t\}
$$

is of finite perimeter in $U$ for a.e. $t \in \mathbb{R}$ by the co-area formula (see Theorem 3.40 [7]). Moreover, since $f$ is actually $C^{2}$ on an open set containing $\bar{U}$, by Morse-Sard theorem (see [22] p.69 for instance), the set of critical values of $f$ and of critical values of $f$ restricted to $\partial U$, denoted as 
$f_{/ \partial U}$ has measure 0 in $\mathbb{R}$. Hence for a.e. $t \in \mathbb{R}$, for all points $x \in U$ such that $f(x)=t$ then $\nabla f(x) \neq 0$ and for all $x \in \mathcal{R}_{\partial U}$ such that $f(x)=t$ then $\left\langle\nabla f(x), n_{\partial U}(x)^{\perp}\right\rangle \neq 0$. Let $t$ be such a non-critical value. Let us show that $E_{f}(t)$ is an elementary set. We first notice that

$$
\partial E_{f}(t)=\{x \in U ; f(x)=t\} \cup\{x \in \partial U ; f(x) \geq t\}
$$

By the implicit function theorem, if $x \in \partial E_{f}(t) \cap U$, it is a regular point and one can find a parametrization $\gamma$ given by an implicit form $f(\gamma(s))=t$, with $x=\gamma(s)$, normal vector $\gamma^{\prime}(s)^{\perp}=$ $\nabla f(x) /\|\nabla f(x)\|$ and curvature given by

$$
\kappa_{f}(x):=-\frac{D^{2} f(x) \cdot\left(\nabla f^{\perp}(x), \nabla f^{\perp}(x)\right)}{\|\nabla f(x)\|^{3}} .
$$

From the compactness of $\partial E_{f}(t)$ and the fact that $t$ is not a critical value for both $f$ and $f_{/} \partial U$ we deduce that $\partial E_{f}(t)$ is the finite union of disjoint piecewise $C^{2}$ Jordan curves that have a finite number of corner points (more precisely this number is bounded by $\mathcal{H}^{0}\left(\mathcal{C}_{\partial U}\right)$ plus the number of $x \in \partial U$ such that $f(x)=t$, which is finite). Therefore, $E_{f}(t)$ is an elementary set and we have that

$$
\begin{aligned}
\operatorname{TaC}\left(\partial E_{f}(t), U\right) & =\int_{\partial E_{f}(t) \cap U}\left|\kappa_{f}(x)\right| \mathcal{H}^{1}(d x)<+\infty \\
\text { and } \operatorname{TC}\left(\partial E_{f}(t), U\right) & =\int_{\partial E_{f}(t) \cap U} \kappa_{f}(x) \mathcal{H}^{1}(d x) .
\end{aligned}
$$

Now let us define $\kappa_{f}$ as a measurable function on $U$ by setting for all $x \in U$

$$
\kappa_{f}(x)=-\frac{D^{2} f(x) \cdot\left(\nabla f^{\perp}(x), \nabla f^{\perp}(x)\right)}{\|\nabla f(x)\|^{3}} \mathbf{1}_{\|\nabla f(x)\|>0} .
$$

Let us recall the co-area formula for Lipschitz mappings (see [20] p.117 for instance): for any non-negative measurable or $\mathcal{L}$-integrable function $g$, the function $t \mapsto \int_{\partial E_{f}(t) \cap U} g(x) \mathcal{H}^{1}(d x)$ is measurable and

$$
\int_{U} g(x)\|\nabla f(x)\| d x=\int_{\mathbb{R}} \int_{\partial E_{f}(t) \cap U} g(x) \mathcal{H}^{1}(d x) d t .
$$

Taking $g=1$, we recover the co-area formula:

$$
\int_{U}\|\nabla f(x)\| d x=\int_{\mathbb{R}} \mathcal{H}^{1}\left(\partial E_{f}(t) \cap U\right) d t=V_{f}(U),
$$

while for $g=\left|\kappa_{f}\right|$,

$$
\begin{aligned}
\operatorname{LTaC}_{f}(U)=\int_{\mathbb{R}} \int_{\partial E_{f}(t) \cap U}\left|\kappa_{f}(x)\right| \mathcal{H}^{1}(d x) & =\int_{U}\left|\kappa_{f}(x)\right|\|\nabla f(x)\| d x \\
& \leq \int_{U}\left\|D^{2} f(x)\right\|_{2} d x
\end{aligned}
$$

in view of (4). Therefore $t \mapsto \operatorname{TaC}\left(\partial E_{f}(t), U\right)$ is integrable on $\mathbb{R}$ and $f$ is of finite level total curvature integral on $U$. Moreover, for $h$ a bounded continuous function on $\mathbb{R}$, using again twice the co-area formula but now with $\max (g, 0)$ and $-\min (g, 0)$ for $g=h \circ f$ or $g=(h \circ f) \kappa_{f}$, and 
subtracting we get

$$
\begin{aligned}
\operatorname{LP}_{f}(h, U) & =\int_{U} h(f(x))\|\nabla f(x)\| d x \\
\operatorname{LTC}_{f}(h, U) & =\int_{U} h(f(x)) \kappa_{f}(x)\|\nabla f(x)\| d x \\
& =-\int_{U} h(f(x)) \frac{D^{2} f(x) \cdot\left(\nabla f(x)^{\perp}, \nabla f(x)^{\perp}\right)}{\|\nabla f(x)\|^{2}} \mathbf{1}_{\|\nabla f(x)\|>0} d x .
\end{aligned}
$$

This can clearly be extended to complex valued functions $h$ by linearity.

\subsection{A general result for smooth stationary random fields}

In this section we consider a smooth stationary random field $X$ defined on $\mathbb{R}^{2}$. Let us introduce some notations for the derivatives of $X$. A point $x \in \mathbb{R}^{2}$ is defined by its two coordinates $x=\left(x_{1}, x_{2}\right)$ and we denote for $j, k=1,2$,

$$
X_{j}:=\frac{\partial X}{\partial x_{j}} \quad \text { and } \quad X_{j k}:=\frac{\partial^{2} X}{\partial x_{j} \partial x_{k}} .
$$

With these notations it follows that $\nabla X=\left(\begin{array}{c}X_{1} \\ X_{2}\end{array}\right)$ and $D^{2} X=\left(\begin{array}{cc}X_{11} & X_{12} \\ X_{12} & X_{22}\end{array}\right)$. Note that since $X$ is stationary, for any $x \in \mathbb{R}^{2}$,

$$
\left(X(x), \nabla X(x), D^{2} X(x)\right) \stackrel{d}{=}\left(X(0), \nabla X(0), D^{2} X(0)\right) .
$$

When $X, \nabla X$ and $D^{2} X$ have also finite second order moment, $X(x)$ and $\nabla X(x)$ are not correlated, as well as $\nabla X(x)$ and $D^{2} X(x)$ (see [1] p.31 for instance). This is very useful for Gaussian fields since it implies that $\nabla X(x)$ is independent from $\left(X(x), D^{2} X(x)\right)$.

Using the result of Section 3.1 and the stationarity of $X$, we have the following formula.

Theorem 2. Let $U$ be an open bounded subset of $\mathbb{R}^{2}$ such that its boundary is a piecewise regular curve. Let $X$ be a stationary $C^{2}$ random field on $\mathbb{R}^{2}$, such that $X(0), X_{j}(0)$ and $X_{j k}(0)$ have finite expectations for all $j, k=1,2$. Then, a.s., $X \in S B V(U)$ with $D X=\nabla X \mathcal{L}$ and for a.e. $t \in \mathbb{R}$, the random variables $\operatorname{Per}\left(E_{X}(t), U\right)$ and $\operatorname{TC}\left(\partial E_{X}(t), U\right)$ have finite expectation such that for all $h$ bounded continuous function on $\mathbb{R}$, one has

$$
\begin{aligned}
\mathbb{E}\left(\operatorname{LP}_{X}(h, U)\right)=\int_{\mathbb{R}} h(t) \mathbb{E}\left(\operatorname{Per}\left(E_{X}(t), U\right)\right) d t & =\mathcal{L}(U) \overline{\operatorname{LP}_{X}}(h) \quad \text { and } \\
\mathbb{E}\left(\operatorname{LTC}_{X}(h, U)\right) & =\int_{\mathbb{R}} h(t) \mathbb{E}\left(\mathrm{TC}\left(\partial E_{X}(t), U\right)\right) d t=\mathcal{L}(U) \overline{\operatorname{LTC}_{X}}(h)
\end{aligned}
$$

with

$$
\begin{aligned}
\overline{\mathrm{LP}_{X}}(h) & =\mathbb{E}(h(X(0))\|\nabla X(0)\|) \quad \text { and } \\
\overline{\operatorname{LTC}_{X}}(h) & =-\mathbb{E}\left(h(X(0)) \frac{D^{2} X(0) \cdot\left(\nabla X(0)^{\perp}, \nabla X(0)^{\perp}\right)}{\|\nabla X(0)\|^{2}} \mathbf{1}_{\|\nabla X(0)\|>0}\right) .
\end{aligned}
$$


It follows that when the field $X$ is isotropic, i.e. $X \circ A \stackrel{f d d}{=} X$ for all orthogonal matrices $A$, then the above formulas reduce to, $\forall j=1,2$,

$$
\begin{aligned}
\overline{\operatorname{LP}_{X}}(h) & =\frac{\pi}{2} \mathbb{E}\left(h(X(0))\left|X_{j}(0)\right|\right) \quad \text { and } \\
\overline{\operatorname{LTC}_{X}}(h) & =\overline{\operatorname{LTC}_{X}^{(1)}}(h)+\overline{\operatorname{LTC}_{X}^{(2)}}(h),
\end{aligned}
$$

where

$$
\begin{aligned}
& \overline{\operatorname{LTC}_{X}^{(1)}}(h)=-\mathbb{E}\left(h(X(0)) X_{j j}(0) \mathbf{1}_{\|\nabla X(0)\|>0}\right) \\
& \left.\overline{\operatorname{LTC}_{X}^{(2)}}(h)=4 \mathbb{E}\left(h(X(0)) \frac{X_{1}(0) X_{2}(0)}{\|\nabla X(0)\|^{2}} X_{12}(0) \mathbf{1}_{\|\nabla X(0)\|>0}\right)\right) .
\end{aligned}
$$

It follows that when $X(0)$ admits a probability density $p_{X(0)}$, we get for the densities defined in the introduction, for almost every $t \in \mathbb{R}, \forall j=1,2$,

$$
\begin{aligned}
\overline{\operatorname{Per}\left(E_{X}(t)\right)}= & \frac{\pi}{2} \mathbb{E}\left(\mid X_{j}(0) \| X(0)=t\right) p_{X(0)}(t) \\
\overline{\operatorname{TC}\left(E_{X}(t)\right)}= & {\left[-\mathbb{E}\left(X_{j j}(0) \mathbf{1}_{\|\nabla X(0)\|>0} \mid X(0)=t\right)\right.} \\
& \left.+4 \mathbb{E}\left(\frac{X_{1}(0) X_{2}(0)}{\|\nabla X(0)\|^{2}} X_{12}(0) \mathbf{1}_{\|\nabla X(0)\|>0} \mid X(0)=t\right)\right] p_{X(0)}(t) .
\end{aligned}
$$

Remark: Let us remark that assuming that the field $X$ is isotropic and symmetric, i.e. $X \stackrel{f d d}{=}$ $-X$, we get $\overline{\operatorname{LTC}_{X}}(h)=0$ for any even bounded function $h$ and in particular for $h=1$. If moreover $\left(X(0), X_{1}(0), X_{2}(0), X_{12}(0)\right) \stackrel{d}{=}\left(X(0),-X_{1}(0), X_{2}(0), X_{12}(0)\right)$ we get $\overline{\operatorname{LTC}_{X}^{(2)}}(h)=0$ for any bounded function $h$. This is in particular the case for isotropic fields satisfying $\nabla X(0)$ independent from $\left(X(0), X_{12}(0)\right)$, as centered stationary isotropic Gaussian random fields.

Proof. According to Proposition 1, since $X$ is a.s $C^{2}$, it is a.s. of special bounded variation and of finite level curvature integral on $U$ with

$$
V_{X}(U)=\int_{U}\|\nabla X(x)\| d x \quad \text { and } \quad \operatorname{LTaC}_{X}(U) \leq \int_{U}\left\|D^{2} X(x)\right\|_{2} d x \quad \text { a.s.. }
$$

Since $X$ is stationary, the finite expectation assumption implies that $V_{X}(U)$ and $\operatorname{LTaC}_{X}(U)$ are non-negative random variables with finite expectation. By Fubini's Theorem, this implies that $(t, \omega) \mapsto \operatorname{Per}\left(E_{X(\omega)}(t), U\right) \in L^{1}(\mathbb{R} \times \Omega)$ and $(t, \omega) \mapsto \operatorname{TaC}\left(\partial E_{X(\omega)}(t), U\right) \in L^{1}(\mathbb{R} \times \Omega)$ so that we also have $(t, \omega) \mapsto \operatorname{TC}\left(\partial E_{X(\omega)}(t), U\right) \in L^{1}(\mathbb{R} \times \Omega)$. Moreover, a.s., for any $h$ bounded continuous function on $\mathbb{R}$,

$$
\operatorname{LP}_{X}(h, U)=\int_{\mathbb{R}} h(t) \operatorname{Per}\left(E_{X}(t), U\right) d t \quad \text { and } \quad \operatorname{LTC}_{X}(h, U)=\int_{\mathbb{R}} h(t) \operatorname{TC}\left(\partial E_{X}(t), U\right) d t,
$$

with

$$
\begin{gathered}
\operatorname{LP}_{X}(h, U)=\int_{U} h(X(x))\|\nabla X(x)\| d x \text { and } \\
\operatorname{LTC}_{X}(h, U)=-\int_{U} h(X(x)) \frac{D^{2} X(x) \cdot\left(\nabla X(x)^{\perp}, \nabla X(x)^{\perp}\right)}{\|\nabla X(x)\|^{2}} \mathbf{1}_{\|\nabla X(x)\|>0} d x .
\end{gathered}
$$

Hence, taking the expectation, Fubini's Theorem and the stationarity of $X$ imply the results. 
Under the assumption that the field is isotropic, we can exploit further on this formula. First let us recall that by Taylor formula, since $X$ is a.s. $C^{2}$ we have a.s. for all $x, z \in \mathbb{R}^{2}$,

$$
X(x+z)=X(x)+\langle\nabla X(x), z\rangle+\frac{1}{2} D^{2} X(x) \cdot(z, z)+o_{\|z\| \rightarrow 0}\left(\|z\|^{2}\right) .
$$

In particular we obtain that, for any orthogonal matrix $A$,

$$
\nabla(X \circ A)(x)={ }^{t} A \nabla X(A x) \text { and } D^{2}(X \circ A)(x)={ }^{t} A\left(D^{2} X\right)(A x) A .
$$

Since $X \circ A \stackrel{f d d}{=} X$, we deduce that

$$
\left(X(x), \nabla X(x), D^{2} X(x)\right) \stackrel{d}{=}\left(X(A x),{ }^{t} A \nabla X(A x),{ }^{t} A\left(D^{2} X\right)(A x) A\right),
$$

and specifying to $x=0$, it follows that

$$
\left(X(0), \nabla X(0), D^{2} X(0)\right) \stackrel{d}{=}\left(X(0),{ }^{t} A \nabla X(0),{ }^{t} A\left(D^{2} X\right)(0) A\right) .
$$

Hence $(X(0), \nabla X(0)) \stackrel{d}{=}\left(X(0),{ }^{t} A \nabla X(0)\right)$ and for any $\theta \in[0,2 \pi)$, denoting $u(\theta)=(\cos \theta, \sin \theta) \in$ $S^{1}$, one has $\left(X(0), X_{j}(0)\right) \stackrel{d}{=}(X(0),\langle u(\theta), \nabla X(0)\rangle)$, according to orthogonal invariance (see Proposition 4.8 of [12]). Moreover $\|\nabla X(0)\|=\frac{1}{4} \int_{0}^{2 \pi}|\langle u(\theta), \nabla X(0)\rangle| d \theta$ so that we deduce

$$
\mathbb{E}(h(X(0))\|\nabla X(0)\|)=\frac{1}{4} \int_{0}^{2 \pi} \mathbb{E}(h(X(0))|\langle u(\theta), \nabla X(0)\rangle|) d \theta=\frac{\pi}{2} \mathbb{E}\left(h(X(0))\left|X_{j}(0)\right|\right),
$$

and the result for $\overline{\mathrm{LP}_{X}}(h)$ follows.

Now, let us consider $\overline{\operatorname{LTC}_{X}}(h)$. We introduce the random variable $\Theta$ with values in $2 \pi \mathbb{T}$ (identified with $[0,2 \pi)$ ), such that when $\|\nabla X(0)\|>0$,

$$
\nabla X(0)=\left(\begin{array}{c}
X_{1}(0) \\
X_{2}(0)
\end{array}\right)=\|\nabla X(0)\|\left(\begin{array}{c}
\cos \Theta \\
\sin \Theta
\end{array}\right)
$$

For the sake of brevity, we drop the point notation (0) in the sequel of the proof. Then

$$
\begin{aligned}
& \mathbb{E}\left(h(X) \frac{D^{2} X \cdot\left(\nabla X^{\perp}, \nabla X^{\perp}\right)}{\|\nabla X\|^{2}} \mathbf{1}_{\|\nabla X\|>0}\right) \\
= & \mathbb{E}\left(h(X)\left(X_{11} \sin ^{2} \Theta+X_{22} \cos ^{2} \Theta-2 X_{12} \sin \Theta \cos \Theta\right) \mathbf{1}_{\|\nabla X\|>0}\right) \\
= & \mathbb{E}\left(h(X)\left(\frac{X_{11}+X_{22}}{2}+\frac{X_{22}-X_{11}+2 i X_{12}}{4} e^{2 i \Theta}+\frac{X_{22}-X_{11}-2 i X_{12}}{4} e^{-2 i \Theta}\right) \mathbf{1}_{\|\nabla X\|>0}\right) .
\end{aligned}
$$

Hence introducing the complex random variables $J=\|\nabla X\| e^{i \Theta} \mathbf{1}_{\|\nabla X\|>0}$ and $K=\frac{1}{4}\left(X_{22}-X_{11}-\right.$ $\left.2 i X_{12}\right)$, the rotation invariance (5) implies that for any $\theta \in[0,2 \pi)$,

$$
(X, J, K) \stackrel{d}{=}\left(X, e^{i \theta} J, e^{2 i \theta} K\right) .
$$

Now, we remark that

$$
\mathbb{E}\left(h(X) \frac{D^{2} X \cdot\left(\nabla X^{\perp}, \nabla X^{\perp}\right)}{\|\nabla X\|^{2}} \mathbf{1}_{\|\nabla X\|>0}\right)=\alpha_{0}(h)+\alpha_{2}(h)+\overline{\alpha_{2}(\bar{h})},
$$


where

$$
\alpha_{0}(h)=\mathbb{E}\left(h(X)\left(\frac{X_{11}+X_{22}}{2}\right) \mathbf{1}_{\|\nabla X\|>0}\right)=\mathbb{E}\left(h(X) X_{j j} \mathbf{1}_{\|\nabla X\|>0}\right)=-\overline{\operatorname{LTC}_{X}^{(1)}}(h),
$$

for any $j \in\{1,2\}$, using the fact that $\left(X, X_{11}\right) \stackrel{d}{=}\left(X, X_{22}\right)$ by $(5)$, and

$$
\alpha_{2}(h)=\mathbb{E}\left(h(X) \bar{K} e^{2 i \Theta} \mathbf{1}_{\|\nabla X\|>0}\right) .
$$

But (6) implies that for all $n \in \mathbb{Z}$ with $n \neq 2$ and $\theta \in[0,2 \pi)$, we have $\mathbb{E}\left(h(X) \bar{K} e^{i n \Theta} \mathbf{1}_{\|\nabla X\|>0}\right)=$ $e^{i(n-2) \theta} \mathbb{E}\left(h(X) \bar{K} e^{n i \Theta} \mathbf{1}_{\|\nabla X\|>0}\right)=0$. It follows that

$$
\alpha_{2}(h)=\mathbb{E}\left(h(X) \bar{K}\left(e^{2 i \Theta}-e^{-2 i \Theta}\right) \mathbf{1}_{\|\nabla X\|>0}\right)=2 i \mathbb{E}\left(h(X) \bar{K} \sin (2 \Theta) \mathbf{1}_{\|\nabla X\|>0}\right) .
$$

Finally we deduce that when $h$ is real-valued, denoting by $\Re$ the real part of a complex number, since $\Re(i \bar{K})=-\frac{1}{2} X_{12}$,

$$
2 \Re \alpha_{2}(h)=-2 \mathbb{E}\left(h(X) X_{12} \sin (2 \Theta) \mathbf{1}_{\|\nabla X\|>0}\right)=-\overline{\operatorname{LTC}_{X}^{(2)}}(h) .
$$

This concludes the proof for real-valued functions $h$. The result clearly extends to complex-valued functions by linearity. Moreover, when $X(0)$ admits a density $p_{X(0)}$, we can further write

$$
\begin{aligned}
\overline{\operatorname{LP}_{X}}(h) & =\int_{\mathbb{R}} h(t) \frac{\pi}{2} \mathbb{E}\left(\mid X_{j}(0) \| X(0)=t\right) p_{X(0)}(t) d t \\
\overline{\operatorname{LTC}_{X}^{(1)}}(h) & =-\int_{\mathbb{R}} h(t) \mathbb{E}\left(X_{j j}(0) \mathbf{1}_{\|\nabla X(0)\|>0} \mid X(0)=t\right) p_{X(0)}(t) d t \\
\overline{\operatorname{LTC}_{X}^{(2)}}(h) & =4 \int_{\mathbb{R}} h(t) \mathbb{E}\left(\frac{X_{1}(0) X_{2}(0)}{\|\nabla X(0)\|^{2}} X_{12}(0) \mathbf{1}_{\|\nabla X(0)\|>0} \mid X(0)=t\right) p_{X(0)}(t) d t
\end{aligned}
$$

such that for any continuous bounded function $h$,

$$
\begin{aligned}
\int_{\mathbb{R}} h(t) \overline{\operatorname{Per}\left(E_{X}(t)\right)} d t & =\int_{\mathbb{R}} h(t) \frac{\pi}{2} \mathbb{E}\left(\mid X_{j}(0) \| X(0)=t\right) p_{X(0)}(t) d t \quad \text { and } \\
\int_{\mathbb{R}} h(t) \overline{\operatorname{TC}\left(E_{X}(t)\right)} d t & =\int_{\mathbb{R}} h(t)\left[-\mathbb{E}\left(X_{j j}(0) \mathbf{1}_{\|\nabla X(0)\|>0} \mid X(0)=t\right)\right. \\
& \left.+4 \mathbb{E}\left(\frac{X_{1}(0) X_{2}(0)}{\|\nabla X(0)\|^{2}} X_{12}(0) \mathbf{1}_{\|\nabla X(0)\|>0} \mid X(0)=t\right)\right] p_{X(0)}(t) d t
\end{aligned}
$$

implying the stated equalities for almost every $t \in \mathbb{R}$.

Example: Let $X$ be a stationary centered $C^{2}$ isotropic Gaussian random field. Then, $X_{1}(0)$ being independent from $X(0)$, we get $\mathbb{E}\left(\left|X_{1}(0)\right| \mid X(0)\right)=\mathbb{E}\left(\left|X_{1}(0)\right|\right)=\sqrt{\frac{2 \lambda_{2}}{\pi}}$, where $\lambda_{2}=$ $\operatorname{Var}\left(X_{1}(0)\right)>0$ denotes the second spectral moment. Moreover,

$$
\mathbb{E}\left(X_{11}(0) \mid X(0)\right)=\frac{\operatorname{Cov}\left(X(0), X_{11}(0)\right)}{\operatorname{Var}(X(0))} X(0)=\frac{\partial_{1}^{2} \rho_{X}(0)}{\rho_{X}(0)} X(0)=\frac{-\lambda_{2}}{\sigma^{2}} X(0),
$$

where $\rho_{X}(x)=\operatorname{Cov}(X(x), X(0))$ and $\sigma^{2}=\rho_{X}(0)$, while by independence

$$
\mathbb{E}\left(\frac{X_{1}(0) X_{2}(0)}{\|\nabla X(0)\|^{2}} X_{12}(0) \mid X(0)\right)=\mathbb{E}\left(\frac{X_{1}(0) X_{2}(0)}{\|\nabla X(0)\|^{2}}\right) \mathbb{E}\left(X_{12}(0) \mid X(0)\right)=0 .
$$


Hence, since $\mathbb{P}(\|\nabla X(0)\|=0)=0$, we get in this case that, for almost every $t \in \mathbb{R}$,

$$
\begin{aligned}
\overline{\operatorname{Per}\left(E_{X}(t)\right)} & =\sqrt{\frac{\pi \lambda_{2}}{2}} \frac{1}{\sigma \sqrt{2 \pi}} e^{-\frac{t^{2}}{2 \sigma^{2}}}, \\
\overline{\operatorname{TC}\left(E_{X}(t)\right)} & =\frac{\lambda_{2}}{\sigma^{2}} t \frac{1}{\sigma \sqrt{2 \pi}} e^{-\frac{t^{2}}{2 \sigma^{2}}} .
\end{aligned}
$$

Let us emphasize that this last expression yields exactly to the formula obtained for $\left.2 \pi \mathbb{E}\left(\chi\left(E_{X}(t) \cap \bar{U}\right)\right)\right)$, stated for all $t \in \mathbb{R}$, under additional assumptions on $X$ (see (3.2.8) of [2] for instance), where $\chi$ denotes the DT (Differential Topology) Characteristic of the set and therefore $\overline{\mathrm{TC}\left(E_{X}(t)\right)} / 2 \pi$ corresponds to the Euler characteristic density of $E_{X}(t)$.

Examples of such stationary isotropic random fields with comparisons between the theoretical values of $\overline{\mathrm{TC}\left(E_{X}(t)\right)} / 2 \pi$ and an empirical estimate of Euler Characteristic on the square of fixed size $[0,1]$ are shown on Figures 3 and 4 (with $\sigma^{2}=1$ and $\lambda_{2}=2 T^{2}$ for $T=10$ and $T=100$ ). The captions of the figures give the practical and technical details of the simulations. Note that in view of the covariance functions, a scaling relation may be set between $T$ and the size of the square, explaining the convergence without boundary effects as $T$ increases.

It is more difficult to compute exact formulas in the general case where the gradient $\nabla X(0)$ is not independent from $\left(X(0), D^{2} X(0)\right)$. However, we can use the following expressions that allow more tractable computations. The proofs are technical and postponed to Appendix (Section 5).

Proposition 2. Let $W, X_{1}, X_{2}$ be real random variables and let $\varepsilon \in(0,1]$.

1. If $\mathbb{E}\left(\left|W X_{1}\right|\right)<+\infty$ and $\mathbb{E}\left(|W|\left|X_{1}\right|^{1+\varepsilon}\right)<+\infty$, then

$$
\mathbb{E}\left(W\left|X_{1}\right|\right)=\frac{2}{\pi} \int_{0}^{+\infty} \frac{1}{u} \mathbb{E}\left(W X_{1} \sin \left(u X_{1}\right)\right) d u .
$$

2. If $\mathbb{E}(|W|)<+\infty$ and $\mathbb{E}\left(|W|\left|X_{1} X_{2}\right|^{\varepsilon}\right)<+\infty$, then

$$
\mathbb{E}\left(W \frac{X_{1} X_{2}}{X_{1}^{2}+X_{2}^{2}} \mathbf{1}_{X_{1}^{2}+X_{2}^{2}>0}\right)=\frac{4}{\pi} \int_{0}^{+\infty} \int_{0}^{+\infty} \frac{v_{1} v_{2}}{\left(v_{1}^{2}+v_{2}^{2}\right)^{2}} \mathbb{E}\left(W \sin \left(v_{1} X_{1}\right) \sin \left(v_{2} X_{2}\right)\right) d v_{1} d v_{2} .
$$

Here, the improper integrals $\int_{0}^{+\infty}$ are obtained as $\lim _{M \rightarrow+\infty} \int_{0}^{M}$.

Another useful result can be stated using the invariance property (6). The proof of this result is also postponed to the Appendix (Section 5).

Proposition 3. Let $W, J, K$ be complex random variables such that for any $\theta \in[0,2 \pi)$,

$$
(W, J, K) \stackrel{d}{=}\left(W, e^{i \theta} J, e^{2 i \theta} K\right)
$$

and $\mathbb{E}(|W K|)<+\infty$. Then, writing $J=R e^{i \Theta} \mathbf{1}_{|J|>0}$, on the one hand, for any $g: 2 \pi \mathbb{T} \rightarrow \mathbb{R}$ continuous bounded $2 \pi$ periodic function, one has

$$
\mathbb{E}\left(W \bar{K} g(\Theta) \mathbf{1}_{|J|>0}\right)=c_{2}(g) \mathbb{E}\left(W \bar{K} e^{2 i \Theta} \mathbf{1}_{|J|>0}\right),
$$

with $c_{2}(g)=\frac{1}{2 \pi} \int_{0}^{2 \pi} e^{-2 i \pi \theta} g(\theta) d \theta$. On the other hand, if there exists $\varepsilon>0$ such that $\mathbb{E}\left(|W K||J|^{\varepsilon}\right)<$ $+\infty$, then

$$
\mathbb{E}\left(W \bar{K} \sin (2 \Theta) \mathbf{1}_{|J|>0}\right)=i \int_{0}^{+\infty} \frac{1}{v} \mathbb{E}(W \bar{K} \cos (v \Re(J))) d v .
$$



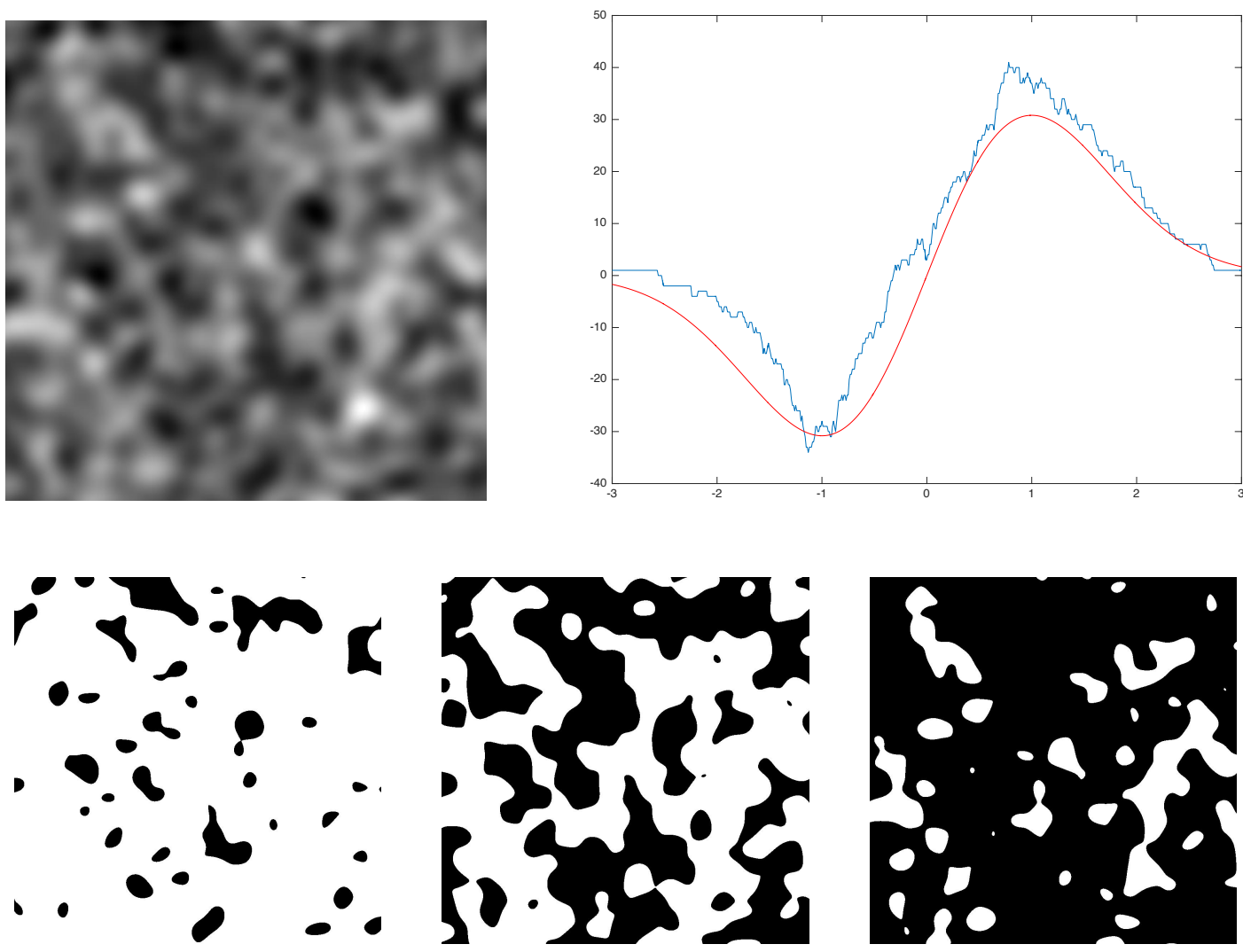

FIG 3. Gaussian random field with covariance $\rho(x)=e^{-T^{2}\|x\|^{2}}$ for $T=10$. This sample has been obtained using Matlab, with a discretized domain of size $2^{10} \times 2^{10}$ pixels, using the circulant embedding matrix method. Top right figure: empirical Euler Characteristic as a function of the level $t$ (computed thanks to the Matlab function bweuler), compared with the theoretical value (red curve) of Equation (9). Bottom line: Three excursion sets corresponding respectively from left to right to the level $t=-1, t=0$ (that is the "critical level" where the Euler Characteristic turns from negative to positive), and $t=1$.

Remark: A closely related result is given in Corollary 2.3 of [23], that should rewrite in our stationary setting, under additional assumption on $X$, as

$\int_{\mathbb{R}} h(t) \overline{\chi\left(E_{X}(t)\right)} d t=-\mathbb{E}\left(h(X(0))\left[\sum_{j=1}^{2} \mathbf{I}_{\nabla X(0) \in Q_{j}} X_{j j}(0)\right]+h^{\prime}(X(0))\left[\sum_{j=1}^{2} \mathbf{I}_{\nabla X(0) \in Q_{j}} X_{j}(0)^{2}\right]\right)$,

for $h: \mathbb{R} \rightarrow \mathbb{R}$ a $C^{1}$ function with compact support, $Q_{1}=\left\{x=\left(x_{1}, x_{2}\right) ; x_{2}<x_{1}<0\right\}$ and $Q_{2}=\left\{x=\left(x_{1}, x_{2}\right) ; x_{1}<x_{2}<0\right\}$, and where $\overline{\chi\left(E_{X}(t)\right)}$ stands for an Euler Characteristic density. Under the assumption that $X$ is also isotropic, according to Remark 2.5 of [23],

$$
\mathbb{E}\left(h^{\prime}(X(0)) \mathbb{I}_{\nabla X(0) \in Q_{j}} X_{j}(0)^{2}\right)=\frac{\pi-2}{16 \pi} \mathbb{E}\left(h^{\prime}(X(0))\|\nabla X(0)\|^{2}\right) .
$$



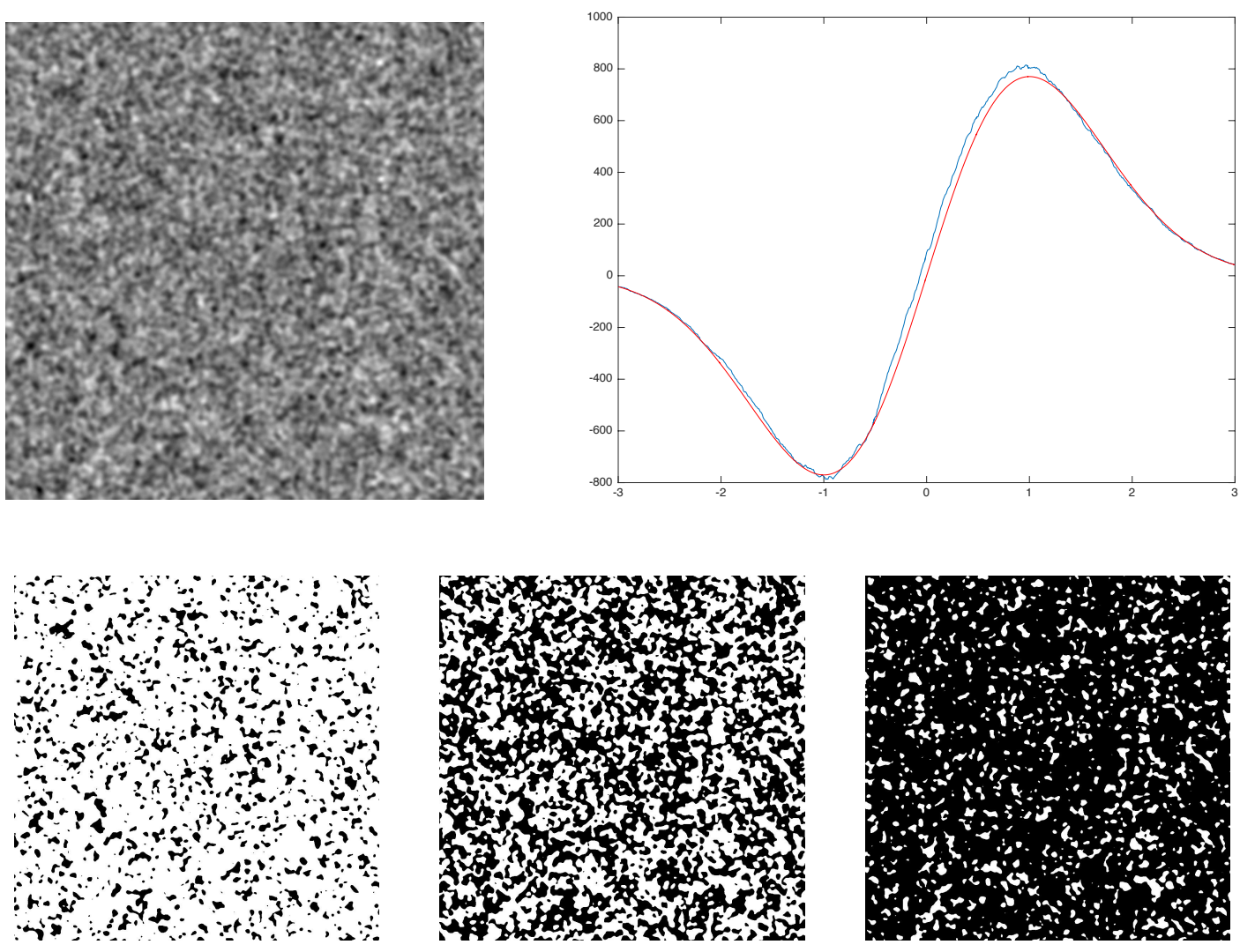

FIG 4. Gaussian random field with covariance $\rho(x)=e^{-T^{2}\|x\|^{2}}$ for $T=100$. This sample has been obtained using Matlab, with a discretized domain of size $2^{10} \times 2^{10}$ pixels, using the circulant embedding matrix method. Top right figure: empirical Euler Characteristic as a function of the level $t$ (computed thanks to the Matlab function bweuler), compared with the theoretical value (red curve) of Equation (9). Bottom line: Three excursion sets corresponding respectively from left to right to the level $t=-1, t=0$ (that is the "critical level" where the Euler Characteristic turns from negative to positive), and $t=1$.

Note that by stationarity, denoting $e_{1}=(1,0)$ and $e_{2}=(0,1)$, for any $j=1,2$,

$$
\begin{aligned}
\mathbb{E}\left(h^{\prime}(X(0)) X_{j}(0)^{2}\right) & =\int_{0}^{1} \mathbb{E}\left(h^{\prime}\left(X\left(t e_{j}\right)\right) X_{j}\left(t e_{j}\right)^{2}\right) d t=\mathbb{E}\left(\int_{0}^{1} h^{\prime}\left(X\left(t e_{j}\right)\right) X_{j}\left(t e_{j}\right)^{2} d t\right) \\
& =-\mathbb{E}\left(\int_{0}^{1} h\left(X\left(t e_{j}\right)\right) X_{j j}\left(t e_{j}\right) d t\right)=-\mathbb{E}\left(h(X(0)) X_{j j}(0)\right),
\end{aligned}
$$

integrating by parts and using again the stationarity of $X$. Moreover,

$$
\mathbb{E}\left(h(X(0)) \mathbb{I}_{\nabla X(0) \in Q_{j}} X_{j j}(0)\right)=\mathbb{E}\left(h(X(0)) g_{j}(\Theta) X_{j j}(0)\right),
$$

with $g_{j}(\theta)=\mathbf{I}_{\theta \in \pi+\frac{\pi}{4}(2-j, 3-j)}$ that is bounded but not continuous. However, if we assume that $\left(X(0), \nabla X(0), X_{j j}(0)\right)$ admits a density we can adapt the arguments of Proposition 3 , to compute $\alpha_{2}(h)=\frac{1}{c_{2}\left(g_{j}\right)} \mathbb{E}\left(h(X(0)) g_{j}(\Theta) \bar{K}\right)$, with $c_{2}\left(g_{1}\right)=-\frac{1}{4 \pi}(1+i)$ and $c_{2}\left(g_{2}\right)=-\frac{1}{4 \pi}(-1+i)$ and we obtain

$$
\left.\mathbb{E}\left(\int_{\mathbb{R}} h(t) \overline{\chi\left(E_{X}(t)\right)}\right) d t\right)=\frac{1}{2 \pi} \int_{\mathbb{R}} h(t) \overline{\operatorname{TC}\left(\partial E_{X}(t)\right)} d t,
$$


by Theorem 2 , since it is also assumed that $\|\nabla X(0)\|>0$ a.s..

\subsection{Smooth shot noise random fields}

We consider here a shot-noise random field defined on $\mathbb{R}^{2}$ by

$$
\forall x \in \mathbb{R}^{2}, \quad X_{\Phi}(x)=\sum_{i \in I} g_{m_{i}}\left(x-x_{i}\right),
$$

where $\Phi=\left\{\left(x_{i}, m_{i}\right)\right\}_{i \in I}$ is a Poisson point process on $\mathbb{R}^{2} \times \mathbb{R}^{d}$, defined on a probability space $(\Omega, \mathcal{A}, \mathbb{P})$, of intensity $\lambda \mathcal{L} \times F$, with $\lambda>0$ real, $\mathcal{L}$ the Lebesgue measure on $\mathbb{R}^{2}$ and $F$ a probability measure on $\mathbb{R}^{d}$. Note that equivalently, we may define $\Phi$ as an independently marked Poisson point process where $\left\{x_{i}\right\}_{i}$ is an homogeneous Poisson point process of intensity $\lambda$ and the $m_{i}$ are "marks", following a law $F(d m)$ on $\mathbb{R}^{d}$ (with $d \geq 1$ ) and independent of the Poisson point process $\left\{x_{i}\right\}_{i}$. Let $g: \mathbb{R}^{2} \times \mathbb{R}^{d} \rightarrow \mathbb{R}$ be a measurable function such that the functions $g_{m}:=g(\cdot, m)$ satisfy

$$
\int_{\mathbb{R}^{2} \times \mathbb{R}^{d}}\left|g_{m}(x)\right| d x F(d m)<+\infty .
$$

Then, the random field $X_{\Phi}$ is well defined as an almost surely locally integrable function on $\mathbb{R}^{2}$ (see [11]). Moreover, the random field $X_{\Phi}$ is stationary. We will first give sufficient conditions to ensure smoothness properties and also isotropy, in order to obtain explicit formulas. But then, since smooth shot noise random fields do not always admit a probability density (we have discussed this through several examples in our first paper [10]), we will have to work with their characteristic functions.

Theorem 3. Assume that $g: \mathbb{R}^{2} \times \mathbb{R}^{d} \rightarrow \mathbb{R}$ is a measurable function such that for $F$-almost every $m \in \mathbb{R}^{d}$ the functions $g_{m}:=g(\cdot, m)$ are $C^{3}$ on $\mathbb{R}^{2}$ satisfying

$$
\int_{\mathbb{R}^{2} \times \mathbb{R}^{d}}\left|D^{\mathbf{j}} g_{m}(x)\right| d x F(d m)<+\infty,
$$

for all $\mathbf{j}=\left(j_{1}, j_{2}\right) \in \mathbb{N}^{2}$ with $|\mathbf{j}|=j_{1}+j_{2} \leq 3$ and where $D^{\mathbf{j}} g_{m}=\frac{\partial^{|\mathbf{j}|} g_{m}}{\partial x_{1}^{j_{1}} \partial x_{2}^{j_{2}}}$. Then $X_{\Phi}$ is a.s. a stationary $C^{2}$ field such that $X, X_{j}, X_{j k}$ have finite expectation for $j, k=1,2$, ensuring the assumptions of Theorem 2.

If we assume moreover that for $F$-a.e. $m$, the function $g_{m}$ is invariant under rotations, then $X_{\Phi}$ is isotropic. It follows that, when we also have

$$
\int_{\mathbb{R}^{2} \times \mathbb{R}^{d}}\left|D^{\mathbf{j}} g_{m}(x)\right|^{2} d x F(d m)<+\infty,
$$

for $|\mathbf{j}| \in\{1,2\}$, then for all $u \in \mathbb{R}$, denoting $h_{u}$ the function $t \mapsto e^{i u t}$,

$$
\begin{aligned}
\overline{\operatorname{LP}_{X_{\Phi}}}\left(h_{u}\right) & =\int_{0}^{+\infty} \frac{1}{v} \varphi(u, v) S_{0}(u, v) d v \\
\overline{\operatorname{LTC}_{X_{\Phi}}^{(1)}}\left(h_{u}\right) & =\varphi(u, 0) S_{1}(u) \\
\overline{\operatorname{LTC}_{X_{\Phi}}^{(2)}}\left(h_{u}\right) & =\int_{0}^{+\infty} \frac{1}{v} \varphi(u, v) S_{2}(u, v) d v
\end{aligned}
$$


where $\varphi$ is the characteristic function of $\left(X_{\Phi}(0), \partial_{1} X_{\Phi}(0)\right)$ given by

$$
\begin{gathered}
\varphi(u, v)=\mathbb{E}\left(e^{i u X_{\Phi}(0)+i v \partial_{1} X_{\Phi}(0)}\right)=\exp \left(\lambda \int_{\mathbb{R}^{d} \times \mathbb{R}^{2}}\left[e^{i\left[u g_{m}(x)+v \partial_{1} g_{m}(x)\right]}-1\right] F(d m) d x\right) \\
S_{0}(u, v)=-i \lambda \int_{\mathbb{R}^{d}} \int_{\mathbb{R}^{2}} \partial_{1} g_{m}(x) e^{i\left[u g_{m}(x)+v \partial_{1} g_{m}(x)\right]} d x F(d m), \\
S_{1}(u)=-\lambda \int_{\mathbb{R}^{d}} \int_{\mathbb{R}^{2}} \partial_{1}^{2} g_{m}(x) e^{i u g_{m}(x)} d x F(d m), \\
S_{2}(u, v)=\lambda \int_{\mathbb{R}^{d}} \int_{\mathbb{R}^{2}}\left[\partial_{2}^{2} g_{m}(x)-\partial_{1}^{2} g_{m}(x)\right] e^{i\left[u g_{m}(x)+v \partial_{1} g_{m}(x)\right]} d x F(d m)
\end{gathered}
$$

and the notation $\partial_{j}$, respectively $\partial_{j}^{2}$, stands for $\frac{\partial}{\partial x_{j}}$, respectively $\frac{\partial^{2}}{\partial x_{j}^{2}}$ for $j \in\{1,2\}$.

Remark: Let us remark that these formulas allow to get an explicit expression for a.e. $t$ as soon as one can identify the right members with some Fourier transforms (with respect to $u$ ).

Proof. Following similar arguments as in Proposition 3 of [10], Condition (12) will ensure that $X_{\Phi}$ is a.s. a stationary $C^{2}$ field such that $X, X_{j}, X_{j k}$ have finite expectation for $j, k=1,2$, and we can differentiate under the sum. In particular, for all $j=1,2$,

$$
\partial_{j} X_{\Phi}(x)=\sum_{i \in I} \partial_{j} g_{m_{i}}\left(x-x_{i}\right) \quad \text { and } \quad \partial_{j}^{2} X_{\Phi}(x)=\sum_{i \in I} \partial_{j}^{2} g_{m_{i}}\left(x-x_{i}\right) .
$$

Hence the general formula of Theorem 2 is valid for $X_{\Phi}$.

Under the additional assumption on the kernel, we can prove isotropy. Actually, for any $k \geq 1$, $u_{1}, \cdots, u_{k} \in \mathbb{R}$ and $y_{1}, \cdots, y_{k} \in \mathbb{R}^{2}$, one has (see for instance [10])

$$
\mathbb{E}\left(e^{i \sum_{j=1}^{k} u_{j} X_{\Phi}\left(y_{j}\right)}\right)=\exp \left(\lambda \int_{\mathbb{R}^{d}} \int_{\mathbb{R}^{2}}\left(e^{i \sum_{j=1}^{k} u_{j} g_{m}\left(y_{j}-x\right)}-1\right) d x F(d m)\right) .
$$

Hence, for any orthogonal matrix $A$, by the change of variables $x=A y$,

$$
\begin{aligned}
\mathbb{E}\left(e^{i \sum_{j=1}^{k} u_{j} X_{\Phi}\left(A y_{j}\right)}\right) & =\exp \left(\lambda \int_{\mathbb{R}^{d}} \int_{\mathbb{R}^{2}}\left(e^{i \sum_{j=1}^{k} u_{j} g_{m}\left(A\left(y_{j}-y\right)\right)}-1\right) d y F(d m)\right) \\
& =\mathbb{E}\left(e^{i \sum_{j=1}^{k} u_{j} X_{\Phi}\left(y_{j}\right)}\right)
\end{aligned}
$$

since $g_{m} \circ A=g_{m}$ for $F$-a.e. $m \in \mathbb{R}^{d}$.

It follows that by Theorem 2 , for any $h$ bounded continuous function on $\mathbb{R}$, one has

$$
\begin{gathered}
\overline{\operatorname{LP}_{X_{\Phi}}}(h)=\frac{\pi}{2} \mathbb{E}\left(h\left(X_{\Phi}(0)\right)\left|\partial_{1} X_{\Phi}(0)\right|\right), \\
\overline{\operatorname{LTC}_{X_{\Phi}}^{(1)}}(h)=-\mathbb{E}\left(h\left(X_{\Phi}(0)\right) \partial_{1}^{2} X_{\Phi}(0) \mathbf{1}_{\left\|\nabla X_{\Phi}(0)\right\|>0}\right) .
\end{gathered}
$$

and for $h$ with real values,

$$
\overline{\operatorname{LTC}_{X_{\Phi}}^{(2)}}(h)=-2 \Re\left(\alpha_{2}(h)\right),
$$

where

$$
\alpha_{2}(h)=\mathbb{E}\left(h\left(X_{\Phi}(0)\right) \overline{K_{\Phi}} e^{2 i \Theta_{\Phi}} \mathbf{1}_{\left\|\nabla X_{\Phi}(0)\right\|>0}\right),
$$


for $K_{\Phi}=\frac{1}{4}\left(\partial_{2}^{2} X_{\Phi}(0)-\partial_{1}^{2} X_{\Phi}(0)-2 i \partial_{12}^{2} X_{\Phi}(0)\right)$ and $\Theta_{\Phi}$ the angle of $\nabla X_{\Phi}(0)$.

Assuming moreover (13) is enough to use Propositions 2 and 3 with $\varepsilon=1$ and we can further write

$$
\begin{aligned}
\alpha_{2}(h) & =2 i \mathbb{E}\left(h\left(X_{\Phi}(0)\right) \overline{K_{\Phi}} \sin \left(2 \Theta_{\Phi}\right) \mathbf{1}_{\left\|\nabla X_{\Phi}(0)\right\|>0}\right) \\
& =-2 \int_{0}^{+\infty} \frac{1}{v} \mathbb{E}\left(h\left(X_{\Phi}(0)\right) \overline{K_{\Phi}} \cos \left(v \partial_{1} X_{\Phi}(0)\right)\right) d v .
\end{aligned}
$$

and

$$
2 \Re \alpha_{2}(h)=-4 \int_{0}^{+\infty} \frac{1}{v} \mathbb{E}\left(h\left(X_{\Phi}(0)\right) \Re \overline{K_{\Phi}} \cos \left(v \partial_{1} X_{\Phi}(0)\right)\right) d v,
$$

so that

$$
\overline{\operatorname{LTC}_{X_{\Phi}}^{(2)}}(h)=\int_{0}^{+\infty} \frac{1}{v} \mathbb{E}\left(h\left(X_{\Phi}(0)\right)\left[\partial_{2}^{2} X_{\Phi}(0)-\partial_{1}^{2} X_{\Phi}(0)\right] \cos \left(v \partial_{1} X_{\Phi}(0)\right)\right) d v,
$$

that may be extended to complex-valued functions $h$. Hence, taking $h_{u}=e^{i u}$ for $u \in \mathbb{R}$ we remark that since $\varphi(u, v)=\varphi(u,-v)=\mathbb{E}\left(h_{u}\left(X_{\Phi}(0)\right) \cos \left(v \partial_{1} X_{\Phi}(0)\right)\right)$,

$$
\mathbb{E}\left(h_{u}\left(X_{\Phi}(0)\right) \partial_{1} X_{\Phi}(0) \sin \left(v \partial_{1} X_{\Phi}(0)\right)\right)=-\frac{\partial \varphi}{\partial v}(u, v)=S_{0}(u, v) \varphi(u, v) .
$$

This leads to the formula for $\overline{\mathrm{LP}_{X_{\Phi}}}\left(h_{u}\right)$. Similarly, using the fact that

$$
\tilde{\varphi}(u, v):=\mathbb{E}\left(e^{i u X_{\Phi}(0)+i v \partial_{1}^{2} X_{\Phi}(0)}\right)=\exp \left(\lambda \int_{\mathbb{R}^{d} \times \mathbb{R}^{2}}\left[e^{i\left[u g_{m}(x)+v \partial_{1}^{2} g_{m}(x)\right]}-1\right] F(d m) d x\right),
$$

we can compute

$$
\overline{\operatorname{LTC}_{X_{\Phi}}^{(1)}}\left(h_{u}\right)=i \frac{\partial \tilde{\varphi}}{\partial v}(u, 0)=S_{1}(u) \tilde{\varphi}(u, 0)=S_{1}(u) \varphi(u, 0) .
$$

Finally, introducing

$$
\begin{aligned}
\tilde{\varphi}(u, v, w) & :=\mathbb{E}\left(e^{i u X_{\Phi}(0)+i v \partial_{1} X_{\Phi}(0)+i w\left[\partial_{2}^{2} X_{\Phi}(0)-\partial_{1}^{2} X_{\Phi}(0)\right]}\right) \\
& =\exp \left(\lambda \int_{\mathbb{R}^{d} \times \mathbb{R}^{2}}\left(e^{i\left[u g_{m}(x)+v \partial_{1} g_{m}(x)+w\left[\partial_{2}^{2} g_{m}(x)-\partial_{1}^{2} g_{m}(x)\right]\right]}-1\right) F(d m) d x\right),
\end{aligned}
$$

since $\tilde{\tilde{\varphi}}(u, v, w)=\tilde{\tilde{\varphi}}(u,-v, w)$ we also have

$$
\tilde{\tilde{\varphi}}(u, v, w)=\mathbb{E}\left(e^{i u X_{\Phi}(0)+i w\left[\partial_{2}^{2} X_{\Phi}(0)-\partial_{1}^{2} X_{\Phi}(0)\right]} \cos \left(\partial_{1} X_{\Phi}(0) v\right)\right) .
$$

Hence,

$$
\mathbb{E}\left(h_{u}\left(X_{\Phi}(0)\right)\left[\partial_{2}^{2} X_{\Phi}(0)-\partial_{1}^{2} X_{\Phi}(0)\right] \cos \left(\partial_{1} X_{\Phi}(0) v\right)\right)=-i \frac{\partial \tilde{\tilde{\varphi}}}{\partial w}(u, v, 0)=S_{2}(u, v) \varphi(u, v) .
$$

Example 1: We will consider here the example of a smooth isotropic shot noise random field given by

$$
\forall x \in \mathbb{R}^{2}, \quad X_{\Phi}(x)=\sum_{i \in I} \beta_{i} g\left(x-x_{i}\right),
$$


where $\left\{x_{i}\right\}_{i \in I}$ is a Poisson point process of intensity $\lambda$ on $\mathbb{R}^{2}$, the $\beta_{i}$ are independent weights following an exponential distribution of parameter $\mu$ on $\mathbb{R}_{+}$and $g$ is the function given by $g(x)=\exp \left(-a\|x\|^{2} / 2\right)$, with $a>0$ a fixed number. As previously, for $u \in \mathbb{R}$, let $h_{u}$ be the function defined on $\mathbb{R}$ by $t \mapsto e^{i u t}$. When $\beta$ follows an exponential distribution of parameter $\mu$, we can compute its characteristic function

$$
\widehat{F_{\beta}}(u)=\mathbb{E}\left(e^{i u \beta}\right)=\int_{0}^{+\infty} \mu e^{i u s} e^{-\mu s} d s=\frac{\mu}{\mu-i u} .
$$

And we also have that

$$
\mathbb{E}\left(\beta e^{i u \beta}\right)=-i \frac{\partial}{\partial u} \mathbb{E}\left(e^{i u \beta}\right)=\frac{\mu}{(\mu-i u)^{2}} .
$$

Then, denoting by $\varphi(u, v)$ the joint characteristic function of $X_{\Phi}(0)$ and $\partial_{1} X_{\Phi}(0)$, we have

$$
\begin{aligned}
\varphi(u, v) & =\exp \left(\lambda \iint\left[e^{i u \beta g(x)+i v \beta \partial_{1} g(x)}-1\right] F(d \beta) d x\right) \\
& =\exp \left(\lambda \int_{0}^{2 \pi} \int_{0}^{+\infty} \frac{i(u-a v r \cos \theta) e^{-a r^{2} / 2}}{\mu-i(u-a v r \cos \theta) e^{-a r^{2} / 2}} r d r d \theta\right) .
\end{aligned}
$$

This allows us to compute explicitly the law of $X_{\Phi}(0)$, since

$$
\varphi(u, 0)=\mathbb{E}\left(e^{i u X_{\Phi}(0)}\right)=\exp \left(\lambda \int_{0}^{2 \pi} \int_{0}^{+\infty} \frac{i u e^{-a r^{2} / 2}}{\mu-i u e^{-a r^{2} / 2}} r d r d \theta\right)=\left(\frac{\mu}{\mu-i u}\right)^{2 \pi \lambda / a} .
$$

This shows that $X_{\Phi}(0)$ follows a Gamma distribution of parameters $\mu$ and $2 \pi \lambda / a$. We can also compute the level perimeter integral, and get, according to Theorem 3,

$$
\overline{\mathrm{LP}_{X_{\Phi}}}\left(h_{u}\right)=\int_{0}^{+\infty} \frac{1}{v} \varphi(u, v) S_{0}(u, v) d v,
$$

with

$$
S_{0}(u, v)=-i \lambda \int_{0}^{+\infty} \int_{0}^{2 \pi} \frac{\mu a r^{2} \cos (\theta) e^{-a r^{2} / 2}}{\left(\mu-i(u-a v r \cos \theta) e^{-a r^{2} / 2}\right)^{2}} d \theta d r .
$$

For the level total curvature integral, the first term can be made explicit, and it is given by (see again Theorem 3) $\overline{\operatorname{LTC}_{X_{\Phi}}^{(1)}}\left(h_{u}\right)=S_{1}(u) \varphi(u, 0)$, where

$$
S_{1}(u)=-\lambda \iint \beta \partial_{1}^{2} g(x) e^{i u \beta g(x)} F(d \beta) d x=:-\lambda I_{1} .
$$

The integral $I_{1}$ can be computed as follows:

$$
\begin{aligned}
I_{1} & :=\iint \beta \partial_{1}^{2} g(x) e^{i u \beta g(x)} F(d \beta) d x=\int_{\mathbb{R}^{2}} a\left(a x_{1}^{2}-1\right) g\left(x_{1}, x_{2}\right) \frac{\mu}{\left(\mu-i u g\left(x_{1}, x_{2}\right)\right)^{2}} d x_{1} d x_{2} \\
& =\int_{0}^{2 \pi} \int_{0}^{+\infty} a\left(a r^{2} \cos ^{2} \theta-1\right) e^{-a r^{2} / 2} \frac{\mu}{\left(\mu-i u e^{-a r^{2} / 2}\right)^{2}} r d r d \theta=-2 \pi\left(\frac{1}{i u} \log \frac{\mu-i u}{\mu}+\frac{1}{\mu-i u}\right) .
\end{aligned}
$$

This finally leads to

$$
\overline{\operatorname{LTC}_{X_{\Phi}}^{(1)}}\left(h_{u}\right)=2 \pi \lambda\left(\frac{\mu}{\mu-i u}\right)^{2 \pi \lambda / a}\left(\frac{1}{i u} \log \frac{\mu-i u}{\mu}+\frac{1}{\mu-i u}\right) .
$$


Let us note that, writing $\nu=2 \pi \lambda / a>0$, this term corresponds to the Fourier transform of the function $2 \pi \lambda f_{\nu}$, where

$$
f_{\nu}(t)=\left(\frac{(\mu t)^{\nu}}{\Gamma(\nu+1)} e^{-\mu t}-\int_{0}^{\mu t}(\psi(\nu)-\log (s)) \frac{1}{\Gamma(\nu)} s^{\nu-1} e^{-s} d s\right) \mathbf{I}_{t>0},
$$

using the fact that the inverse Laplace transform of $p \mapsto p^{-\nu} \log (p)$ is given by $s \mapsto \frac{s^{\nu-1}}{\Gamma(\nu)}(\psi(\nu)-\log (s))$ (see [18] p.251), where $\psi$ is the logarithmic derivative of the $\Gamma$ function.

For the second term, $\overline{\operatorname{LTC}_{X_{\Phi}}^{(2)}}\left(h_{u}\right)$, we use the formula given by

$$
\overline{\operatorname{LTC}_{X_{\Phi}}^{(2)}}\left(h_{u}\right)=\int_{0}^{+\infty} \frac{1}{v} \varphi(u, v) S_{2}(u, v) d v,
$$

where $\varphi(u, v)$ was computed above, and $S_{2}$ is given here by

$$
S_{2}(u, v)=-\lambda \int_{0}^{+\infty} \int_{0}^{2 \pi} \frac{\mu a^{2} r^{3} \cos (2 \theta) e^{-a r^{2} / 2}}{\left(\mu-i(u-a v r \cos \theta) e^{-a r^{2} / 2}\right)^{2}} d \theta d r .
$$

All these integrals can be efficiently numerically computed (using Matlab for instance). Some results, comparing empirical and theoretical curves, are shown on Figure 5.

Example 2: This example is the same as the previous one, except that the $\beta_{i}$ follow now a Laplace distribution of parameter $\mu$, and the shot noise random field has therefore the additional property of being symmetric. Here,

$$
\widehat{F_{\beta}}(u)=\mathbb{E}\left(e^{i u \beta}\right)=\int_{-\infty}^{+\infty} \frac{1}{2} \mu e^{i u s} e^{-\mu|s|} d s=\frac{\mu^{2}}{\mu^{2}+u^{2}} .
$$

And we also have that

$$
\mathbb{E}\left(\beta e^{i u \beta}\right)=-i \frac{\partial}{\partial u} \mathbb{E}\left(e^{i u \beta}\right)=\frac{2 i u \mu^{2}}{\left(\mu^{2}+u^{2}\right)^{2}} .
$$

Then, denoting again by $\varphi(u, v)$ the joint characteristic function of $X_{\Phi}(0)$ and $\partial_{1} X_{\Phi}(0)$, we have

$$
\begin{aligned}
\varphi(u, v) & =\exp \left(\lambda \iint\left[e^{i u \beta g(x)+i v \beta \partial_{1} g(x)}-1\right] F(d \beta) d x\right) \\
& =\exp \left(-\lambda \int_{0}^{2 \pi} \int_{0}^{+\infty} \frac{(u-a v r \cos \theta)^{2} e^{-a r^{2}}}{\mu^{2}+(u-a v r \cos \theta)^{2} e^{-a r^{2}}} r d r d \theta\right) .
\end{aligned}
$$

This allows us to compute explicitly the law of $X_{\Phi}(0)$, since

$$
\varphi(u, 0)=\mathbb{E}\left(e^{i u X_{\Phi}(0)}\right)=\exp \left(-\lambda \int_{0}^{2 \pi} \int_{0}^{+\infty} \frac{u^{2} e^{-a r^{2}}}{\mu^{2}+u^{2} e^{-a r^{2}}} r d r d \theta\right)=\left(\frac{\mu^{2}}{\mu^{2}+u^{2}}\right)^{\pi \lambda / a} .
$$

This shows that $X_{\Phi}(0)$ follows a symmetric generalized Laplace distribution of parameters $\mu$ and $\pi \lambda / a$. We can also compute the level perimeter integral, and get, according to Theorem 3 ,

$$
\overline{\mathrm{LP}_{X}}\left(h_{u}\right)=\int_{0}^{+\infty} \frac{1}{v} \varphi(u, v) S_{0}(u, v) d v,
$$


with now

$$
S_{0}(u, v)=\lambda \int_{0}^{+\infty} \int_{0}^{2 \pi} \frac{2 \mu^{2} a r^{2} \cos (\theta)(u-a v r \cos \theta) e^{-a r^{2}}}{\left(\mu^{2}+(u-a v r \cos \theta)^{2} e^{-a r^{2}}\right)^{2}} d \theta d r
$$

For the level total curvature integral, the first term can also be made explicit, and it is given by (see again Theorem 3) $\overline{\operatorname{LTC}_{X_{\Phi}}^{(1)}}\left(h_{u}\right)=S_{1}(u) \varphi(u, 0)$ with

$$
S_{1}(u)=-\lambda \iint \beta \partial_{1}^{2} g(x) e^{i u \beta g(x)} F(d \beta) d x=:-\lambda I_{1} .
$$

Here the integral $I_{1}$ can be computed as follows:

$$
\begin{aligned}
I_{1} & :=\iint \beta \partial_{1}^{2} g(x) e^{i u \beta g(x)} F(d \beta) d x=\int_{\mathbb{R}^{2}} a\left(a x_{1}^{2}-1\right) g\left(x_{1}, x_{2}\right) \frac{2 i u g\left(x_{1}, x_{2}\right) \mu^{2}}{\left(\mu^{2}+u^{2} g\left(x_{1}, x_{2}\right)^{2}\right)^{2}} d x_{1} d x_{2} \\
& =\int_{0}^{2 \pi} \int_{0}^{+\infty} 2 i u a \mu^{2} \frac{\left(a r^{2} \cos ^{2} \theta-1\right) e^{-a r^{2}}}{\left(\mu^{2}+u^{2} e^{\left.-a r^{2}\right)^{2}}\right.} r d r d \theta=\frac{i \pi}{u}\left(\log \frac{\mu^{2}+u^{2}}{\mu^{2}}-\frac{2 u^{2}}{\mu^{2}+u^{2}}\right) .
\end{aligned}
$$

This finally leads to

$$
\overline{\operatorname{LTC}_{X_{\Phi}}^{(1)}}\left(h_{u}\right)=\frac{i \lambda \pi}{u}\left(\frac{\mu^{2}}{\mu^{2}+u^{2}}\right)^{\pi \lambda / a}\left(\log \frac{\mu^{2}}{\mu^{2}+u^{2}}+\frac{2 u^{2}}{\mu^{2}+u^{2}}\right) .
$$

For the second term, $\overline{\operatorname{LTC}_{X_{\Phi}}^{(2)}}\left(h_{u}\right)$, we use the formula given by

$$
\overline{\operatorname{LTC}_{X_{\Phi}}^{(2)}}\left(h_{u}\right)=\int_{0}^{+\infty} \frac{1}{v} \varphi(u, v) S_{2}(u, v) d v,
$$

where $\varphi(u, v)$ was computed above and $S_{2}$ is given here by

$$
S_{2}(u, v)=i \lambda \int_{0}^{+\infty} \int_{0}^{2 \pi} \frac{-2 \mu^{2} a^{2} r^{3} \cos (2 \theta)(u-a v r \cos \theta) e^{-a r^{2}}}{\left(\mu^{2}+(u-a v r \cos \theta)^{2} e^{-a r^{2}}\right)^{2}} d \theta d r .
$$

Again, all these integrals can be efficiently numerically computed (using Matlab for instance). Some results, comparing empirical and theoretical curves, are shown on Figure 5. Notice that the theoretical curves (red curves) present some oscillations that are due to the numerical approximations we have made (indeed to numerically compute the integrals, we had to truncate them, and since the Fourier transform is involved, this results in some oscillations). 

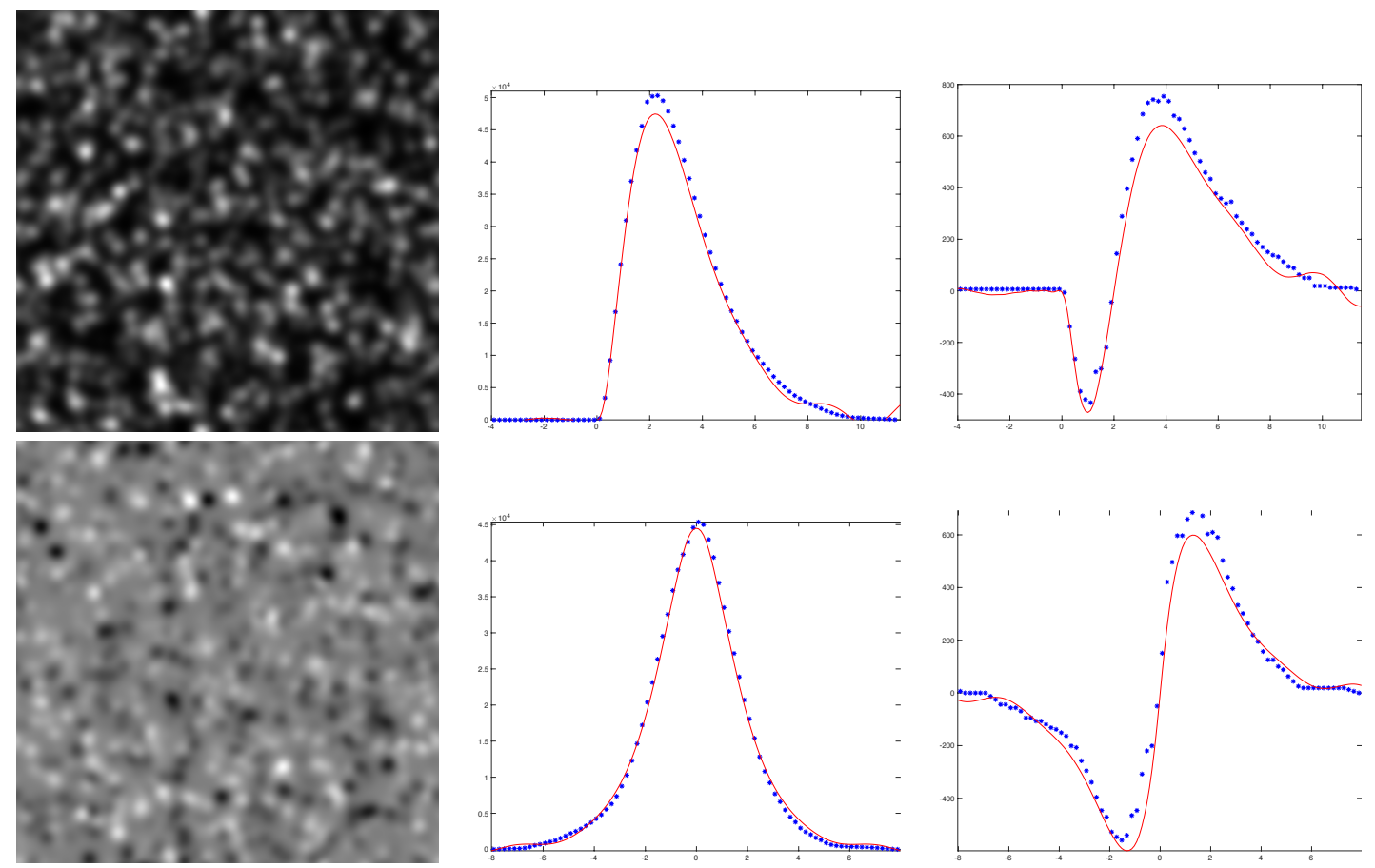

FIG 5. First line: on the left, a sample of the shot noise of Example 1; in the middle: its empirical perimeter $t \mapsto \operatorname{Per}\left(E_{X}(t)\right)$ (blue stars), and the theoretical curve $t \mapsto \overline{\operatorname{Per}\left(E_{X}(t)\right)}$ (red curve) obtained numerically by inverting the Fourier transform of $u \mapsto \overline{L P_{X_{\Phi}}}\left(h_{u}\right)$; on the right: the empirical total curvature $t \mapsto \operatorname{TC}\left(E_{X}(t)\right)$ (blue stars), and the theoretical curve $t \mapsto \overline{\mathrm{TC}\left(E_{X}(t)\right)}$ (red curve) obtained numerically by inverting the Fourier transform of $u \mapsto \overline{L T C_{X_{\Phi}}}\left(h_{u}\right)$. Second line: same experiments but with the shot noise of Example 2.

\section{Elementary functions and elementary shot noise random fields}

\subsection{Elementary functions}

We introduce now the class of elementary functions that are piecewise constant functions defined more precisely in the following way.

Definition 5 (Elementary function). We say that a function $f$ defined on $\mathbb{R}^{2}$ is an elementary function if there exist an integer $n$, non-zero real numbers $a_{1}, \ldots, a_{n}$ and $n$ elementary sets (Definition 2) denoted $A_{1}, \ldots, A_{n}$ that are in generic position (Definition 3), such that

$$
f=\sum_{k=1}^{n} a_{k} \mathbf{I}_{A_{k}}
$$

Let us remark that since $\mathbb{R}^{2}$ itself is an elementary set, constant functions on $\mathbb{R}^{2}$ are elementary functions.

In Section 2 we have seen the definitions of regular points and corner points of a curve. We here extend these definitions to elementary functions.

We first introduce some notations. For a point $x \in U$ and a real positive number $\rho$, we recall that $B_{\rho}(x)$ denotes the open ball of radius $\rho$ and center $x$. For an oriented simple piecewise 
$C^{2}$ curve $\gamma$ and a point $x$ on $\gamma$, then for $\rho$ small enough, $B_{\rho}(x) \backslash \gamma$ is made of two connected components. These two "half-balls" are respectively denoted by $B_{\rho}^{+}(x, \gamma)$ and $B_{\rho}^{-}(x, \gamma)$. The half-ball $B_{\rho}^{+}(x, \gamma)$ is the component that is on the side of the normal $\nu_{\gamma}$ to $\gamma$.

Let us make the important and useful remark that the discontinuity set $\mathcal{S}_{f}$ of an elementary function $f=\sum_{i} a_{i} \mathbf{I}_{A_{i}}$ is equal to the union of the $\partial A_{i}$ :

$$
\mathcal{S}_{f}=\cup_{i} \partial A_{i}
$$

Indeed the inclusion $\mathcal{S}_{f} \subset \cup_{i} \partial A_{i}$ is obvious, and the reverse inclusion holds since all the $a_{i}$ are non-zero. Therefore a point $x$ on the discontinuity set belongs else to a single $\partial A_{i}$ and it is then a regular discontinuity point or a corner discontinuity point, or it belongs to two boundaries $\partial A_{i}$ and $\partial A_{j}$ and it is then called an intersection discontinuity point. See Figure 6 for a schematic representation of the three types of points.
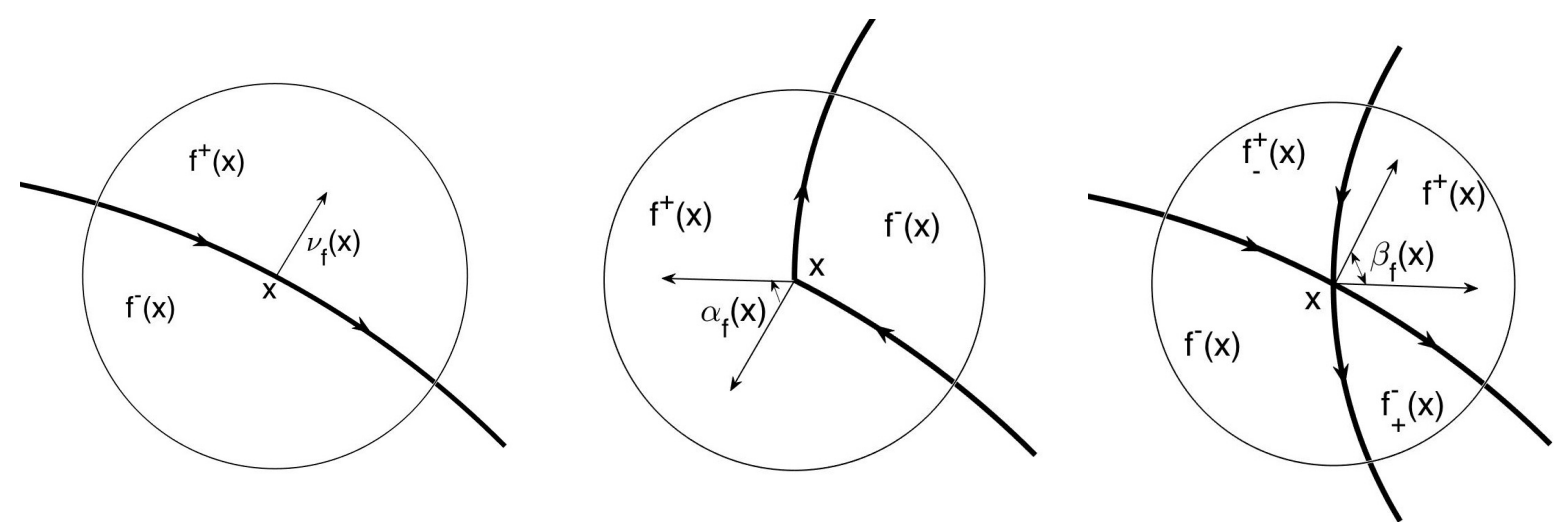

FIG 6. The three types of points of the discontinuity set of an elementary function. From left to right: a regular discontinuity point, a corner discontinuity point and an intersection discontinuity point.

To be more rigorous, here are the definitions and some notations.

- A point $x$ is a regular discontinuity point for $f$ if there exists $\rho>0$ such that $\mathcal{S}_{f} \cap B_{\rho}(x)$ is a simple $C^{2}$ oriented curve $\gamma$ separating the ball $B_{\rho}(x)$ in two half-balls $B_{\rho}^{+}(x, \gamma)$ and $B_{\rho}^{-}(x, \gamma)$, and there are two real numbers $f^{+}(x)>f^{-}(x)$ such that $f(y)=f^{+}(x)$ for all $y \in B_{\rho}^{+}(x, \gamma)$ and $f(y)=f^{-}(x)$ for all $y \in B_{\rho}^{-}(x, \gamma)$. We will denote

$$
\nu_{f}(x)=\nu_{\gamma}(x) \quad \text { and } \quad \kappa_{f}(x)=\kappa_{\gamma}(x) .
$$

- A point $x$ is a corner discontinuity point for $f$ if there exists $\rho>0$ such that $\mathcal{S}_{f} \cap B_{\rho}(x)$ is a simple piecewise $C^{2}$ oriented curve $\gamma$ having only one corner at $x$. We write $\alpha_{f}(x) \in$ $(-\pi, \pi)$ the turning angle of $\gamma$ at $x$. As for regular discontinuity points, $\gamma$ separates the ball $B_{\rho}(x)$ in two half-balls $B_{\rho}^{+}(x, \gamma)$ and $B_{\rho}^{-}(x, \gamma)$, and moreover there exist two real numbers $f^{+}(x)>f^{-}(x)$ such that $f(y)=f^{+}(x)$ for all $y \in B_{\rho}^{+}(x, \gamma)$ and $f(y)=f^{-}(x)$ for all $y \in B_{\rho}^{-}(x, \gamma)$. The turning angle at such a corner point is denoted

$$
\alpha_{f}(x)=\alpha_{\gamma}(x)
$$

- A point $x$ is an intersection discontinuity point for $f$ if there exists $\rho>0$ such that $\mathcal{S}_{f} \cap B_{\rho}(x)$ is the union of two different simple and oriented $C^{2}$ curves $\gamma_{1}$ and $\gamma_{2}$ in generic position and such that $\{x\}=\gamma_{1} \cap \gamma_{2}$. Each curve separates the ball in two half-balls, and there exist 
4 real numbers $f^{-}(x) \leq f_{-}^{+}(x), f_{+}^{-}(x) \leq f^{+}(x)$ with at least 3 different values, such that $f=f^{-}(x)$ on $B_{\rho}^{-}\left(x, \gamma_{1}\right) \cap B_{\rho}^{-}\left(x, \gamma_{2}\right) ; f=f_{+}^{-}(x)$ on $B_{\rho}^{-}\left(x, \gamma_{1}\right) \cap B_{\rho}^{+}\left(x, \gamma_{2}\right) ; f=f_{-}^{+}(x)$ on $B_{\rho}^{+}\left(x, \gamma_{1}\right) \cap B_{\rho}^{-}\left(x, \gamma_{2}\right)$ and $f=f^{+}(x)$ on $B_{\rho}^{+}\left(x, \gamma_{1}\right) \cap B_{\rho}^{+}\left(x, \gamma_{2}\right)$. And we define

$$
\begin{aligned}
\beta_{f}(x) & =d_{S^{1}}\left(\nu_{\gamma_{1}}(x), \nu_{\gamma_{2}}(x)\right) \\
& =\min \left(\left|\operatorname{Arg} \nu_{\gamma_{1}}(x)-\operatorname{Arg} \nu_{\gamma_{2}}(x)\right|, 2 \pi-\left|\operatorname{Arg} \nu_{\gamma_{1}}(x)-\operatorname{Arg} \nu_{\gamma_{2}}(x)\right|\right) \in(0, \pi),
\end{aligned}
$$

the geodesic distance between $\nu_{\gamma_{1}}(x)$ and $\nu_{\gamma_{2}}(x)$ on $S^{1}$.

Let us remark that for an elementary function, at an intersection discontinuity point $x$, we must also have $f_{-}^{+}(x)+f_{+}^{-}(x)=f^{-}(x)+f^{+}(x)$. Indeed one can write locally $f(x)=f_{1}(x)+f_{2}(x)$ with $x$ a regular discontinuity point for both $f_{1}$ and $f_{2}$. Hence $f^{+}(x)=f_{1}^{+}(x)+f_{2}^{+}(x)$ and $f^{-}(x)=f_{1}^{-}(x)+f_{2}^{-}(x)$ so that $f^{-}(x)+f^{+}(x)=f_{-}^{+}(x)+f_{+}^{-}(x)$.

Definition 6 (Elementary function on $U$ ). Let $U$ be an open set of $\mathbb{R}^{2}$, we say that a function $f$ defined on $U$ is an elementary function on $U$ if there exists $\tilde{f}$ an elementary function such that for all $x \in U$ we have $f(x)=\tilde{f}(x)$.

Proposition 4. Let $f$ be an elementary function, and let $\mathcal{R}_{f}, \mathcal{C}_{f}$ and $\mathcal{I}_{f}$ denote respectively the set of regular, corner and intersection discontinuity points of $f$. Then for all $t \in \mathbb{R}, E_{f}(t)$ is an elementary set (in the sense of Definition 2). Moreover, if $U$ is an open subset of $\mathbb{R}^{2}$ and $f$ is an elementary function on $U$, then $f$ is of finite total variation and finite level total curvature integral on $U$ with

$$
V_{f}(U)=\|D f(U)\|=\int_{\mathcal{R}_{f} \cap U}\left[f^{+}(x)-f^{-}(x)\right] \mathcal{H}^{1}(d x) \text { and }
$$

$$
\begin{aligned}
& \operatorname{LTaC}_{f}(U)=\int_{\mathcal{R}_{f} \cap U}\left[f^{+}(x)-f^{-}(x)\right]\left|\kappa_{f}(x)\right| \mathcal{H}^{1}(d x)+\sum_{x \in \mathcal{C}_{f} \cap U}\left[f^{+}(x)-f^{-}(x)\right]\left|\alpha_{f}(x)\right| \\
& \quad+\sum_{x \in \mathcal{I}_{f} \cap U}\left[f^{+}(x)-\max \left(f_{-}^{+}(x), f_{-}^{-}(x)\right)+\min \left(f_{-}^{+}(x), f_{-}^{-}(x)\right)-f^{-}(x)\right] \beta_{f}(x)<+\infty .
\end{aligned}
$$

If $h$ is a bounded continuous function on $\mathbb{R}$, and $H$ is a primitive of $h$ (for instance $H(t)=$ $\left.\int_{0}^{t} h(u) d u\right)$, then the level perimeter integral and the total curvature integral of $f$ are given by

$$
\begin{gathered}
\operatorname{LP}_{f}(h, U)=\int_{\mathcal{R}_{f} \cap U}\left[H\left(f^{+}(x)\right)-H\left(f^{-}(x)\right)\right] \mathcal{H}^{1}(d x) \\
\operatorname{LTC}_{f}(h, U)=\int_{\mathcal{R}_{f} \cap U}\left[H\left(f^{+}(x)\right)-H\left(f^{-}(x)\right)\right] \kappa_{f}(x) \mathcal{H}^{1}(d x)+\sum_{x \in \mathcal{C}_{f} \cap U}\left[H\left(f^{+}(x)\right)-H\left(f^{-}(x)\right)\right] \alpha_{f}(x) \\
+\sum_{x \in \mathcal{I}_{f} \cap U}\left[H\left(f^{+}(x)\right)+H\left(f^{-}(x)\right)-H\left(f_{-}^{+}(x)\right)-H\left(f_{+}^{-}(x)\right)\right] \beta_{f}(x) .
\end{gathered}
$$

In particular, when $h=1$, we get $\operatorname{LP}_{f}(1, U)=V_{f}(U)$ and

$$
\operatorname{LTC}_{f}(U)=\int_{\mathcal{R}_{f} \cap U}\left[f^{+}(x)-f^{-}(x)\right] \kappa_{f}(x) \mathcal{H}^{1}(d x)+\sum_{x \in \mathcal{C}_{f} \cap U}\left[f^{+}(x)-f^{-}(x)\right] \alpha_{f}(x) .
$$


Remark: Notice that Equation (19) doesn't involve the intersection discontinuity points of the function $f$. In particular, this implies that if $f$ and $g$ are two elementary functions such that their elementary sets are all in generic position (which is equivalent to say that their discontinuity sets $\mathcal{S}_{f}$ and $\mathcal{S}_{g}$ are in generic position), then $f+g$ is an elementary function and we have

$$
V_{f+g}(U)=V_{f}(U)+V_{g}(U) \quad \text { and } \quad \operatorname{LTC}_{f+g}(U)=\operatorname{LTC}_{f}(U)+\operatorname{LTC}_{g}(U),
$$

for any bounded open set $U$. This result is quite striking, but we have to underline that it doesn't hold in general for other classes of functions. Indeed, in the previous section we have considered smooth functions $f$ and the formula for the level perimeter and the total curvature integral were obviously not linear in $f$. Here the main point is that when $f$ and $g$ are elementary functions in a generic position, then the two measures $D f$ and $D g$ are mutually singular, which is not the case in general for any two functions $f$ and $g$.

Proof. Since $f$ is an elementary function, we can write $f=\sum_{j=1}^{n} a_{j} \mathbf{I}_{A_{j}}$ with $a_{1}, \ldots, a_{n}$ real numbers and $A_{1}, \ldots, A_{n}$ elementary sets in generic position. Let us assume that $m=\operatorname{Card}\left(f\left(\mathbb{R}^{2}\right)\right) \geq$ 2. Otherwise, if $m=1$, then for all $t \in \mathbb{R}, E_{f}(t)=\emptyset$ or $\mathbb{R}^{2}$ and therefore $\operatorname{Per}\left(E_{f}(t), U\right)=$ $\mathrm{TaC}\left(\partial E_{f}(t), U\right)=\mathrm{TC}\left(\partial E_{f}(t), U\right)=0$. In the following we denote the values of $f$ by $v_{1}<$ $\ldots<v_{m}$ and set $v_{0}=-\infty$. We first remark that $E_{f}(t)=\emptyset$ for $t>v_{m}, E_{f}(t)=\mathbb{R}^{2}$ for $t \leq v_{1}$ and $E_{f}(t)=E_{f}\left(v_{i}\right)$ for $v_{i-1}<t \leq v_{i}$ and $2 \leq i \leq m$. The set of discontinuity points is given by $\mathcal{S}_{f}=\cup_{i=2}^{m} \partial E_{f}\left(v_{i}\right)=\cup_{j=1}^{n} \partial A_{j}$. Since the sets $\left(A_{j}\right)_{1 \leq j \leq n}$ are in generic position, each $\partial E_{f}\left(v_{i}\right)$ is a piecewise regular curve and therefore $E_{f}\left(v_{i}\right)$ is an elementary set with $\operatorname{Per}\left(E_{f}\left(v_{i}\right), U\right)=\mathcal{H}^{1}\left(\mathcal{R}_{f} \cap \Gamma_{i}\right)$ for $\Gamma_{i}:=\partial E_{f}\left(v_{i}\right) \cap U$. Now, for $x \in \Gamma_{i}$ we must have $f^{+}(x) \geq v_{i}$ and $f^{-}(x)<v_{i}$ and since $\Gamma_{i} \subset \mathcal{S}_{f}$, we can write

$$
\Gamma_{i}=\left(\Gamma_{i} \cap \mathcal{R}_{f}\right) \cup\left(\Gamma_{i} \cap \mathcal{C}_{f}\right) \cup\left(\Gamma_{i} \cap \mathcal{I}_{f}\right) .
$$

If $x \in \mathcal{R}_{f}$ or $x \in \mathcal{C}_{f}$ then there exists a unique $j$ such that $x \in \partial A_{j}$ is a regular or corner point of $\partial A_{j}$ with $\kappa_{\Gamma_{i}}(x)=\kappa_{f}(x)$ or $\alpha_{\Gamma_{i}}(x)=\alpha_{f}(x)$. Otherwise, if $x \in \mathcal{I}_{f}$ there exit $j \neq j^{\prime}$ such that $x \in \partial A_{j} \cap \partial A_{j^{\prime}}$ and $x$ may become a corner point or a regular point for $\Gamma_{i}$. Indeed, if $v_{i} \leq \min \left(f_{-}^{+}(x), f_{+}^{-}(x)\right)$, then $x \in \mathcal{C}_{\Gamma_{i}}$ with $\alpha_{\Gamma_{i}}(x)=-\beta_{f}(x)$. Without loss of generality we may assume that $f_{-}^{+}(x) \leq f_{+}^{-}(x)$. If $f_{-}^{+}(x)<v_{i} \leq f_{+}^{-}(x)$ then $x$ is not a corner point of $\Gamma_{i}$ but a regular point. If $v_{i}>\max \left(f_{-}^{+}(x), f_{-}^{-}(x)\right)$, then $x$ is again a corner point of $\Gamma_{i}$ with $\alpha_{\Gamma_{i}}(x)=\beta_{f}(x)$. Therefore,

$$
\begin{aligned}
\operatorname{TaC}\left(\Gamma_{i}, U\right)= & \int_{\Gamma_{i} \cap \mathcal{R}_{f}}\left|\kappa_{f}(x)\right| \mathcal{H}^{1}(d x)+\sum_{x \in \Gamma_{i} \cap \mathcal{C}_{f}}\left|\alpha_{f}(x)\right| \\
& +\sum_{x \in \Gamma_{i} \cap \mathcal{I}_{f}} \beta_{f}(x)\left(\mathbb{I}_{v_{i}>\max \left(f_{-}^{+}(x), f_{-}^{-}(x)\right)}+\mathbb{I}_{v_{i} \leq \min \left(f_{-}^{+}(x), f_{-}^{-}(x)\right)}\right),
\end{aligned}
$$

while

$$
\begin{aligned}
\operatorname{TC}\left(\Gamma_{i}, U\right)= & \int_{\Gamma_{i} \cap \mathcal{R}_{f}} \kappa_{f}(x) \mathcal{H}^{1}(d x)+\sum_{x \in \Gamma_{i} \cap \mathcal{C}_{f}} \alpha_{f}(x) \\
& +\sum_{x \in \Gamma_{i} \cap \mathcal{I}_{f}} \beta_{f}(x)\left(\mathbb{I}_{v_{i}>\max \left(f_{-}^{+}(x), f_{-}^{-}(x)\right)}-\mathbb{I}_{v_{i} \leq \min \left(f_{-}^{+}(x), f_{-}^{-}(x)\right)}\right),
\end{aligned}
$$

and

$$
\operatorname{Per}\left(\Gamma_{i}, U\right)=\int_{\Gamma_{i} \cap \mathcal{R}_{f}} \mathcal{H}^{1}(d x) .
$$


Now, let $h$ be a continuous bounded function on $\mathbb{R}$ and $H$ a primitive of $h$. For the level perimeter integral we have

$$
\begin{aligned}
\operatorname{LP}_{f}(h, U) & =\int_{\mathbb{R}} h(t) \operatorname{Per}\left(\partial E_{f}(t), U\right) d t=\sum_{i=2}^{m} \int_{v_{i-1}}^{v_{i}} h(t) \operatorname{Per}\left(\Gamma_{i}, U\right) d t \\
& =\sum_{i=2}^{m}\left[H\left(v_{i}\right)-H\left(v_{i-1}\right)\right] \operatorname{Per}\left(\Gamma_{i}, U\right)=\int_{\mathcal{R}_{f}} \sum_{i=2}^{m}\left[H\left(v_{i}\right)-H\left(v_{i-1}\right)\right] \mathbb{I}_{\Gamma_{i}}(x) \mathcal{H}^{1}(d x) .
\end{aligned}
$$

When $x \in \mathcal{R}_{f} \cap U=\cup_{i=2}^{m} \Gamma_{i}$, we denote by $i(x)$, (resp. $j(x) \geq i(x)$ ), the minimal (resp. maximal) index $i=2, \ldots, m$ such that $x \in \Gamma_{i}$ and then $f^{-}(x)=v_{i(x)-1}$, (resp. $\left.f^{+}(x)=v_{j(x)}\right)$. It follows that

$$
\begin{aligned}
\sum_{i=2}^{m}\left[H\left(v_{i}\right)-H\left(v_{i-1}\right)\right] \mathbb{I}_{\Gamma_{i}}(x) & =\sum_{i=i(x)}^{j(x)}\left[H\left(v_{i}\right)-H\left(v_{i-1}\right)\right] \\
& =H\left(v_{j(x)}\right)-H\left(v_{i(x)-1}\right) \\
& =H\left(f^{+}(x)\right)-H\left(f^{-}(x)\right) .
\end{aligned}
$$

Therefore we get

$$
\operatorname{LP}_{f}(h, U)=\int_{\mathcal{R}_{f}}\left(H\left(f^{+}(x)\right)-H\left(f^{-}(x)\right)\right) \mathcal{H}^{1}(d x) .
$$

Now, for the total absolute curvature, we have

$$
\int_{\mathbb{R}} h(t) \operatorname{TaC}\left(\partial E_{f}(t), U\right) d t=\sum_{i=2}^{m} \int_{v_{i-1}}^{v_{i}} h(t) \operatorname{TaC}\left(\Gamma_{i}, U\right) d t=\sum_{i=2}^{m}\left[H\left(v_{i}\right)-H\left(v_{i-1}\right)\right] \operatorname{TaC}\left(\Gamma_{i}, U\right) .
$$

Then, using the above formula for $\operatorname{TaC}\left(\Gamma_{i}, U\right)$, we get the sum of three terms. The first one is given by

$$
\sum_{i=2}^{m}\left[H\left(v_{i}\right)-H\left(v_{i-1}\right)\right] \int_{\mathcal{R}_{f} \cap \Gamma_{i}}\left|\kappa_{f}(x)\right| \mathcal{H}^{1}(d x)=\int_{\mathcal{R}_{f}}\left|\kappa_{f}(x)\right| \sum_{i=2}^{m}\left[H\left(v_{i}\right)-H\left(v_{i-1}\right)\right] \mathbf{I}_{\Gamma_{i}}(x) \mathcal{H}^{1}(d x) .
$$

Using Equation (20), this first term is equal to

$$
\int_{\mathcal{R}_{f} \cap U}\left[H\left(f^{+}(x)\right)-H\left(f^{-}(x)\right)\right]\left|\kappa_{f}(x)\right| \mathcal{H}^{1}(d x) .
$$

Similarly, the second term is equal to

$$
\sum_{i=2}^{m}\left[H\left(v_{i}\right)-H\left(v_{i-1}\right)\right] \sum_{x \in \Gamma_{i} \cap \mathcal{C}_{f}}\left|\alpha_{f}(x)\right|=\sum_{x \in \mathcal{C}_{f} \cap U}\left[H\left(f^{+}(x)\right)-H\left(f^{-}(x)\right)\right]\left|\alpha_{f}(x)\right| .
$$

Finally, the third one is given by

$$
\begin{aligned}
\sum_{i=2}^{m}\left[H\left(v_{i}\right)-H\left(v_{i-1}\right)\right] & \sum_{x \in \Gamma_{i} \cap \mathcal{I}_{f}}\left|\beta_{f}(x)\right|\left(\mathbb{I}_{v_{i}>\max \left(f_{-}^{+}(x), f_{-}^{-}(x)\right)}+\mathbf{I}_{v_{i} \leq \min \left(f_{-}^{+}(x), f_{-}^{-}(x)\right)}\right) \\
= & \sum_{x \in \mathcal{I}_{f} \cap U} \beta_{f}(x)\left(\sum_{i=l(x)+1}^{j(x)}\left[H\left(v_{i}\right)-H\left(v_{i-1}\right)\right]+\sum_{i=i(x)}^{k(x)}\left[H\left(v_{i}\right)-H\left(v_{i-1}\right)\right]\right),
\end{aligned}
$$


where we have introduced $k(x)$ and $l(x)$ with $i(x)-1 \leq k(x) \leq l(x) \leq j(x)$ such that $\min \left(f_{-}^{+}(x), f_{-}^{-}(x)\right)=v_{k(x)}$ and $\max \left(f_{-}^{+}(x), f_{-}^{-}(x)\right)=v_{l(x)}$, with the convention that $\sum_{i=l(x)+1}^{j(x)}=$ 0 if $l(x)=j(x)$ and $\sum_{i=i(x)}^{k(x)}=0$ if $k(x)=i(x)-1$. It follows that this third term is equal to

$$
\begin{aligned}
& \sum_{x \in \mathcal{I}_{f} \cap U} \beta_{f}(x)\left(H\left(v_{j(x)}\right)-H\left(v_{l(x)}\right)+\left(H\left(v_{k(x)}\right)-H\left(v_{i(x)-1}\right)\right)\right) \\
= & \left.\sum_{x \in \mathcal{I}_{f}} \beta_{f}(x)\left(H\left(f^{+}(x)\right)-H\left(\max \left(f_{-}^{+}(x), f_{-}^{-}(x)\right)\right)+H\left(\min \left(f_{-}^{+}(x), f_{-}^{-}(x)\right)\right)-H\left(f^{-}(x)\right)\right)\right) .
\end{aligned}
$$

In particular, for $h=1$, we obtain Formula (16). The same computations as above give the result (18) for $\operatorname{LTC}_{f}(h, U)$. Indeed now, the third term is equal to

$$
\begin{aligned}
& \sum_{i=2}^{m}\left[H\left(v_{i}\right)-H\left(v_{i-1}\right)\right] \sum_{x \in \Gamma_{i} \cap \mathcal{I}_{f}} \beta_{f}(x)\left(\mathbb{I}_{v_{i}>\max \left(f_{-}^{+}(x), f_{-}^{-}(x)\right)}-\mathbb{I}_{v_{i} \leq \min \left(f_{-}^{+}(x), f_{-}^{-}(x)\right)}\right) \\
& =\sum_{x \in \mathcal{I}_{f}} \beta_{f}(x)\left(\sum_{i=l(x)+1}^{j(x)}\left[H\left(v_{i}\right)-H\left(v_{i-1}\right)\right]-\sum_{i=i(x)}^{k(x)}\left[H\left(v_{i}\right)-H\left(v_{i-1}\right)\right]\right) \\
& =\sum_{x \in \mathcal{I}_{f}} \beta_{f}(x)\left(H\left(f^{+}(x)\right)+H\left(f^{-}(x)\right)-H\left(f_{-}^{+}(x)\right)-H\left(f_{+}^{-}(x)\right)\right) .
\end{aligned}
$$

Taking again $h=1$ we obtain (19). Finally, let us remark that since the above results depend only on the values of $f$ in $U$, they also hold when $f$ is an elementary function on $U$ in the sense of Definition 6.

\subsection{Level perimeter and level total curvature of an elementary shot noise random field}

As in Section 3.3, we consider here a shot noise random field defined on $\mathbb{R}^{2}$ by

$$
\forall x \in \mathbb{R}^{2}, \quad X_{\Phi}(x)=\sum_{i \in I} g_{m_{i}}\left(x-x_{i}\right),
$$

where $\Phi=\left\{\left(x_{i}, m_{i}\right)\right\}_{i \in I}$ is a Poisson point process on $\mathbb{R}^{2} \times \mathbb{R}^{d}$ of intensity $\lambda \mathcal{L} \times F$, with $\lambda>0$ real, $\mathcal{L}$ the Lebesgue measure on $\mathbb{R}^{2}$ and $F$ a probability measure on $\mathbb{R}^{d}$.

We will first give formulas for the level perimeter and total curvature integrals of $X_{\Phi}$ on an open bounded set $U$ in the case where the $g_{m}$ are elementary functions on $\mathbb{R}^{2}$, then we will compute their expectation. Finally we will give explicit results in the case of weighted indicator functions of random sets, obtained from a deterministic compact elementary set and regular region $D$, by random rotation and dilation. Specific computations for disks with $D=D(0,1)$, and for squares with $D=[0,1]^{2}$ are linked with some recent results on Boolean models.

Throughout the rest of this section we also assume that for $F$-almost every $m \in \mathbb{R}^{d}$, the $g_{m}$ are elementary functions on $\mathbb{R}^{2}$, with compact support, satisfying (11) and such that

$$
\int_{\mathbb{R}^{d}} V_{g_{m}}\left(\mathbb{R}^{2}\right) F(d m)<+\infty \quad \text { and } \quad \int_{\mathbb{R}^{d}} \operatorname{LTaC}_{g_{m}}\left(\mathbb{R}^{2}\right) F(d m)<+\infty,
$$


where $V_{g_{m}}\left(\mathbb{R}^{2}\right)$ and $\mathrm{LTaC}_{g_{m}}\left(\mathbb{R}^{2}\right)$ are defined by (15) and (16) choosing $U=\mathbb{R}^{2}$. For $F$-almost every $m, g_{m}$ is assumed to have a compact support, that can be included in a square $\left[-T_{m}, T_{m}\right]^{2}$ with $T_{m} \in \mathbb{R}_{+}$, and its maximal value $\left\|g_{m}\right\|_{\infty}=\underset{\left[-T_{m}, T_{m}\right]^{2}}{\max }\left|g_{m}\right|$ is finite. We will assume moreover that

$$
\int_{\mathbb{R}^{d}} T_{m}^{2} F(d m)<+\infty \quad \text { and } \quad \int_{\mathbb{R}^{d}}\left\|g_{m}\right\|_{\infty} F(d m)<+\infty
$$

Note that the first assumption of (22) implies that there is a.s. only a finite random number of $g_{m}$, denoted by $N(U)$, contributing to the values of $X_{\Phi}$ on the bounded open set $U \subset(-T, T)^{2}$, for some $T>0$. Indeed, it is clear that

$$
N(U) \leq \#\left\{i ; \bar{U} \cap\left(x_{i}+\left[-T_{m_{i}}, T_{m_{i}}\right]^{2}\right) \neq \emptyset\right\} \leq \#\left\{i ;\left\|x_{i}\right\|_{\infty} \leq\left(T_{m_{i}}+T\right)\right\}
$$

with $\|x\|_{\infty}=\max \left(\left|x_{1}\right|,\left|x_{2}\right|\right)$, for $x \in \mathbb{R}^{2}$. It follows that $\mathbb{E}(N(U)) \leq \lambda \int_{\mathbb{R}^{d}} 4\left(T_{m}+T\right)^{2} F(d m)$. Since $F$ is a finite measure, under $(22)$, we get

$$
\mathbb{E}(N(U))<+\infty .
$$

In the following we will use the notation $\tau_{x}$ to denote the translation of $x$ in $\mathbb{R}^{2}$ (i.e. $\tau_{x} y=y+x$ for all $\left.y \in \mathbb{R}^{2}\right)$. We will also denote $\Phi_{i}=\Phi \backslash\left\{\left(x_{i}, m_{i}\right)\right\}$ for $i \in I, \Phi_{i j}=\Phi \backslash\left\{\left(x_{i}, m_{i}\right),\left(x_{j}, m_{j}\right)\right\}$ for $i \neq j$ in $I$, and their associated shot noise random fields

$$
\forall i, \quad X_{\Phi_{i}}(x)=\sum_{k ; k \neq i} g_{m_{k}}\left(x-x_{k}\right) \quad \text { and } \quad \forall j \neq i, \quad X_{\Phi_{i j}}(x)=\sum_{k ; k \neq i, k \neq j} g_{m_{k}}\left(x-x_{k}\right) .
$$

Theorem 4. Assume that for F-almost every $m \in \mathbb{R}^{d}$, the function $g_{m}$ is an elementary function on $\mathbb{R}^{2}$ (Definition 5) satisfying (11), (21) and (22), and such that

$$
\begin{aligned}
& \int_{\mathbb{R}^{d}} \mathcal{H}^{0}\left(\mathcal{S}_{g_{m}} \backslash \mathcal{R}_{g_{m}}\right) F(d m)<+\infty \\
& \int_{\mathbb{R}^{d} \times \mathbb{R}^{d}} \int_{\mathbb{R}^{2}} \mathcal{H}^{0}\left(\mathcal{R}_{g_{m^{\prime}}} \cap \tau_{x} \mathcal{R}_{g_{m}}\right) d x F(d m) F\left(d m^{\prime}\right)<+\infty \\
& \int_{\mathbb{R}^{d} \times \mathbb{R}^{d}} \int_{\mathbb{R}^{2}} \mathcal{H}^{0}\left(\left\{y \in \mathcal{R}_{g_{m^{\prime}}} \cap \tau_{x} \mathcal{R}_{g_{m}} ; \nu_{g_{m^{\prime}}}(y)= \pm \nu_{g_{m}}(y-x)\right\}\right) d x F(d m) F\left(d m^{\prime}\right)=0 .
\end{aligned}
$$

Then, almost surely, for all bounded open set $U \subset \mathbb{R}^{2}, X_{\Phi}$ is an elementary function on $U$ and its discontinuity set on $U$ is given by $\mathcal{S}_{X_{\Phi}} \cap U$ where $\mathcal{S}_{X_{\Phi}}=\cup_{i} \tau_{x_{i}} g_{m_{i}}$ may be written as $\mathcal{S}_{X_{\Phi}}=\mathcal{R}_{X_{\Phi}} \cup \mathcal{C}_{X_{\Phi}} \cup \mathcal{I}_{X_{\Phi}}$, with

- $\mathcal{R}_{X_{\Phi}}=\left(\bigcup_{i} \tau_{x_{i}} \mathcal{R}_{g_{m_{i}}}\right) \backslash\left(\bigcup_{i, j \neq} \tau_{x_{i}} \mathcal{R}_{g_{m_{i}}} \cap \tau_{x_{j}} \mathcal{R}_{g_{m_{j}}}\right)$, and if $x \in \mathcal{R}_{X_{\Phi}} \cap U$, there exists a unique $i$ such that $x \in \tau_{x_{i}} \mathcal{R}_{g_{m_{i}}}$.

- $\mathcal{C}_{X_{\Phi}}=\bigcup_{i} \tau_{x_{i}} \mathcal{C}_{g_{m_{i}}}$, and if $x \in \mathcal{C}_{X_{\Phi}} \cap U$, there exists a unique $i$ such that $x \in \tau_{x_{i}} \mathcal{C}_{g_{m_{i}}}$. - $\mathcal{I}_{X_{\Phi}}=\left(\bigcup_{i} \tau_{x_{i}} \mathcal{I}_{g_{m_{i}}}\right) \cup\left(\bigcup_{i, j \neq} \tau_{x_{i}} \mathcal{R}_{g_{m_{i}}} \cap \tau_{x_{j}} \mathcal{R}_{g_{m_{j}}}\right)$ and if $x \in \mathcal{I}_{X_{\Phi}} \cap U$, only two situations
occur

- there exists a unique $i$ such that $x \in \tau_{x_{i}} \mathcal{I}_{g_{m_{i}}}$.

- or there exists a unique pair $\{i, j\}$ with $i \neq j$ such that $x \in \tau_{x_{i}} \mathcal{R}_{g_{m_{i}}} \cap \tau_{x_{j}} \mathcal{R}_{g_{m_{j}}}$. 
In particular, a.s.

$$
V_{X_{\Phi}}(U)=\sum_{i} V_{g_{m_{i}}}\left(\tau_{-x_{i}} U\right) \text { and } \operatorname{LTC}_{X_{\Phi}}(U)=\sum_{i} \operatorname{LTC}_{g_{m_{i}}}\left(\tau_{-x_{i}} U\right) .
$$

Proof. Since it is sufficient to prove the result for all rectangles $U=\left(a_{1}, b_{1}\right) \times\left(a_{2}, b_{2}\right)$ with $a_{1}, a_{2}, b_{1}, b_{2} \in \mathbb{Q}$, we only have to prove it holds almost surely on some fixed rectangle $U=$ $\left(a_{1}, b_{1}\right) \times\left(a_{2}, b_{2}\right)$, with $a_{1}<b_{1}$ and $a_{2}<b_{2}$. Let us notice that we already have proven that $X_{\Phi} \in S B V(U)$ in Theorem 2 of [11] in a more general framework. However we need here to be more precise on its discontinuity set $\mathcal{S}_{X_{\Phi}} \subset \cup_{i} \tau_{x_{i}} \mathcal{S}_{g_{m_{i}}}$.

Let us first remark that when $A_{\Phi}$ is a finite set of points of $\mathbb{R}^{2}$ depending on the marked Poisson point process $\Phi=\left\{\left(x_{i}, m_{i}\right)\right\}$, as soon as $\mathbb{E}\left(\mathcal{H}^{0}\left(A_{\Phi}\right)\right)<+\infty$, one has,

$$
\bigcup_{j} A_{\Phi_{j}} \cap \tau_{x_{j}} \mathcal{S}_{g_{m_{j}}}=\emptyset \quad \text { a.s.. }
$$

This follows from the fact that, by Slivnyak-Mecke formula (see [8] Theorem 1.4.5),

$$
\begin{aligned}
\mathbb{E}\left(\mathcal{H}^{0}\left(\bigcup_{j} A_{\Phi_{j}} \cap \tau_{x_{j}} \mathcal{S}_{g_{m_{j}}}\right)\right) & \leq \lambda \int_{\mathbb{R}^{2} \times \mathbb{R}^{d}} \mathbb{E}\left(\mathcal{H}^{0}\left(A_{\Phi} \cap \tau_{x} \mathcal{S}_{g_{m}}\right)\right) d x F(d m) \\
& \leq \lambda \mathbb{E}\left(\mathcal{H}^{0}\left(A_{\Phi}\right)\right) \int_{\mathbb{R}^{d}} \mathcal{L}\left(\mathcal{S}_{g_{m}}\right) F(d m)=0,
\end{aligned}
$$

since $\mathcal{L}\left(\mathcal{S}_{g_{m}}\right)=0$, using Fubini Theorem and the translation invariance of both $\mathcal{H}^{0}$ and $\mathcal{L}$.

Our first assumption (24) implies that

$$
\bigcup_{i, j \neq} \tau_{x_{i}}\left(\mathcal{S}_{g_{m_{i}}} \backslash \mathcal{R}_{g_{m_{i}}}\right) \cap \tau_{x_{j}} \mathcal{S}_{g_{m_{j}}} \cap U=\emptyset \quad \text { a.s. }
$$

and as a consequence we have $\bigcup_{i, j \neq} \tau_{x_{i}} \mathcal{S}_{g_{m_{i}}} \cap \tau_{x_{j}} \mathcal{S}_{g_{m_{j}}} \cap U=\bigcup_{i, j \neq} \tau_{x_{i}} \mathcal{R}_{g_{m_{i}}} \cap \tau_{x_{j}} \mathcal{R}_{g_{m_{j}}} \cap U$ a.s.. Indeed, taking $A_{\Phi}=\bigcup_{i} \tau_{x_{i}}\left(\mathcal{S}_{g_{m_{i}}} \backslash \mathcal{R}_{g_{m_{i}}}\right) \cap U$, Campbell formula (see [8] Theorem 1.4.3) ensures that

$$
\begin{aligned}
\mathbb{E}\left(\mathcal{H}^{0}\left(A_{\Phi}\right)\right) & \leq \lambda \int_{\mathbb{R}^{2} \times \mathbb{R}^{d}} \mathcal{H}^{0}\left(\tau_{x}\left(\mathcal{S}_{g_{m}} \backslash \mathcal{R}_{g_{m}}\right) \cap U\right) d x F(d m) \\
& \leq \lambda \mathcal{L}(U) \int_{\mathbb{R}^{d}} \mathcal{H}^{0}\left(\mathcal{S}_{g_{m}} \backslash \mathcal{R}_{g_{m}}\right) F(d m)<+\infty .
\end{aligned}
$$

Then, (27) follows from the preceding remark since

$$
\bigcup_{i, j \neq} \tau_{x_{i}}\left(\mathcal{S}_{g_{m_{i}}} \backslash \mathcal{R}_{g_{m_{i}}}\right) \cap \tau_{x_{j}} \mathcal{S}_{g_{m_{j}}} \cap U=\bigcup_{j} A_{\Phi_{j}} \cap \tau_{x_{j}} \mathcal{S}_{g_{m_{j}}} .
$$

The second assumption (25) will ensure both that

$$
\mathcal{H}^{0}\left(\bigcup_{i, j \neq} \tau_{x_{i}} \mathcal{R}_{g_{m_{i}}} \cap \tau_{x_{j}} \mathcal{R}_{g_{m_{j}}} \cap U\right)<\infty \quad \text { a.s. }
$$

and

$$
\bigcup_{i, j, k \neq} \tau_{x_{i}} \mathcal{R}_{g_{m_{i}}} \cap \tau_{x_{j}} \mathcal{R}_{g_{m_{j}}} \cap \tau_{x_{k}} \mathcal{S}_{g_{m_{k}}} \cap U=\emptyset \quad \text { a.s.. }
$$


Here we set $A_{\Phi}=\bigcup_{i, j \neq} \tau_{x_{i}} \mathcal{R}_{g_{m_{i}}} \cap \tau_{x_{j}} \mathcal{R}_{g_{m_{j}}} \cap U$. Using again Slivnyak-Mecke formula and Campbell formula we obtain that

$$
\begin{aligned}
\mathbb{E}\left(\mathcal{H}^{0}\left(A_{\Phi}\right)\right) & \leq \lambda^{2} \int_{\mathbb{R}^{2} \times \mathbb{R}^{d}} \int_{\mathbb{R}^{2} \times \mathbb{R}^{d}} \mathcal{H}^{0}\left(\tau_{x} \mathcal{R}_{g_{m}} \cap \tau_{x^{\prime}} \mathcal{R}_{g_{m^{\prime}}} \cap U\right) d x F(d m) d x^{\prime} F\left(d m^{\prime}\right) \\
& \leq \lambda^{2} \mathcal{L}(U) \int_{\mathbb{R}^{d} \times \mathbb{R}^{d}} \int_{\mathbb{R}^{2}} \mathcal{H}^{0}\left(\mathcal{R}_{g_{m^{\prime}}} \cap \tau_{x} \mathcal{R}_{g_{m}}\right) d x F(d m) F\left(d m^{\prime}\right)<+\infty
\end{aligned}
$$

It follows that $\mathcal{H}^{0}\left(A_{\Phi}\right)<+\infty$ a.s. and $\bigcup_{k} A_{\Phi_{k}} \cap \tau_{x_{k}} \mathcal{S}_{g_{m_{k}}}=\emptyset$ a.s..

Finally and similarly, the last assumption ensures that

$$
\left\{y \in \bigcup_{i, j \neq} \tau_{x_{i}} \mathcal{R}_{g_{m_{i}}} \cap \tau_{x_{j}} \mathcal{R}_{g_{m_{j}}} \cap U ; \nu_{g_{m_{i}}}\left(y-x_{i}\right)= \pm \nu_{g_{m_{j}}}\left(y-x_{j}\right)\right\}=\emptyset \quad \text { a.s.. }
$$

This follows from the fact that the expected $\mathcal{H}^{0}$ measure of this set is zero, according to SlivnyakMecke formula and Campbell formula, Fubini Theorem and translation invariance.

Now, let us remark that $X_{\Phi}$ coincides on $U$ with $X_{\tilde{\Phi}}$ where $\tilde{\Phi}=\left\{\left(x_{i}, m_{i}\right) \in \Phi ; \tau_{x_{i}}\left[-T_{m_{i}}, T_{m_{i}}\right] \cap\right.$ $U \neq \emptyset\}$ and $N(U)=\# \tilde{\Phi}$ is a.s. finite, as a consequence of (23) under the assumption that $\int_{\mathbb{R}^{d}} T_{m}^{2} F(d m)<+\infty$. Moreover $X_{\tilde{\Phi}}$ is an elementary function (as a finite sum of elementary functions in generic position thanks to (28), (29) and (30)) and therefore $X_{\Phi}$ is an elementary function on $U$.

Theorem 5. Under the hypothesis of Theorem 4, assuming moreover that

$$
\int_{\mathbb{R}^{d} \times \mathbb{R}^{d}} \int_{\mathbb{R}^{2}}\left(\left\|g_{m}\right\|_{\infty}+\left\|g_{m^{\prime}}\right\|_{\infty}\right) \mathcal{H}^{0}\left(\mathcal{R}_{g_{m^{\prime}}} \cap \tau_{x} \mathcal{R}_{g_{m}}\right) d x F(d m) F\left(d m^{\prime}\right)<+\infty
$$

then the random variables $V_{X_{\Phi}}(U), \operatorname{LTaC}_{X_{\Phi}}(U)$ and $\operatorname{LTC}_{X_{\Phi}}(U)$ have finite expectation for any bounded open set $U$. Moreover one has

$$
\mathbb{E}\left(V_{X_{\Phi}}(U)\right)=\lambda \mathcal{L}(U) \int_{\mathbb{R}^{d}} V_{g_{m}}\left(\mathbb{R}^{2}\right) F(d m) \text { and } \mathbb{E}\left(\operatorname{LTC}_{X_{\Phi}}(U)\right)=\lambda \mathcal{L}(U) \int_{\mathbb{R}^{d}} \operatorname{LTC}_{g_{m}}\left(\mathbb{R}^{2}\right) F(d m) .
$$

It follows that for a.e. $t \in \mathbb{R}$, the random variables $\operatorname{Per}\left(E_{X_{\Phi}}(t), U\right)$ and $\operatorname{TC}\left(\partial E_{X_{\Phi}}(t), U\right)$ have also finite expectation such that for any $h$ continuous bounded function, denoting by $\bar{h}_{X_{\Phi}(0)}$ the function $s \mapsto \mathbb{E}\left(h\left(X_{\Phi}(0)+s\right)\right)$,

$$
\mathbb{E}\left(\operatorname{LP}_{X_{\Phi}}(h, U)\right)=\int_{\mathbb{R}} h(t) \mathbb{E}\left(\operatorname{Per}\left(E_{X_{\Phi}}(t), U\right)\right) d t=\lambda \mathcal{L}(U) \overline{\operatorname{LP}_{X_{\Phi}}}(h),
$$

and

$$
\mathbb{E}\left(\operatorname{LTC}_{X_{\Phi}}(h, U)\right)=\int_{\mathbb{R}} h(t) \mathbb{E}\left(\operatorname{TC}\left(\partial E_{X_{\Phi}}(t), U\right)\right) d t=\lambda \mathcal{L}(U) \overline{\operatorname{LTC}_{X_{\Phi}}}(h),
$$

where

$$
\begin{aligned}
\overline{\operatorname{LP}_{X_{\Phi}}}(h) & =\int_{\mathbb{R}^{d}} \operatorname{LP}_{g_{m}}\left(\bar{h}_{X_{\Phi}(0)}, \mathbb{R}^{2}\right) F(d m) \\
\overline{\operatorname{LTC}_{X_{\Phi}}}(h) & =\lambda \int_{\mathbb{R}^{d}}\left(\operatorname{LTC}_{g_{m}}\left(\bar{h}_{X_{\Phi}(0)}, \mathbb{R}^{2}\right)+\lambda I\left(\bar{h}_{X_{\Phi}(0)}, m\right)\right) F(d m),
\end{aligned}
$$


with

$$
\begin{aligned}
I\left(\bar{h}_{X_{\Phi}(0)}, m\right)=\frac{1}{2} & \int_{\mathbb{R}^{d}} \int_{\mathbb{R}^{2}} \sum_{z \in \tau_{x}} \sum_{\mathcal{R}_{g_{m}} \cap \mathcal{R}_{g_{m^{\prime}}}} d_{S^{1}}\left(\nu_{g_{m}}(z-x), \nu_{g_{m^{\prime}}}(z)\right) \\
& \times \int_{g_{m^{\prime}}^{-}(z)}^{g_{m^{\prime}}^{+}(z)}\left(\bar{h}_{X_{\Phi}(0)}\left(s+g_{m}^{+}(z-x)\right)-\bar{h}_{X_{\Phi}(0)}\left(s+g_{m}^{-}(z-x)\right)\right) d s d x F\left(d m^{\prime}\right) .
\end{aligned}
$$

Proof. First note that the results on the perimeter follow from Theorem 2 and Proposition 1 of [11] since in view of (11) and (21) we have $\int_{\mathbb{R}^{d}}\left\|g_{m}\right\|_{B V\left(\mathbb{R}^{d}\right)} F(d m)<+\infty$. Actually, it yields that

$$
\begin{aligned}
\mathbb{E}\left(\operatorname{LP}_{X_{\Phi}}(h, U)\right) & =\lambda \mathcal{L}(U) \int_{\mathbb{R}^{d}} \int_{\mathcal{R}_{g_{m}}}\left(\int_{g_{m}^{-}(z)}^{g_{m}^{+}(z)} \mathbb{E}\left(h\left(s+X_{\Phi}(0)\right) d s\right) \mathcal{H}^{1}(d z) F(d m)\right. \\
& =\lambda \mathcal{L}(U) \int_{\mathbb{R}^{d}} \int_{\mathcal{R}_{g_{m}}}\left(\bar{H}_{X_{\Phi}(0)}\left(g_{m}^{+}(z)\right)-\bar{H}_{X_{\Phi}(0)}\left(g_{m}^{-}(z)\right)\right) \mathcal{H}^{1}(d z) F(d m) \\
& =\lambda \mathcal{L}(U) \int_{\mathbb{R}^{d}} \operatorname{LP}_{g_{m}}\left(\bar{h}_{X_{\Phi}(0)}, \mathbb{R}^{2}\right) F(d m),
\end{aligned}
$$

where we introduced $\bar{H}_{X_{\Phi}(0)}$ to denote a primitive of the function $\bar{h}_{X_{\Phi}(0)}$.

Moreover, according to Proposition 4, one has a.s.

$\operatorname{LTaC}_{X_{\Phi}}(U) \leq \sum_{i} \operatorname{LTaC}_{g_{m_{i}}}\left(\tau_{-x_{i}} U\right)+2 \pi \sum_{i, j \neq} \mathcal{H}^{0}\left(\tau_{x_{i}} \mathcal{R}_{g_{m_{i}}} \cap \tau_{x_{j}} \mathcal{R}_{g_{m_{j}}} \cap U\right)\left(\left\|g_{m_{i}}\right\|_{\infty}+\left\|g_{m_{j}}\right\|_{\infty}\right)$.

By Campbell formula,

$$
\mathbb{E}\left(\sum_{i} \operatorname{LTaC}_{g_{m_{i}}}\left(\tau_{-x_{i}} U\right)\right)=\int_{\mathbb{R}^{2} \times \mathbb{R}^{d}} \operatorname{LTaC}_{g_{m}}\left(\tau_{-x} U\right) \lambda d x F(d m) .
$$

Hence, by Fubini Theorem,

$$
\mathbb{E}\left(\sum_{i} \operatorname{LTaC}_{g_{m_{i}}}\left(\tau_{-x_{i}} U\right)\right)=\lambda \mathcal{L}(U) \int_{\mathbb{R}^{d}} \operatorname{LTaC}_{g_{m}}\left(\mathbb{R}^{2}\right) F(d m)<+\infty
$$

Moreover, by Slivnyak-Mecke formula,

$$
\begin{aligned}
& \mathbb{E}\left(\sum_{i, j \neq} \mathcal{H}^{0}\left(\tau_{x_{i}} \mathcal{R}_{g_{m_{i}}} \cap \tau_{x_{j}} \mathcal{R}_{g_{m_{j}}} \cap U\right)\left(\left\|g_{m_{i}}\right\|_{\infty}+\left\|g_{m_{j}}\right\|_{\infty}\right)\right) \\
= & \int_{\mathbb{R}^{d} \times \mathbb{R}^{d}} \int_{\mathbb{R}^{2} \times \mathbb{R}^{2}} \mathcal{H}^{0}\left(\tau_{x} \mathcal{R}_{g_{m}} \cap \tau_{x^{\prime}} \mathcal{R}_{g_{m^{\prime}}} \cap U\right)\left(\left\|g_{m}\right\|_{\infty}+\left\|g_{m^{\prime}}\right\|_{\infty}\right) \lambda^{2} d x d x^{\prime} F(d m) F\left(d m^{\prime}\right) \\
= & \lambda^{2} \mathcal{L}(U) \int_{\mathbb{R}^{d} \times \mathbb{R}^{d}} \int_{\mathbb{R}^{2}} \int_{\mathbb{R}^{2}} \mathbb{I}_{z \in \mathcal{R}_{g_{m^{\prime}}} \cap \tau_{\tau_{x}} \mathcal{R}_{g_{m}}}\left(\left\|g_{m}\right\|_{\infty}+\left\|g_{m^{\prime}}\right\|_{\infty}\right) \mathcal{H}^{0}(d z) d x F(d m) F\left(d m^{\prime}\right) \\
= & \lambda^{2} \mathcal{L}(U) \int_{\mathbb{R}^{d} \times \mathbb{R}^{d}} \int_{\mathbb{R}^{2}}\left(\left\|g_{m}\right\|_{\infty}+\left\|g_{m^{\prime}}\right\|_{\infty}\right) \mathcal{H}^{0}\left(\mathcal{R}_{g_{m^{\prime}}} \cap \tau_{x} \mathcal{R}_{g_{m}}\right) d x F(d m) F\left(d m^{\prime}\right)<+\infty,
\end{aligned}
$$

by assumption (31). It follows that $\mathbb{E}\left(\operatorname{LTaC}_{X_{\Phi}}(U)\right)<+\infty$ and therefore

$$
\mathbb{E}\left(\int_{\mathbb{R}}\left|\mathrm{TC}\left(\partial E_{X_{\Phi}}(t), U\right)\right| d t\right)<+\infty .
$$


So, for a.e. $t \in \mathbb{R}$, the random variable $\mathrm{TC}\left(\partial E_{X_{\Phi}}(t), U\right)$ has a finite expectation and $t \mapsto$ $\mathbb{E}\left(\operatorname{TC}\left(\partial E_{X_{\Phi}}(t), U\right)\right)$ is a function in $L^{1}(\mathbb{R})$. Note that since $\operatorname{LTC}_{X_{\Phi}}(U)=\sum_{i} \operatorname{LTC}_{g_{m_{i}}}\left(\tau_{-x_{i}} U\right)$ we simply get by Campbell formula and Fubini Theorem that

$$
\mathbb{E}\left(\operatorname{LTC}_{X_{\Phi}}(U)\right)=\lambda \mathcal{L}(U) \int_{\mathbb{R}^{d}} \operatorname{LTC}_{g_{m}}\left(\mathbb{R}^{2}\right) F(d m) .
$$

Now, let $h$ be a continuous bounded function with primitive denoted by $H$. We already know that a.s. $\operatorname{LTC}_{X_{\Phi}}(h, U)=\int_{\mathbb{R}} h(t) \mathrm{TC}\left(\partial E_{X_{\Phi}}(t), U\right) d t$ may be written as the sum of three terms $R_{h}+C_{h}+I_{h}$ with finite expectation under our assumptions. By Fubini Theorem, it follows that

$$
\mathbb{E}\left(\operatorname{LTC}_{X_{\Phi}}(h, U)\right)=\int_{\mathbb{R}} h(t) \mathbb{E}\left(\operatorname{TC}\left(\partial E_{X_{\Phi}}(t), U\right)\right) d t=\mathbb{E}\left(R_{h}\right)+\mathbb{E}\left(C_{h}\right)+\mathbb{E}\left(I_{h}\right) .
$$

For the first term we have

$$
\begin{aligned}
& \mathbb{E}\left(R_{h}\right)=\mathbb{E}\left(\int_{\mathcal{R}_{X_{\Phi}} \cap U}\left[H\left(X_{\Phi}^{+}(z)\right)-H\left(X_{\Phi}^{-}(x)\right)\right] \kappa_{X_{\Phi}}(z) \mathcal{H}^{1}(d z)\right) \\
= & \mathbb{E}\left(\sum_{i} \int_{\tau_{x_{i}} \mathcal{R}_{g_{m_{i}}} \cap U}\left[H\left(X_{\Phi_{i}}(z)+g_{m_{i}}^{+}\left(z-x_{i}\right)\right)-H\left(X_{\Phi_{i}}(z)+g_{m_{i}}^{-}\left(z-x_{i}\right)\right)\right] \kappa_{g_{m_{i}}}\left(z-x_{i}\right) \mathcal{H}^{1}(d z)\right) \\
= & \int_{\mathbb{R}^{2} \times \mathbb{R}^{d}} \int_{\tau_{x} \mathcal{R}_{g_{m}} \cap U} \mathbb{E}\left(\left[H\left(X_{\Phi}(z)+g_{m}^{+}(z-x)\right)-H\left(X_{\Phi}(z)+g_{m}^{-}(z-x)\right)\right]\right) \kappa_{g_{m}}(z-x) \mathcal{H}^{1}(d z) \lambda d x F(d m),
\end{aligned}
$$

by Slivnyak-Mecke formula. Using the translation invariance of both $\mathcal{H}^{1}$ and $\mathcal{L}$ and the stationarity of $X_{\Phi}$, we get that

$$
\begin{aligned}
\mathbb{E}\left(R_{h}\right) & =\lambda \mathcal{L}(U) \int_{\mathbb{R}^{d}} \int_{\mathcal{R}_{g_{m}}} \int_{g_{m}^{-}(z)}^{g_{m}^{+}(z)} \mathbb{E}\left(h\left(X_{\Phi}(0)+s\right)\right) \kappa_{g_{m}}(z) d s \mathcal{H}^{1}(d z) F(d m) \\
& =\lambda \mathcal{L}(U) \int_{\mathbb{R}^{d}} \int_{\mathcal{R}_{g_{m}}}\left(\bar{H}_{X_{\Phi}(0)}\left(g_{m}^{+}(z)\right)-\bar{H}_{X_{\Phi}(0)}\left(g_{m}^{-}(z)\right)\right) \kappa_{g_{m}}(z) \mathcal{H}^{1}(d z) F(d m) .
\end{aligned}
$$

Similarly, for the second term we have

$$
\begin{gathered}
\mathbb{E}\left(C_{h}\right)=\mathbb{E}\left(\sum_{z \in \mathcal{C}_{X_{\Phi}} \cap U}\left[H\left(X_{\Phi}^{+}(z)\right)-H\left(X_{\Phi}^{-}(z)\right)\right] \alpha_{X_{\Phi}}(z)\right) \\
=\mathbb{E}\left(\sum_{i} \mathbb{I}_{z \in \tau_{x_{i}} \mathcal{C}_{g_{m_{i}}} \cap U}\left[H\left(X_{\Phi_{i}}(z)+g_{m_{i}}^{+}\left(z-x_{i}\right)\right)-H\left(X_{\Phi_{i}}(z)+g_{m_{i}}^{-}\left(z-x_{i}\right)\right)\right] \alpha_{g_{m_{i}}}\left(z-x_{i}\right) \mathcal{H}^{1}(d z)\right) \\
=\lambda \mathcal{L}(U) \int_{\mathbb{R}^{d}} \sum_{z \in \mathcal{C}_{g_{m}}} \mathbb{E}\left(\left[H\left(X_{\Phi}(0)+g_{m}^{+}(z)\right)-H\left(X_{\Phi}(0)+g_{m}^{-}(z)\right)\right]\right) \alpha_{g_{m}}(z) F(d m) \\
=\lambda \mathcal{L}(U) \int_{\mathbb{R}^{d}} \sum_{z \in \mathcal{C}_{g_{m}}}\left(\bar{H}_{X_{\Phi}(0)}\left(g_{m}^{+}(z)\right)-\bar{H}_{X_{\Phi}(0)}\left(g_{m}^{-}(z)\right)\right) \alpha_{g_{m}}(z) F(d m) .
\end{gathered}
$$

Finally, the last term may be itself decomposed in two terms, say $I_{h}=I_{h}^{(1)}+I_{h}^{(2)}$. With similar 
computations we get that $\mathbb{E}\left(I_{h}^{(1)}\right)$ is equal to

$$
\begin{aligned}
& \lambda \mathcal{L}(U) \int_{\mathbb{R}^{d}} \sum_{z \in \mathcal{I}_{g_{m}}}\left(\int_{g_{m_{-}^{+}}(z)}^{g_{m}^{+}(z)} \mathbb{E}\left(h\left(X_{\Phi}(0)+s\right)\right) d s-\int_{g_{m}^{-}(z)}^{g_{m_{+}^{-}}^{-}(z)} \mathbb{E}\left(h\left(X_{\Phi}(0)+s\right)\right) d s\right) \beta_{g_{m}}(z) F(d m) \\
= & \lambda \mathcal{L}(U) \int_{\mathbb{R}^{d}} \sum_{z \in \mathcal{I}_{g_{m}}}\left[\bar{H}_{X_{\Phi}(0)}\left(g_{m}^{+}(z)\right)+\bar{H}_{X_{\Phi}(0)}\left(g_{m}^{-}(z)\right)-\bar{H}_{X_{\Phi}(0)}\left(g_{m_{-}}^{+}(z)\right)-\bar{H}_{X_{\Phi}(0)}\left(g_{m_{+}}(z)\right)\right] F(d m) .
\end{aligned}
$$

Adding (32),(33), and (34) we recognize

$$
\lambda \mathcal{L}(U) \int_{\mathbb{R}^{d}} \operatorname{LTC}_{g_{m}}\left(\bar{h}_{X_{\Phi}(0)}, \mathbb{R}^{2}\right) F(d m) .
$$

For the remaining term, let us introduce

$$
\Delta_{m_{i}, m_{j}} h\left(t, z-x_{i}, z-x_{j}\right)=\int_{g_{m_{j}}^{-}\left(z-x_{j}\right)}^{g_{m_{j}}^{+}\left(z-x_{j}\right)}\left[h\left(t+g_{m_{i}}^{+}\left(z-x_{i}\right)+s\right)-h\left(t+g_{m_{i}}^{-}\left(z-x_{i}\right)+s\right)\right] d s
$$

for $z \in \tau_{x_{i}} \mathcal{R}_{g_{m_{i}}} \cap \tau_{x_{j}} \mathcal{R}_{g_{m_{j}}}$. Since $\tau_{x_{i}} \mathcal{R}_{g_{m_{i}}} \cap \tau_{x_{j}} \mathcal{R}_{g_{m_{j}}}=\tau_{x_{j}} \mathcal{R}_{g_{m_{j}}} \cap \tau_{x_{i}} \mathcal{R}_{g_{m_{i}}}$, the term $\mathbb{E}\left(I_{h}^{(2)}\right)$ is equal to

$$
\begin{gathered}
\mathbb{E}\left(\frac{1}{2} \sum_{i \neq j} \sum_{\left.z \in \tau_{x_{i}} \mathcal{R}_{g_{m_{i}} \cap \tau_{x_{j}}} \mathcal{R}_{g_{m_{j}} \cap U} \Delta_{m_{i}, m_{j}} h\left(X_{\Phi_{i j}}(z), z-x_{i}, z-x_{j}\right) d_{S^{1}}\left(\nu_{g_{m_{i}}}\left(z-x_{i}\right), \nu_{g_{m_{j}}}\left(z-x_{j}\right)\right)\right)}=\frac{1}{2} \iint_{z \in \tau_{x} \mathcal{R}_{g_{m}} \cap \tau_{x^{\prime}} \mathcal{R}_{g_{m^{\prime}}} \cap U} \mathbb{E}\left(\Delta_{m, m^{\prime}} h\left(X_{\Phi}(z), z-x, z-x^{\prime}\right)\right)\right. \\
\quad \times d_{S^{1}}\left(\nu_{g_{m}}(z-x), \nu_{g_{m^{\prime}}}\left(z-x^{\prime}\right)\right) \lambda^{2} d x F(d m) d x^{\prime} F\left(d m^{\prime}\right),
\end{gathered}
$$

by Slivnyak-Mecke formula. By change of variables, translation invariance of $\mathcal{H}^{0}$ and $\mathcal{L}$, and stationarity of $X_{\Phi}$, we get that $\mathbb{E}\left(I_{h}^{(2)}\right)$ is equal to

$$
\begin{gathered}
\frac{\lambda^{2}}{2} \mathcal{L}(U) \int_{\mathbb{R}^{d} \times \mathbb{R}^{d}} \int_{\mathbb{R}^{2}} \sum_{z \in \tau_{x} \mathcal{R}_{g_{m}} \cap \mathcal{R}_{g_{m^{\prime}}}} \mathbb{E}\left(\Delta_{m, m^{\prime}} \bar{h}_{X_{\Phi}(0)}(0, z-x, z)\right) d_{S^{1}}\left(\nu_{g_{m}}(z-x), \nu_{g_{m^{\prime}}}(z)\right) d x F(d m) F\left(d m^{\prime}\right) \\
=\lambda^{2} \mathcal{L}(U) \int_{\mathbb{R}^{d}} I\left(\bar{h}_{X_{\Phi}(0)}, m\right)
\end{gathered}
$$

Remark: Let us notice that choosing $h_{u}(s)=e^{i u s}$ for $u, s \in \mathbb{R}$ we have $\bar{h}_{u, X_{\Phi}(0)}=\mathbb{E}\left(e^{i u X_{\Phi}(0)}\right) \times$ $h_{u}$ and by linearity

$$
\begin{aligned}
& \operatorname{LP}_{g_{m}}\left(\bar{h}_{u, X_{\Phi}(0)}, \mathbb{R}^{2}\right)=\mathbb{E}\left(e^{i u X_{\Phi}(0)}\right) \operatorname{LP}_{g_{m}}\left(h_{u}, \mathbb{R}^{2}\right) \\
& \text { and } \operatorname{LTC}_{g_{m}}\left(\bar{h}_{u, X_{\Phi}(0)}, \mathbb{R}^{2}\right)=\mathbb{E}\left(e^{i u X_{\Phi}(0)}\right) \operatorname{LTC}_{g_{m}}\left(h_{u}, \mathbb{R}^{2}\right) .
\end{aligned}
$$


It follows that Fourier transforms of $t \mapsto \overline{\operatorname{Per}\left(E_{X}(t)\right)}$ and $t \mapsto \overline{\mathrm{TC}\left(\partial E_{X}(t)\right)}$ are given for $u \in \mathbb{R}$ by

$$
\overline{\operatorname{LP}_{X_{\Phi}}}\left(h_{u}\right)=\lambda \mathbb{E}\left(e^{i u X_{\Phi}(0)}\right) \int_{\mathbb{R}^{d}} \operatorname{LP}_{g_{m}}\left(h_{u}, \mathbb{R}^{2}\right) F(d m),
$$

and

$$
\begin{aligned}
& \overline{\operatorname{LP}_{X_{\Phi}}}\left(h_{u}\right)=\lambda \mathbb{E}\left(e^{i u X_{\Phi}(0)}\right) \int_{\mathbb{R}^{d}}\left[\operatorname{LTC}_{g_{m}}\left(h_{u}, \mathbb{R}^{2}\right)+\right. \\
& \left.\frac{\lambda}{2} \int_{\mathbb{R}^{d}} \int_{\mathbb{R}^{2}} \sum_{z \in \tau_{x} \mathcal{R}_{g_{m}} \cap \mathcal{R}_{g_{m^{\prime}}}}\left(e^{i u g_{m^{\prime}}^{+}(z)}-e^{i u g_{m^{\prime}}^{-}(z)}\right) \int_{g_{m}^{-}(z-x)}^{g_{m}^{+}(z-x)} e^{i u s} d_{S^{1}}\left(\nu_{g_{m}}(z-x), \nu_{g_{m^{\prime}}}(z)\right) d s d x F\left(d m^{\prime}\right)\right] F(d m) .
\end{aligned}
$$

\subsection{Explicit computations}

In this section, we will give some explicit computations of the mean level total curvature function of elementary shot noise random fields. These results generalize the results of Decreusefond et al. [15] obtained for indicator functions of a square, and also the known results on the Boolean model (that correspond to the excursion set of level $t=1$ ). We will also show some numerical simulations.

Let us first recall, as already used in Theorem 3, that for shot noise random fields, the characteristic function of $X_{\Phi}(0)$ is explicit and given by

$$
\forall u \in \mathbb{R}, \quad \mathbb{E}\left(e^{i u X_{\Phi}(0)}\right)=\exp \left(\lambda \int_{\mathbb{R}^{2} \times \mathbb{R}^{d}}\left(e^{i u g_{m}(x)}-1\right) d x F(d m)\right) .
$$

We will consider here $D$ an elementary compact subset of $\mathbb{R}^{2}$. Its boundary $\Gamma=\partial D$ is a finite union of positively oriented closed simple curves, piecewise $C^{2}$ and of finite total curvature, i.e. $\operatorname{TaC}\left(\Gamma, \mathbb{R}^{2}\right)<+\infty$. Note that by Gauss-Bonnet Theorem we have

$$
\mathrm{TC}\left(\Gamma, \mathbb{R}^{2}\right)=2 \pi \chi(D)
$$

We will focus on the case where the marks are of the form $m=(b, r, \theta) \in \mathbb{R} \times[0,+\infty) \times[0,2 \pi] \subset \mathbb{R}^{d}$ with $d=3$, with distribution $F(d m)=F_{B}(d b) F_{R}(d r) F_{\Theta}(d \theta)$ and functions $g_{m}$ given by

$$
\forall x \in \mathbb{R}^{2}, \quad g_{m}(x)=b \mathbf{I}_{R_{\theta} r D}(x),
$$

where $r D$ is the dilation of $D$ by the factor $r$, and $R_{\theta}$ denotes the rotation of angle $\theta$. We will denote $b^{+}=\max (b, 0), b^{-}=\min (0, b)$ such that for $x \in \partial R_{\theta} r D$, we have $g_{m}^{+}(x)=b^{+}$and $g_{m^{-}}(x)=b^{-}$with $g_{m}^{+}(x)-g_{m^{-}}(x)=b^{+}-b^{-}=|b|$ and $b=\left(b^{+}-b^{-}\right) \operatorname{sgn}(b)$.

We denote by $B\left(\right.$ resp. $B^{+}=\max (B, 0)$ and $\left.B^{-}=\min (B, 0)\right), R$ and $\Theta$ independent random variables with distributions $F_{B}, F_{R}$ and $F_{\Theta}$. We will mainly focus on the case where $\Theta$ is uniform on $[0,2 \pi]$, that is $F_{\Theta}(d \theta)=\frac{1}{2 \pi} \mathbb{I}_{[0,2 \pi]} d \theta$ for random shapes with uniform rotation; or on the case where $\Theta=0$ a.s., that is $F_{\Theta}=\delta_{0}$ corresponding to simpler marks $m=(b, r)$.

Theorem 6. We assume that

$$
\mathbb{E}(|B|)<+\infty \quad \text { and } \quad \mathbb{E}\left(R^{2}\right)<+\infty .
$$


We denote the mean perimeter and the mean area of $R_{\theta} r D$ by

$$
\begin{aligned}
& \bar{p}:=\int_{\mathbb{R}^{2}} \operatorname{Per}\left(R_{\theta} r D\right) F_{R}(d r) F_{\Theta}(d \theta)=\operatorname{Per}(D) \mathbb{E}(R) \\
& \text { and } \quad \bar{a}:=\int_{\mathbb{R}^{2}} \mathcal{L}\left(R_{\theta} r D\right) F_{R}(d r) F_{\Theta}(d \theta)=\mathcal{L}(D) \mathbb{E}\left(R^{2}\right) .
\end{aligned}
$$

Then $X_{\Phi}$ satisfies the assumptions of Theorem 4 and 5 and

$$
\mathbb{E}\left(\operatorname{LP}_{X_{\Phi}}(U)\right)=\lambda \mathcal{L}(U) \mathbb{E}(|B|) \bar{p} \quad \text { and } \quad \mathbb{E}\left(\operatorname{LTC}_{X_{\Phi}}(U)\right)=2 \pi \lambda \mathcal{L}(U) \mathbb{E}(B) \chi(D) .
$$

Moreover, when $F_{\Theta}$ is the uniform law on $[0,2 \pi]$, then, for all $u \neq 0$,

$$
\begin{aligned}
\int_{\mathbb{R}} e^{i u t} \overline{\operatorname{Per}\left(E_{X_{\Phi}}(t)\right)} d t= & \lambda \mathbb{E}\left(e^{i u X_{\Phi}(0)}\right) \frac{\widehat{F_{B^{+}}}(u)-\widehat{F_{B^{-}}}(u)}{i u} \bar{p} \\
\int_{\mathbb{R}} e^{i u t} \overline{\operatorname{TC}\left(\partial E_{X_{\Phi}}(t)\right)} d t= & \lambda \mathbb{E}\left(e^{i u X_{\Phi}(0)}\right) \\
& \times \frac{1}{i u}\left(2 \pi\left(\widehat{F_{B}}(u)-1\right) \chi(D)+\frac{\lambda}{2}\left(\widehat{F_{B^{+}}}(u)-\widehat{F_{B^{-}}}(u)\right)^{2} \bar{p}^{2}\right),
\end{aligned}
$$

where $\widehat{F_{B}}$, respectively $\widehat{F_{B^{+}}}$or $\widehat{F_{B^{-}}}$, denotes the characteristic function of $B$, respectively $B^{+}$or $B^{-}$. In the case where $B=1\left(=B^{+}\right)$a.s. we deduce that $\forall k \in \mathbb{N}, \forall t \in(k, k+1]$,

$$
\begin{aligned}
\overline{\operatorname{Per}\left(E_{X_{\Phi}}(t)\right)}=\lambda e^{-\lambda \bar{a}} \frac{(\lambda \bar{a})^{k}}{k !} \bar{p} & \text { and } \\
& \quad \overline{\operatorname{TC}\left(\partial E_{X_{\Phi}}(t)\right)}=2 \pi \lambda e^{-\lambda \bar{a}} \frac{(\lambda \bar{a})^{k}}{k !}\left(\chi(D)-\frac{\lambda}{4 \pi} \bar{p}^{2}+\frac{\bar{p}^{2}}{4 \pi \bar{a}} k\right) .
\end{aligned}
$$

Proof. Since $\mathcal{H}^{1}\left(R_{\theta} r \Gamma\right)=r \mathcal{H}^{1}(\Gamma)$ and $\operatorname{TaC}\left(R_{\theta} r \Gamma, \mathbb{R}^{2}\right)=\operatorname{TaC}\left(\Gamma, \mathbb{R}^{2}\right)$, the $g_{m}$ 's are elementary functions and, for $F(d m)$ almost every $m=(b, r, \theta)$, writing $L=\mathcal{H}^{1}(\Gamma)=\operatorname{Per}(D)$, we have

$$
V_{g_{m}}\left(\mathbb{R}^{2}\right)=|b| r L \quad \text { and } \quad \operatorname{LTaC}\left(g_{m}, \mathbb{R}^{2}\right)=|b| \operatorname{TaC}\left(\Gamma, \mathbb{R}^{2}\right) .
$$

Let us remark that Equation (11) becomes

$$
\int_{\mathbb{R}^{d}} \int_{\mathbb{R}^{2}}\left|g_{m}(x)\right| d x F(d m)=\mathbb{E}(|B|) \bar{a}<+\infty .
$$

Equation (21) is easily checked since

$$
\begin{gathered}
\int_{\mathbb{R}^{d}} V_{g_{m}}\left(\mathbb{R}^{2}\right) F(d m)=\mathbb{E}(|B|) \mathbb{E}(R) L<+\infty \text { and } \\
\int_{\mathbb{R}^{d}} \operatorname{LTaC}_{g_{m}}\left(\mathbb{R}^{2}\right) F(d m)=\mathbb{E}(|B|) \operatorname{TaC}\left(\Gamma, \mathbb{R}^{2}\right)<+\infty .
\end{gathered}
$$

Assumption (22) also follows from the fact that $\bar{a}<+\infty$ and $\mathbb{E}(|B|)<+\infty$. For (24), let us remark that $\mathcal{I}_{g_{m}}=\emptyset$ and $\mathcal{C}_{g_{m}}=R_{\theta} r \mathcal{C}_{\Gamma}$ so that $\mathcal{H}^{0}\left(\mathcal{S}_{g_{m}} \backslash \mathcal{R}_{g_{m}}\right)=\mathcal{H}^{0}\left(\mathcal{C}_{\Gamma}\right)$, ensuring (24), by assumption on $\Gamma$.

Now, in order to check (25) and (26) we need a kind of kinematic formula. 
Lemma 1 (Kinematic formula). Let $f$ be a measurable non-negative function on $[-\pi, \pi)^{2}$, periodically extended to $\mathbb{R}^{2}$, and let $\Gamma_{1}, \Gamma_{2}$ be two piecewise regular Jordan (simple closed) curves. Let $I_{\Gamma_{1}, \Gamma_{2}}(f)$ be defined by

$$
I_{\Gamma_{1}, \Gamma_{2}}(f):=\int_{\mathbb{R}^{2}} \sum_{z \in \Gamma_{1} \cap \tau_{x} \Gamma_{2}} f\left(\operatorname{Arg} \nu_{\Gamma_{1}}(z), \operatorname{Arg} \nu_{\Gamma_{2}}(z-x)\right) d x .
$$

Then

$$
I_{\Gamma_{1}, \Gamma_{2}}(f)=\int_{0}^{L_{1}} \int_{0}^{L_{2}} f\left(\theta_{1}\left(s_{1}\right)+\frac{\pi}{2}, \theta_{2}\left(s_{2}\right)+\frac{\pi}{2}\right)\left|\sin \left(\theta_{1}\left(s_{1}\right)-\theta_{2}\left(s_{2}\right)\right)\right| d s_{1} d s_{2},
$$

where the curve $\Gamma_{1}$ (resp. $\Gamma_{2}$ ) of length $L_{1}=\mathcal{H}^{1}\left(\Gamma_{1}\right)$ (resp. $L_{2}=\mathcal{H}^{1}\left(\Gamma_{2}\right)$ ), is parametrized by $s_{1} \mapsto \gamma_{1}\left(s_{1}\right)$ (resp. by $s_{2} \mapsto \gamma_{2}\left(s_{2}\right)$ ) where $s_{1}$ (resp. $s_{2}$ ) is arc length, and $\theta_{1}\left(s_{1}\right)=\operatorname{Arg} \gamma_{1}^{\prime}\left(s_{1}\right)$ (resp. $\left.\theta_{2}\left(s_{2}\right)=\operatorname{Arg} \gamma_{2}^{\prime}\left(s_{2}\right)\right)$.

Proof. A heuristic proof of this result is given in the book of Santaló [31]. But it can be made fully rigorous using the co-area formula for Lipschitz mappings. Indeed, if $F: \mathbb{R}^{2} \mapsto \mathbb{R}^{2}$ is Lipschitz, then for any non-negative measurable function $v$ on $\mathbb{R}^{2}$, the co-area formula (see [20] or [7]) states that

$$
\int_{\mathbb{R}^{2}} v(y)|\operatorname{det}(D F(y))| d y=\int_{\mathbb{R}^{2}}\left(\int_{F^{-1}(x)} v(t) \mathcal{H}^{0}(d t)\right) d x=\int_{\mathbb{R}^{2}} \sum_{t \in F^{-1}(x)} v(t) d x
$$

Now, we use this formula with $F$ given by $F\left(s_{1}, s_{2}\right)=\gamma_{1}\left(s_{1}\right)-\gamma_{2}\left(s_{2}\right)$ for $\left(s_{1}, s_{2}\right) \in\left[0, L_{1}\right] \times\left[0, L_{2}\right]$ (and extended to $\mathbb{R}^{2}$ by periodicity for instance), and $v$ given by $v\left(s_{1}, s_{2}\right)=f\left(\theta_{1}\left(s_{1}\right)+\frac{\pi}{2}, \theta_{2}\left(s_{2}\right)+\right.$ $\left.\frac{\pi}{2}\right) \mathbf{I}_{\left(s_{1}, s_{2}\right) \in\left[0, L_{1}\right] \times\left[0, L_{2}\right]}$. Since $\Gamma_{1}$ and $\Gamma_{2}$ are piecewise regular Jordan curves, they have only a finite number of corner points, and therefore $\gamma_{1}$ and $\gamma_{2}$ are differentiable except at a finite number of points, and we can compute at a regular point $y=\left(s_{1}, s_{2}\right)$

$$
|\operatorname{det}(D F(y))|=\left|\operatorname{det}\left(\gamma_{1}^{\prime}\left(s_{1}\right), \gamma_{2}^{\prime}\left(s_{2}\right)\right)\right|=\left|\sin \left(\theta_{1}\left(s_{1}\right)-\theta_{2}\left(s_{2}\right)\right)\right| \text {. }
$$

And we also notice that if $x \in \mathbb{R}^{2}$ and $t=\left(s_{1}, s_{2}\right) \in F^{-1}(x)$, then $x=\gamma_{1}\left(s_{1}\right)-\gamma_{2}\left(s_{2}\right)$ and therefore $z=\gamma_{1}\left(s_{1}\right)$ belongs to both $\Gamma_{1}$ and $\tau_{x} \Gamma_{2}$, and $\gamma_{2}\left(s_{2}\right)=z-x$. This concludes the proof of the lemma.

In particular, taking $f=1$, it follows that $I_{\Gamma_{1}, \Gamma_{2}}(1) \leq L_{1} L_{2}$. Note also that we moreover have the exact formula

$$
\int_{0}^{2 \pi} \int_{0}^{2 \pi} I_{R_{\theta} \Gamma_{1}, R_{\theta^{\prime}} \Gamma_{2}}(1) d \theta d \theta^{\prime}=2 \pi \times \int_{0}^{2 \pi}|\sin (\theta)| d \theta L_{1} L_{2}=2 \pi \times 4 L_{1} L_{2},
$$

according to a generalization of Poincaré's Formula (see [31] for instance). Using the fact that $\Gamma$ is a finite disjoint union of Jordan curves we obtain that $I_{R_{\theta} r \Gamma, R_{\theta^{\prime}} r^{\prime} \Gamma}(1) \leq L^{2} r r^{\prime}$, since the length of $R_{\theta} r \Gamma$, resp. $R_{\theta^{\prime}} r^{\prime} \Gamma$, is $r L$, resp. $r^{\prime} L$, with $L=\mathcal{H}^{1}(\Gamma)$ the length of $\Gamma$ and

$$
\int_{0}^{2 \pi} \int_{0}^{2 \pi} I_{R_{\theta} r \Gamma, R_{\theta^{\prime}} r^{\prime} \Gamma}(1) d \theta d \theta^{\prime}=2 \pi \times 4 L^{2} r r^{\prime} .
$$


It follows that

$$
\begin{aligned}
& \int_{\mathbb{R}^{d} \times \mathbb{R}^{d}} \int_{\mathbb{R}^{2}} \mathcal{H}^{0}\left(\mathcal{R}_{g_{m^{\prime}}} \cap \tau_{x} \mathcal{R}_{g_{m}}\right) d x F(d m) F\left(d m^{\prime}\right) \\
= & \int_{\mathbb{R}_{+}^{2}} \int_{[0,2 \pi]^{2}} I_{R_{\theta} r \Gamma, R_{\theta^{\prime}} r^{\prime} \Gamma}(1) F_{\Theta}(d \theta) F_{\Theta}\left(d \theta^{\prime}\right) F_{R}(d r) F_{R}\left(d r^{\prime}\right) \\
\leq & 4 L^{2}\left(\int_{\mathbb{R}^{+}} r F_{r}(d r)\right)^{2}=4 \bar{p}^{2},
\end{aligned}
$$

with $\bar{p}=L \mathbb{E}(R)$ the mean perimeter, proving (25).

Moreover, for $f\left(\theta_{1}, \theta_{2}\right)=\mathbf{I}_{\theta_{1} \equiv \theta_{2}}+\mathbf{I}_{\theta_{1} \equiv \theta_{2}+\pi}$, where $\equiv$ stands for equality modulo $2 \pi$, we clearly have

$$
I_{\Gamma_{1}, \Gamma_{2}}(f)=0
$$

in view of (38). Since $\Gamma$ is a finite disjoint union of Jordan curves, it follows that

$$
\begin{aligned}
& \int_{\mathbb{R}^{d} \times \mathbb{R}^{d}} \int_{\mathbb{R}^{2}} \mathcal{H}^{0}\left(\left\{y \in \mathcal{R}_{g_{m^{\prime}}} \cap \tau_{x} \mathcal{R}_{g_{m}} ; \nu_{g_{m^{\prime}}}(y)= \pm \nu_{g_{m}}(y-x)\right\}\right) d x F(d m) F\left(d m^{\prime}\right) \\
= & \int_{\mathbb{R}_{+}^{2}} \int_{[0,2 \pi]^{2}} I_{R_{\theta} r \Gamma, R_{\theta^{\prime}} r^{\prime} \Gamma}(f) F_{\Theta}(d \theta) F_{\Theta}\left(d \theta^{\prime}\right) F_{R}(d r) F_{R}\left(d r^{\prime}\right)=0,
\end{aligned}
$$

so that (26) holds. Therefore we get the statement of Theorem 4. Moreover, we also get

$$
\begin{aligned}
& \int_{\mathbb{R}^{d} \times \mathbb{R}^{d}} \int_{\mathbb{R}^{2}}\left(\left\|g_{m}\right\|_{\infty}+\left\|g_{m^{\prime}}\right\|_{\infty}\right) \mathcal{H}^{0}\left(\mathcal{R}_{g_{m^{\prime}}} \cap \tau_{x} \mathcal{R}_{g_{m}}\right) d x F(d m) F\left(d m^{\prime}\right) \\
\leq & 4 \bar{p}^{2} \int_{\mathbb{R} \times \mathbb{R}}\left(|b|+\left|b^{\prime}\right|\right) F_{B}(d b) F_{B}\left(d b^{\prime}\right)=8 \bar{p}^{2} \mathbb{E}(|B|)<+\infty,
\end{aligned}
$$

and (31) is also satisfied so that Theorem 5 holds.

Note that, we simply have

$$
\mathbb{E}\left(V_{X_{\Phi}}(U)\right)=\lambda \mathcal{L}(U) \int_{\mathbb{R}^{d}} V_{g_{m}}\left(\mathbb{R}^{2}\right) F(d m)=\lambda \mathcal{L}(U) \mathbb{E}(|B|) \mathbb{E}(R) \mathcal{H}^{1}(\Gamma),
$$

and

$$
\mathbb{E}\left(\operatorname{LTC}_{X_{\Phi}}(U)\right)=\lambda \mathcal{L}(U) \int_{\mathbb{R}^{d}} \operatorname{LTC}_{g_{m}}\left(\mathbb{R}^{2}\right) F(d m)=\lambda \mathcal{L}(U) \mathbb{E}(B) \operatorname{TC}\left(\Gamma, \mathbb{R}^{2}\right),
$$

using the fact that $\operatorname{LTC}_{g_{m}}\left(\mathbb{R}^{2}\right)=\left(b^{+}-b^{-}\right) \times \operatorname{sgn}(b) \times \mathrm{TC}\left(\Gamma, \mathbb{R}^{2}\right)=b \mathrm{TC}\left(\Gamma, \mathbb{R}^{2}\right)$. Since $\mathrm{TC}\left(\Gamma, \mathbb{R}^{2}\right)=$ $2 \pi \chi(D)$, we obtain the first general statement. For $u \neq 0$, we can explicitly compute the characteristic function of $X_{\Phi}(0)$, given by

$$
\mathbb{E}\left(e^{i u X_{\Phi}(0)}\right)=\exp \left(\lambda \int_{\mathbb{R}^{d} \times \mathbb{R}^{2}}\left(e^{i u g_{m}(x)}-1\right) F(d m) d x\right)=e^{\lambda \bar{a}\left(\widehat{F}_{B}(u)-1\right)},
$$

where $\widehat{F}_{B}(u)=\mathbb{E}\left(e^{i u B}\right)=\int_{\mathbb{R}} e^{i u b} F_{B}(d b)$ is the characteristic function of $B$. In particular for $B=1$ a.s. $X_{\Phi}(0)$ is a Poisson random variable of parameter $\lambda \bar{a}$. We also have, writing $h_{u}$ as previoulsy,

$$
\begin{aligned}
\operatorname{LP}_{g_{m}}\left(h_{u}, \mathbb{R}^{2}\right) & =\int_{b^{-}}^{b+} e^{i u t} \mathcal{H}^{1}\left(R_{\theta} r \Gamma\right) d t=r \mathcal{H}^{1}(\Gamma) \frac{e^{i u b^{+}}-e^{i u b^{-}}}{i u} \\
\operatorname{LTC}_{g_{m}}\left(h_{u}, \mathbb{R}^{2}\right) & =\int_{b^{-}}^{b^{+}} e^{i u t} \operatorname{sgn}(b) \operatorname{TC}\left(R_{\theta} r \Gamma, \mathbb{R}^{2}\right) d t=\operatorname{TC}\left(\Gamma, \mathbb{R}^{2}\right) \frac{e^{i u b^{+}}-e^{i u b^{-}}}{i u} \operatorname{sgn}(b),
\end{aligned}
$$


so that

$$
\int_{\mathbb{R}^{d}} \operatorname{LP}_{g_{m}}\left(h_{u}, \mathbb{R}^{2}\right) F(d m)=\mathbb{E}(R) \mathcal{H}^{1}(\Gamma) \frac{\widehat{F_{B^{+}}}(u)-\widehat{F_{B^{-}}}(u)}{i u}
$$

and

$$
\int_{\mathbb{R}^{d}} \operatorname{LTC}_{g_{m}}\left(h_{u}, \mathbb{R}^{2}\right) F(d m)=\operatorname{TC}\left(\Gamma, \mathbb{R}^{2}\right) \frac{\widehat{F_{B}}(u)-1}{i u}
$$

Therefore

$$
\overline{\mathrm{LP}_{X_{\Phi}}}\left(h_{u}\right)=\lambda \mathbb{E}\left(e^{i u X_{\Phi}(0)}\right) \mathbb{E}(R) \mathcal{H}^{1}(\Gamma) \frac{\widehat{F_{B^{+}}}(u)-\widehat{F_{B^{-}}}(u)}{i u} .
$$

Finally, let us remark that for $f\left(\theta_{1}, \theta_{2}\right)=\min \left(\left|\theta_{1}-\theta_{2}\right|, 2 \pi-\left|\theta_{1}-\theta_{2}\right|\right)$ (distance between two angles) and $f_{\pi}\left(\theta_{1}, \theta_{2}\right)=f\left(\pi+\theta_{1}, \theta_{2}\right)$, we get

$$
\begin{aligned}
& \int_{\mathbb{R}^{d}} \int_{\mathbb{R}^{2} \times \mathbb{R}^{d}}\left(e^{i u g_{m^{\prime}}^{+}(z)}-e^{i u g_{m^{\prime}}^{-}(z)}\right)\left(\int_{g_{m}^{-}(z-x)}^{g_{m}^{+}(z-x)} e^{i u s} d s\right) \\
\times & \sum_{z \in \tau_{x} \mathcal{R}_{g_{m}} \cap \mathcal{R}_{g_{m^{\prime}}}} d_{S^{1}}\left(\nu_{g_{m}}(z-x), \nu_{g_{m^{\prime}}}(z)\right) d x F(d m) F\left(d m^{\prime}\right) \\
= & \frac{\left(\int_{0}^{+\infty}\left(e^{i u b}-1\right) F_{B}(d b)\right)^{2}+\left(\int_{-\infty}^{0}\left(1-e^{i u b}\right) F_{B}(d b)\right)^{2}}{i u} \\
\times & \int_{\mathbb{R}_{+}^{2}} \int_{[0,2 \pi]^{2}} I_{R_{\theta} r \Gamma, R_{\theta^{\prime}} r^{\prime} \Gamma}(f) F_{\Theta}(d \theta) F_{\Theta}\left(d \theta^{\prime}\right) F_{R}(d r) F_{R}\left(d r^{\prime}\right) \\
+\quad & 2 \frac{\left(\int_{0}^{+\infty}\left(e^{i u b}-1\right) F_{B}(d b)\right)\left(\int_{-\infty}^{0}\left(1-e^{i u b^{\prime}}\right) F_{B}\left(d b^{\prime}\right)\right)}{i u} \\
\times \quad & \int_{\mathbb{R}_{+}^{2}} \int_{[0,2 \pi]^{2}} I_{R_{\theta} r \Gamma, R_{\theta^{\prime}} r^{\prime} \Gamma}\left(f_{\pi}\right) F_{\Theta}(d \theta) F_{\Theta}\left(d \theta^{\prime}\right) F_{R}(d r) F_{R}\left(d r^{\prime}\right)
\end{aligned}
$$

This last expression may not be simple to compute. However, assuming from now on that $F_{\Theta}(d \theta)=\frac{1}{2 \pi} \mathbf{I}_{[0,2 \pi]} d \theta$, we obtain by (38), for two Jordan curves $\Gamma_{1}, \Gamma_{2}$,

$$
\begin{aligned}
& \int_{[0,2 \pi]^{2}} I_{R_{\theta} \Gamma_{1}, R_{\theta^{\prime}} \Gamma_{2}}(f) F_{\Theta}(d \theta) F_{\Theta}\left(d \theta^{\prime}\right)=\frac{1}{2 \pi} \int_{0}^{2 \pi} \int_{0}^{L_{1}} \int_{0}^{L_{2}}\left|\sin \left(\theta_{1}\left(s_{1}\right)-\theta_{2}\left(s_{2}\right)-\theta\right)\right| \\
& \times \min \left(\left|\theta_{1}\left(s_{1}\right)-\theta_{2}\left(s_{2}\right)-\theta\right|, 2 \pi-\left|\theta_{1}\left(s_{1}\right)-\theta_{2}\left(s_{2}\right)-\theta\right|\right) d s_{1} d s_{2} d \theta \\
= & L_{1} L_{2} .
\end{aligned}
$$

Similarly we have

$$
\int_{[0,2 \pi]^{2}} I_{R_{\theta} \Gamma_{1}, R_{\theta^{\prime}} \Gamma_{2}}\left(f_{\pi}\right) F_{\Theta}(d \theta) F_{\Theta}\left(d \theta^{\prime}\right)=L_{1} L_{2} .
$$

Since $\Gamma$ is a finite disjoint union of such Jordan curves, we get

$$
\int_{[0,2 \pi]^{2}} I_{R_{\theta} r \Gamma, R_{\theta^{\prime}} r^{\prime} \Gamma}(f) F_{\Theta}(d \theta) F_{\Theta}\left(d \theta^{\prime}\right)=\int_{[0,2 \pi]^{2}} I_{R_{\theta} r \Gamma, R_{\theta^{\prime}} r^{\prime} \Gamma}\left(f_{\pi}\right) F_{\Theta}(d \theta) F_{\Theta}\left(d \theta^{\prime}\right)=L^{2} r r^{\prime} .
$$

It follows that for uniform rotations, we have

$$
\overline{\operatorname{LTC}_{X_{\Phi}}}\left(h_{u}\right)=\lambda \mathbb{E}\left(e^{i u X_{\Phi}(0)}\right) \frac{1}{i u}\left(\left(\widehat{F_{B}}(u)-1\right) \mathrm{TC}\left(\Gamma, \mathbb{R}^{2}\right)+\frac{\lambda}{2}\left(\widehat{F_{B^{+}}}(u)-\widehat{F_{B^{-}}}(u)\right)^{2} \bar{p}^{2}\right) .
$$


When moreover $B=1$ a.s., $B^{+}=1$ and $B^{-}=0$ a.s., we can deduce an exact formula for the mean level total curvature function of $X_{\Phi}$. Actually, in this case $X_{\Phi}(0)$ follows a Poisson law of parameter $\lambda \bar{a}$ and $\frac{\widehat{F_{B}}(u)-\widehat{F_{B}}(u)}{i u}=\frac{\widehat{F_{B}}(u)-1}{i u}$ is the characteristic function of a uniform random variable $Z$ on $[0,1]$, while $\widehat{F_{B}}(u) \frac{\widehat{F_{B}}(u)-1}{i u}$ is the characteristic function of $Z+1$. Then, considering $Z$ independent from $X_{\Phi}(0)$, we can write

$$
\begin{gathered}
\overline{\operatorname{LP}_{X_{\Phi}}}\left(h_{u}\right)=\lambda \mathbb{E}\left(e^{i u\left[X_{\Phi}(0)+Z\right]}\right) \mathbb{E}(R) \mathcal{H}^{1}(\Gamma), \text { and } \\
\overline{\operatorname{LTC}_{X_{\Phi}}}\left(h_{u}\right)=\lambda\left[\mathbb{E}\left(e^{i u\left[X_{\Phi}(0)+Z\right]}\right)\left(\mathrm{TC}\left(\Gamma, \mathbb{R}^{2}\right)-\frac{\lambda}{2} \bar{p}^{2}\right)+\mathbb{E}\left(e^{i u\left[X_{\Phi}(0)+Z+1\right]}\right) \frac{\lambda}{2} \bar{p}^{2}\right] .
\end{gathered}
$$

Note also that since here the random field $X_{\Phi}$ has integer values, then $\forall k \in \mathbb{N}$,

$$
\forall t \in(k, k+1], \quad\left\{x \in U ; X_{\Phi}(x) \geq t\right\}=\left\{x \in U ; X_{\Phi}(x) \geq k+1\right\},
$$

and therefore

$$
\overline{\operatorname{Per}\left(E_{X_{\Phi}}(t)\right)}=\overline{\operatorname{Per}\left(E_{X_{\Phi}}(k+1)\right)} \quad \text { and } \quad \overline{\operatorname{TC}\left(\partial E_{X_{\Phi}}(t)\right)}=\overline{\operatorname{TC}\left(\partial E_{X_{\Phi}}(k+1)\right)} .
$$

Hence we can conclude that $\forall k \in \mathbb{N}, \forall t \in(k, k+1]$

$$
\begin{aligned}
\overline{\operatorname{Per}\left(E_{X_{\Phi}}(t)\right)}=\lambda e^{-\lambda \bar{a}} \frac{(\lambda \bar{a})^{k}}{k !} \bar{p} & \text { and } \\
& \overline{\operatorname{TC}\left(\partial E_{X_{\Phi}}(t)\right)}=\lambda e^{-\lambda \bar{a}} \frac{(\lambda \bar{a})^{k}}{k !}\left(\operatorname{TC}\left(\Gamma, \mathbb{R}^{2}\right)-\frac{\lambda}{2} \bar{p}^{2}+\frac{\bar{p}^{2}}{2 \bar{a}} k\right) .
\end{aligned}
$$

Note that, thanks to Gauss-Bonnet Theorem, we have $\mathrm{TC}\left(\Gamma, \mathbb{R}^{2}\right)=2 \pi \chi(D)$, so that we can rewrite this as in (37).

Let us remark that Formula (37) only involves the Euler Characteristic, the mean perimeter and the mean area of the shapes. When $k=0$, we find the formula of the mean Euler Characteristic density of a rotation invariant Boolean model as obtained by Mecke and Wagner in [28] and by Mecke in [27] stating that

$$
\lim _{r \rightarrow+\infty} \frac{\mathbb{E}\left(\chi\left(\left\{x \in r U ; X_{\Phi}(x) \geq 1\right\}\right)\right)}{\pi \mathcal{L}(r U)}=e^{-\lambda m_{0}(D)}\left(\lambda m_{2}(D)-\lambda^{2} m_{1}(D)^{2}\right),
$$

with $m_{0}(D)=\bar{a}, m_{1}(D)=\bar{p} / 2 \pi$ and $m_{2}(D)=\chi(D) / \pi$. Actually, following the framework of [33] for Boolean models, we can define some volume densities for excursion sets as, for all $k \in \mathbb{N}$ and $t \in(k, k+1]$,

$$
\begin{aligned}
\overline{\mathcal{L}\left(E_{X_{\Phi}}(t)\right)} & :=\mathbb{P}(X(0) \geq k+1)=1-e^{-\lambda \bar{a}} \sum_{l=0}^{k} \frac{(\lambda \bar{a})^{l}}{l !} \\
\overline{\operatorname{Per}\left(E_{X_{\Phi}}(t)\right)} & =e^{-\lambda \bar{a}} \frac{(\lambda \bar{a})^{k}}{k !} \lambda \bar{p} \\
\overline{\chi\left(E_{X_{\Phi}}(t)\right)} & =\lambda e^{-\lambda \bar{a}} \frac{(\lambda \bar{a})^{k}}{k !}\left(\chi(D)-\frac{\lambda}{4 \pi} \bar{p}^{2}+\frac{\bar{p}^{2}}{4 \pi \bar{a}} k\right),
\end{aligned}
$$

recovering the results of Boolean model for $k=0$ in dimension 2 (see p.389 of [33]).

The typical behavior of $\overline{\chi\left(E_{X_{\Phi}}(k)\right)}$, as a function of $k \in \mathbb{N}$, is the following: 
- It starts, when $k$ is small, by being negative. This is explained by the fact that $\left\{X_{\Phi} \geq k\right\}$ is essentially made of one big connected component with many small holes in it. In particular the minimum value of $\overline{\chi\left(E_{X_{\Phi}}(k)\right)}$ is achieved for an integer denoted $k_{-}$. The explicit value of $k_{-}$can be computed from Equation (39). The formula is not very nice, but it has a simple asymptotic behavior when $\lambda$ is large, since then we have

$$
k_{-}=\lambda \bar{a}-\sqrt{\lambda \bar{a}}+O(1) .
$$

- Then, after $k_{-}$, the density $\overline{\chi\left(E_{X_{\Phi}}(k)\right)}$ increases and it crosses 0 in the interval that contains $k_{0}$ where

$$
k_{0}=\lambda \bar{a}-\frac{2 \pi \bar{a}}{\bar{p}^{2}} \chi(D)=\lambda \bar{a}+O(1) .
$$

For this level, there are as many connected components as holes.

- After $k_{0}$, the density $\overline{\chi\left(E_{X_{\Phi}}(k)\right)}$ is positive and it increases till a value $k_{+}$and afterwards it decreases and goes to 0 as $k$ goes to infinity. As for $k_{-}$the value of $k_{+}$is explicit, and its asymptotic behavior when $\lambda$ is large is

$$
k_{+}=\lambda \bar{a}+\sqrt{\lambda \bar{a}}+O(1) .
$$

\section{Example 1: Random disks}

We assume here that $D=D(0,1)$ is a disk of radius 1 , and that $B=1$ a.s. In this case we have $\mathrm{TC}(\partial D)=2 \pi \chi(D)=2 \pi, \bar{p}=2 \pi \mathbb{E}(R)$ and $\bar{a}=\pi \mathbb{E}\left(R^{2}\right)$. Note also that since $R_{\theta} r D=r D$ for all $\theta$, whatever $F_{\Theta}$ is, the shot noise random field has the same law as the one with marks given by $\tilde{m}=(b, r) \in[0,+\infty)^{2} \subset \mathbb{R}^{2}$, with distribution $G(d \tilde{m})=F_{B}(d b) F_{R}(d r)$. An example of such a random field with comparisons between the theoretical value of $\mathbb{E}\left(\mathrm{TC}\left(\partial E_{X_{\phi}}(t), U\right)\right) / 2 \pi$ of Euler Characteristic and an empirical estimate on a large domain are shown on Figure 7 . The caption of the figure gives the practical and technical details of the simulation.

Let us remark that we can also compute the mean level total curvature for a non isotropic shape. This is the case of squares for instance, as developed in the following example.

\section{Example 2: Random squares}

We assume here that $D$ is a square of side length 1 and $\Theta=0$ a.s. with $F(d m)=F_{B}(d b) F_{R}(d r) \delta_{0}(d \theta)$ or equivalently that marks are given by $\tilde{m}=(b, r) \in \mathbb{R} \times[0,+\infty) \subset \mathbb{R}^{2}$, with distribution $G(d \tilde{m})=F_{B}(d b) F_{R}(d r)$. In this case, $\Gamma=\partial D$ is made of four line segments, with $\mathrm{TC}\left(\Gamma, \mathbb{R}^{2}\right)=$ $2 \pi \chi(D)=2 \pi, \bar{p}=4 \mathbb{E}(R)$ and $\bar{a}=\mathbb{E}\left(R^{2}\right)$. On the boundary of a square, the curvature is 0 , and it has four corner points with a turning angle equal to $\pi / 2$. Now, considering (36), we get for $u \neq 0$ and $h_{u}(t)=e^{i u t}$,

$$
\int_{\mathbb{R}^{d}} \operatorname{LTC}_{g_{m}}\left(h_{u}\right) F(d m)=\operatorname{TC}\left(\Gamma, \mathbb{R}^{2}\right) \frac{\widehat{F_{B}}(u)-1}{i u} .
$$

For the second term, according to the kinematic formula (38), the only remaining terms are for $\theta_{1}\left(s_{1}\right)=\theta_{2}\left(s_{2}\right) \pm \frac{\pi}{2}$ for which

$$
f\left(\theta_{1}\left(s_{1}\right), \theta_{2}\left(s_{2}\right)\right)=\min \left(\left|\theta_{1}\left(s_{1}\right)-\theta_{2}\left(s_{2}\right)\right|, 2 \pi-\left|\theta_{1}\left(s_{1}\right)-\theta_{2}\left(s_{2}\right)\right|\right)=\frac{\pi}{2}=f_{\pi}\left(\theta_{1}\left(s_{1}\right), \theta_{2}\left(s_{2}\right)\right) .
$$

It follows that

$$
I_{\Gamma_{1}, \Gamma_{2}}(f)=\frac{\pi}{2} \times 8 r_{1} \times r_{2}=I_{\Gamma_{1}, \Gamma_{2}}\left(f_{\pi}\right),
$$

and therefore

$$
\int_{\mathbb{R}^{2}} \sum_{z \in \tau_{x} \mathcal{R}_{g_{m}} \cap \mathcal{R}_{g_{m^{\prime}}}} d_{S^{1}}\left(\nu_{g_{m}}(z-x), \nu_{g_{m^{\prime}}}(z)\right) d x=\frac{\pi}{2} \times 8 r \times r^{\prime}
$$



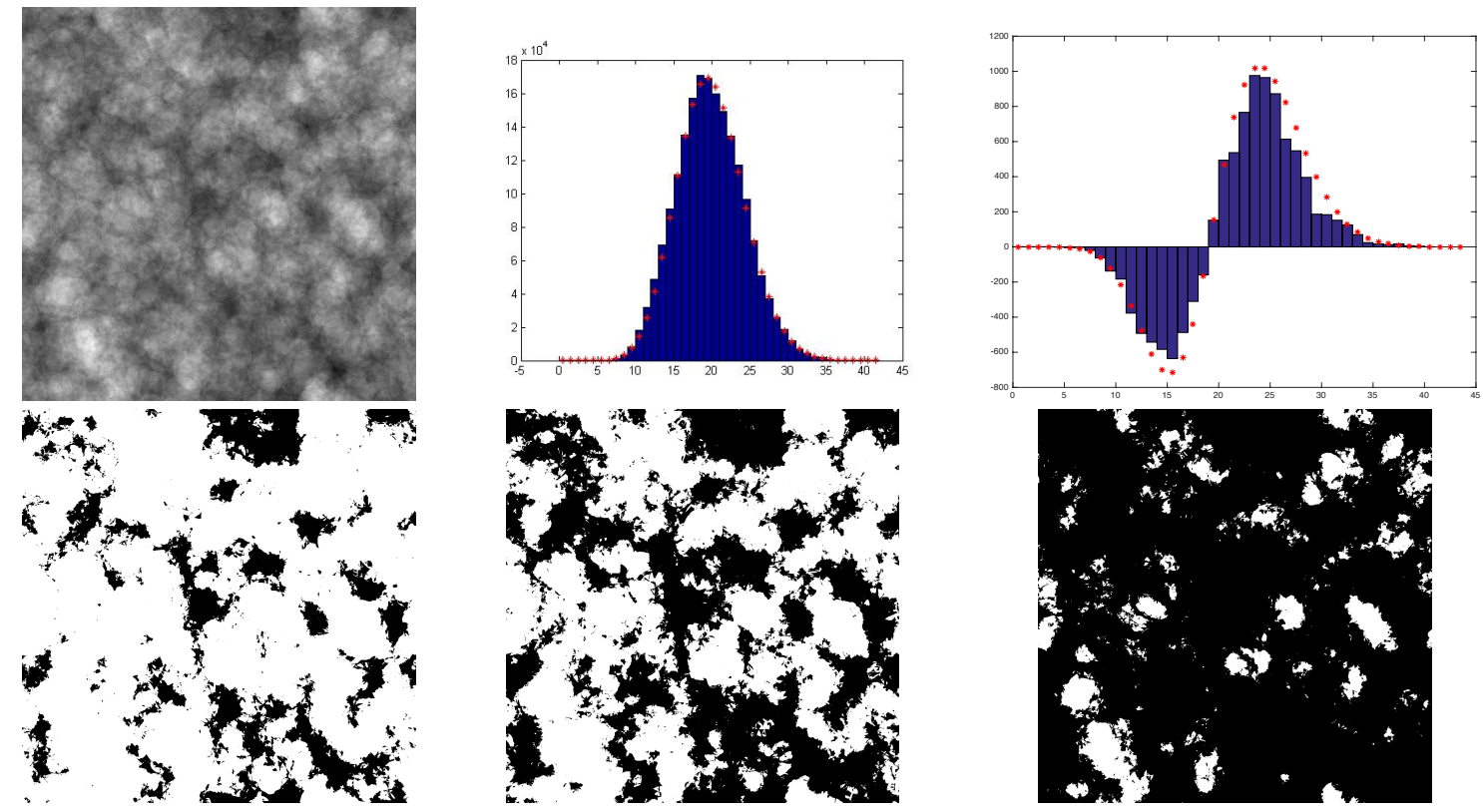

FIG 7. Shot noise random field with indicator functions of random disks. This sample has been obtained using Matlab, with a domain of size $2000 \times 2000$ pixels, a Poisson point process of intensity $\lambda=0.001$, and random disks of radius $R=50$ or $R=100$ (each with probability 0.5). Top middle and right figure: empirical Perimeter and Euler Characteristic as a function of the level $t$ (computed thanks to the Matlab functions bwperim and bweuler), compared with the theoretical values (red stars) of Equation (37). Bottom line: Three excursion sets corresponding respectively from left to right to the level $t=15, t=19$ (that is the "critical level" where the Euler Characteristic turns from negative to positive) and $t=25$.

Hence,

$$
\begin{aligned}
& \quad \frac{1}{2} \int_{\mathbb{R}^{d}} \int_{\mathbb{R}^{2} \times \mathbb{R}^{d}} \sum_{z \in \tau_{x} \mathcal{R}_{g_{m}} \cap \mathcal{R}_{g_{m^{\prime}}}}\left(e^{i u g_{m^{\prime}}^{+}(z)}-e^{i u g_{m^{\prime}}^{-}(z)}\right) \\
& \quad \times\left(\int_{g_{m}^{-}(z-x)}^{g_{m}^{+}(z-x)} e^{i u s} d s\right) d_{S^{1}}\left(\nu_{g_{m}}(z-x), \nu_{g_{m^{\prime}}}(z)\right) d x F(d m) F\left(d m^{\prime}\right) \\
& =\frac{\left(\widehat{F_{B^{+}}}(u)-\widehat{F_{B}}(u)\right)^{2}}{i u} 2 \pi \int_{\mathbb{R}_{+}^{2}} r r^{\prime} F_{R}(d r) F_{R}\left(d r^{\prime}\right)=2 \pi \frac{\left(\widehat{F_{B^{+}}}(u)-\widehat{F_{B^{-}}}(u)\right)^{2}}{i u} \frac{\bar{p}^{2}}{16} .
\end{aligned}
$$

Finally, in this case

$$
\begin{aligned}
& \mathbb{E}\left(\operatorname{LTC}_{X_{\Phi}}\left(h_{u}, U\right)\right) \\
= & \lambda \mathcal{L}(U) \mathbb{E}\left(e^{i u X_{\Phi}(0)}\right) \frac{1}{i u}\left(\left(\widehat{F_{B}}(u)-1\right) \mathrm{TC}\left(\Gamma, \mathbb{R}^{2}\right)+2 \pi \lambda\left(\widehat{F_{B^{+}}}(u)-\widehat{F_{B^{-}}}(u)\right)^{2} \frac{\bar{p}^{2}}{16}\right),
\end{aligned}
$$

and it corresponds to $\int_{\mathbb{R}} e^{i u t} \mathbb{E}\left(\mathrm{TC}\left(\partial E_{X_{\Phi}}(t), U\right)\right) d t$.

For $B=1$ a.s., taking the inverse Fourier transform as previously, we obtain

$\forall k \in \mathbb{N}, \forall t \in(k, k+1], \quad \frac{1}{2 \pi} \mathbb{E}\left(\mathrm{TC}\left(\partial E_{X_{\Phi}}(t), U\right)\right)=\lambda \mathcal{L}(U) e^{-\lambda \bar{a}} \frac{(\lambda \bar{a})^{k}}{k !}\left(1-\frac{\lambda}{16} \bar{p}^{2}+\frac{\bar{p}^{2}}{16 \bar{a}} k\right)$ 

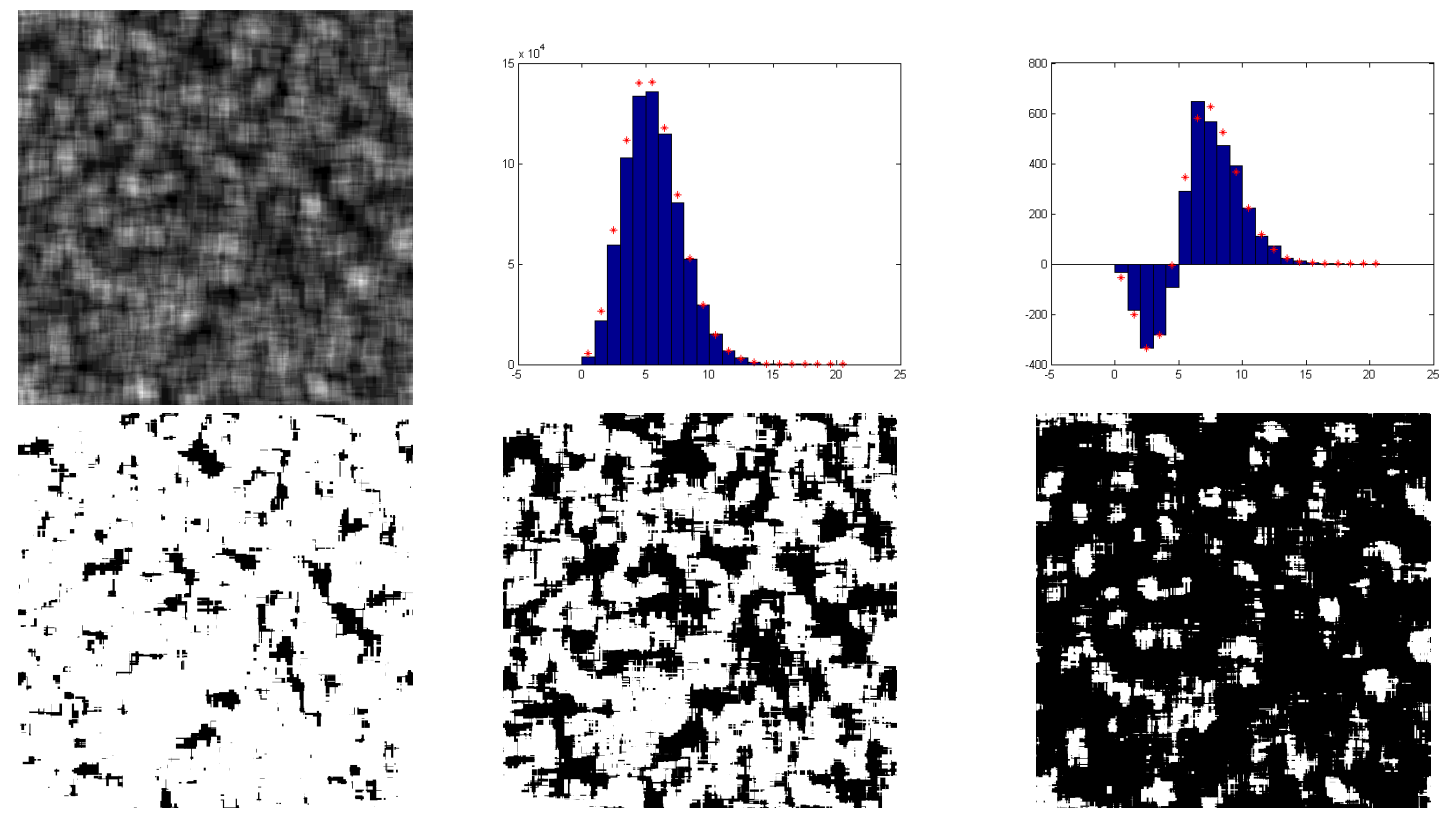

FIG 8. Shot noise random field with indicator functions of random squares. This sample has been obtained on a domain of size $2000 \times 2000$ pixels, with a Poisson point process of intensity $\lambda=0.005$, and random squares of fixed side length $R=100$. Top middle and right figures: empirical Perimeter and Euler Characteristic as functions of the level $t$, compared with the theoretical value (red stars) of Equation (40). Bottom line: Three excursion sets corresponding respectively from left to right to the level $t=3, t=5$ (that is the "critical level" where the Euler Characteristic turns from negative to positive) and $t=8$

It is illustrated on Figure 8. This formula generalizes one of the results of Decreusefond et al. [15]. Indeed, considering the Boolean model made of squares of constant size $R=2 \varepsilon$ a.s. for some $\varepsilon>0$, we get for $k=0$, and $a>0$,

$$
\forall t \in(0,1], \quad \frac{1}{2 \pi} \mathbb{E}\left(\mathrm{TC}\left(\partial E_{X_{\Phi}}(t),(0, a)^{2}\right)\right)=\lambda a^{2} e^{-\lambda(2 \varepsilon)^{2}}\left(1-\lambda(2 \varepsilon)^{2}\right),
$$

that corresponds to the mean Euler Characteristic of the Boolean model in dimension 2, considered in the torus of size $a>0$ in Theorem 11 of [15].

\section{Appendix}

\subsection{Proof of Proposition 2}

For the first point we will use the fact that for all $y \in \mathbb{R}$,

$$
\frac{2}{\pi} \int_{0}^{+\infty} \frac{\sin (u y)}{u} d u:=\lim _{M \rightarrow+\infty} \frac{2}{\pi} \int_{0}^{M} \frac{\sin (u y)}{u} d u=\operatorname{sgn}(y),
$$

with $\operatorname{sgn}(y)=1$ if $y>0, \operatorname{sgn}(y)=-1$ if $y<0$ and $\operatorname{sgn}(y)=0$ for $y=0$. We also remark that $C=\sup _{A>0} \frac{2}{\pi}\left|\int_{0}^{A} \frac{\sin (u)}{u} d u\right|<+\infty$. Since for $\varepsilon \in(0,1]$ we have $\left|\sin \left(u X_{1}\right)\right| \leq\left|u X_{1}\right|^{\varepsilon}$ and 
$\mathbb{E}\left(|W|\left|X_{1}\right|^{1+\varepsilon}\right)<+\infty$, by Fubini Theorem,

$$
\frac{2}{\pi} \int_{0}^{M} \frac{1}{u} \mathbb{E}\left(W X_{1} \sin \left(u X_{1}\right)\right) d u=\mathbb{E}\left(W X_{1} \frac{2}{\pi} \int_{0}^{M} \frac{\sin \left(u X_{1}\right)}{u} d u\right) .
$$

But, as $M$ goes to $+\infty, \frac{2}{\pi} \int_{0}^{M} \frac{\sin \left(u X_{1}\right)}{u} d u$ converges to $\operatorname{sgn}\left(X_{1}\right)$ a.s. and, since $\left|\int_{0}^{M} \frac{\sin \left(u X_{1}\right)}{u} d u\right|=$ $\left|\int_{0}^{M\left|X_{1}\right|} \frac{\sin (u)}{u} d u\right|$, it is uniformly bounded by $C$. Hence the dominated convergence theorem allows to conclude that

$$
\lim _{M \rightarrow+\infty} \frac{2}{\pi} \int_{0}^{M} \frac{1}{u} \mathbb{E}\left(W X_{1} \sin \left(u X_{1}\right)\right) d u=\mathbb{E}\left(W X_{1} \operatorname{sgn}\left(X_{1}\right)\right)=\mathbb{E}\left(W\left|X_{1}\right|\right) .
$$

For the second point, we start with the following identity:

$$
\frac{x_{1} x_{2}}{x_{1}^{2}+x_{2}^{2}} \mathbf{1}_{x_{1}^{2}+x_{2}^{2}>0}=\frac{4}{\pi} \int_{0}^{+\infty} \int_{0}^{+\infty} \frac{v_{1} v_{2}}{\left(v_{1}^{2}+v_{2}^{2}\right)^{2}} \sin \left(v_{1} x_{1}\right) \sin \left(v_{2} x_{2}\right) d v_{1} d v_{2} .
$$

This identity can be proved using the fact that the Fourier transform (or more precisely the sine transform) of a Cauchy-Lorentz function (function of the form $x \mapsto 2 a /\left(x^{2}+a^{2}\right)$ ) is a two-sided exponential function (function of the form $y \mapsto \exp (-a|y|)$ ). Moreover we have the following uniform bound.

Lemma 2. For $M, M^{\prime}>0$ and $x_{1}, x_{2} \in \mathbb{R}$, let us define

$$
F\left(M, M^{\prime}, x_{1}, x_{2}\right)=\int_{0}^{M} \int_{0}^{M^{\prime}} \frac{v_{1} v_{2}}{\left(v_{1}^{2}+v_{2}^{2}\right)^{2}} \sin \left(v_{1} x_{1}\right) \sin \left(v_{2} x_{2}\right) d v_{1} d v_{2} .
$$

Then, there exists a finite constant $C>0$ such that

$$
\forall M, M^{\prime}>0, \forall x_{1}, x_{2} \in \mathbb{R}, \quad\left|F\left(M, M^{\prime}, x_{1}, x_{2}\right)\right|<C .
$$

Proof. Let $x_{1}, x_{2} \in \mathbb{R}$, and let us denote $x_{1}=\rho \cos \theta$ and $x_{2}=\rho \sin \theta$, with $\rho>0$ and $\theta \in$ $[0,2 \pi)$. In the integral that defines $F\left(M, M^{\prime}, x_{1}, x_{2}\right)$, we make a change of variable to use polar coordinates. For $\alpha \in\left[0, \frac{\pi}{2}\right]$ we denote $R_{\alpha}^{M, M^{\prime}}=\frac{M}{\cos \alpha}$ if $\alpha \leq \arccos \frac{M}{\sqrt{M^{2}+M^{\prime 2}}}$, and $R_{\alpha}^{M, M^{\prime}}=\frac{M^{\prime}}{\sin \alpha}$ if $\alpha \geq \arccos \frac{M}{\sqrt{M^{2}+M^{\prime 2}}}$. We then get

$$
\begin{aligned}
F\left(M, M^{\prime}, x_{1}, x_{2}\right) & =\int_{0}^{\frac{\pi}{2}} \int_{0}^{R_{\alpha}^{M, M^{\prime}}} \frac{r^{2} \cos \alpha \sin \alpha}{r^{4}} \sin (r \rho \cos \theta \cos \alpha) \sin (r \rho \cos \theta \cos \alpha) r d r d \alpha \\
& =\int_{0}^{\frac{\pi}{2}} \cos \alpha \sin \alpha \int_{0}^{\rho R_{\alpha}^{M, M^{\prime}}} \frac{1}{r} \sin (r \cos \theta \cos \alpha) \sin (r \cos \theta \cos \alpha) d r d \alpha \\
& =\int_{0}^{\frac{\pi}{2}} \cos \alpha \sin \alpha \int_{0}^{\rho R_{\alpha}^{M, M^{\prime}}} \frac{1}{2 r}[\cos (r \cos (\theta+\alpha))-\cos (r \cos (\theta-\alpha))] d r d \alpha
\end{aligned}
$$

Now, let $A>0$, and let $t \in[-1,1]$. We have, by an integration by part,

$$
\int_{1}^{A} \frac{1}{r} \cos (r t) d r=\frac{\sin (A t)}{A t}-\frac{\sin (t)}{t}+\int_{1}^{A} \frac{\sin (r t)}{r^{2} t} d r .
$$


Using the change of variable $(r \mapsto r|t|)$ and the fact that $|\sin (x)| \leq \min (|x|, 1)$, we can bound the above right-hand side integral by

$$
\left|\int_{1}^{A} \frac{\sin (r t)}{r^{2} t} d r\right| \leq \int_{|t|}^{|t| A} \frac{|\sin (r)|}{r^{2}} d r \leq \int_{|t|}^{1} \frac{1}{r} d r+\int_{1}^{+\infty} \frac{1}{r^{2}} d r=1-\log |t| .
$$

If $t^{\prime}$ is also in $[-1,1]$, using that, for all $r>0,\left|\cos (r t)-\cos \left(r t^{\prime}\right)\right| \leq r\left|t-t^{\prime}\right| \leq 2 r$, we get

$$
\int_{0}^{1} \frac{1}{2 r}\left|\cos (r t)-\cos \left(r t^{\prime}\right)\right| d r \leq 1 .
$$

Putting all this together we finally have:

$$
\begin{aligned}
\left|F\left(M, M^{\prime}, x_{1}, x_{2}\right)\right| & \leq \int_{0}^{\frac{\pi}{2}}\left(3-\frac{1}{2} \log |\cos (\theta+\alpha)|-\frac{1}{2} \log |\cos (\theta-\alpha)|\right) d \alpha \\
& \leq \frac{3 \pi}{2}-\frac{1}{2} \int_{0}^{\pi} \log |\sin (x)| d x<+\infty .
\end{aligned}
$$

Hence, for $M, M^{\prime}$ positive real numbers, let

$$
Y_{M, M^{\prime}}:=\frac{4}{\pi} \int_{0}^{M} \int_{0}^{M^{\prime}} \frac{v_{1} v_{2}}{\left(v_{1}^{2}+v_{2}^{2}\right)^{2}} \sin \left(v_{1} X_{1}\right) \sin \left(v_{2} X_{2}\right) d v_{1} d v_{2},
$$

that is bounded by $C$ according to the previous lemma. Since $\mathbb{E}\left(|W|\left|X_{1} X_{2}\right|^{\varepsilon}\right)<+\infty$, by Fubini Theorem

$$
\frac{4}{\pi} \int_{0}^{M} \int_{0}^{M^{\prime}} \frac{v_{1} v_{2}}{\left(v_{1}^{2}+v_{2}^{2}\right)^{2}} \mathbb{E}\left(W \sin \left(v_{1} X_{1}\right) \sin \left(v_{2} X_{2}\right)\right) d v_{1} d v_{2}=\mathbb{E}\left(W Y_{M, M^{\prime}}\right)
$$

We have that $Y_{M, M^{\prime}}$ converges a.s., as $M, M^{\prime}$ go to infinity, to

$$
Y:=\frac{4}{\pi} \int_{0}^{+\infty} \int_{0}^{+\infty} \frac{v_{1} v_{2}}{\left(v_{1}^{2}+v_{2}^{2}\right)^{2}} \sin \left(v_{1} X_{1}\right) \sin \left(v_{2} X_{2}\right) d v_{1} d v_{2}=\frac{X_{1} X_{2}}{X_{1}^{2}+X_{2}^{2}} \mathbf{1}_{X_{1}^{2}+X_{2}^{2}>0}
$$

Hence the result follows again from the dominated convergence theorem.

\subsection{Proof of Proposition 3}

As in the proof of Theorem 2, our assumption implies that $\mathbb{E}\left(W \bar{K} e^{i n \Theta} \mathbf{1}_{|J|>0}\right)=0$ for all $n \neq 2$. Let $g: 2 \pi \mathbb{T} \rightarrow \mathbb{R}$ be a continuous bounded $2 \pi$ periodic function. For $N \geq 1$ we denote by $S_{N}(g)(\theta)=\sum_{n=-N}^{N}\left(1-\frac{|n|}{N}\right) c_{n}(g) e^{i n \theta}$ the Fejer sum of order $N$ of $g$, where the Fourier coefficients are given by $c_{n}(g)=\frac{1}{2 \pi} \int_{0}^{2 \pi} e^{-i n \theta} g(\theta) d \theta$. We obtain that

$$
\mathbb{E}\left(W \bar{K} S_{N}(g)(\Theta) \mathbf{1}_{|J|>0}\right)=\left(1-\frac{2}{N}\right) c_{2}(g) \mathbb{E}\left(W \bar{K} e^{2 i \Theta} \mathbf{1}_{|J|>0}\right) .
$$

Since, $\left(S_{N}(g)\right)_{N}$ converges uniformly to $g$ that is bounded and $\mathbb{E}(|W K|)<+\infty$, it follows that $\mathbb{E}\left(W \bar{K} g(\Theta) \mathbf{1}_{|J|>0}\right)=c_{2}(g) \mathbb{E}\left(W \bar{K} e^{2 i \Theta} \mathbf{1}_{|J|>0}\right)$, by the dominated convergence theorem. Now 
for the second point, we also introduce the real and the imaginary part of $J, J_{1}=\Re(J)$ and $J_{2}=\Im(J)$, so that

$$
\sin (2 \Theta) \mathbf{1}_{|J|>0}=2 \frac{J_{1} J_{2}}{J_{1}^{2}+J_{2}^{2}} \mathbf{1}_{J_{1}^{2}+J_{2}^{2}>0} .
$$

According to the second point of Proposition 2, we have

$$
\begin{aligned}
\mathbb{E}\left(W \bar{K} \sin (2 \Theta) \mathbf{1}_{|J|>0}\right) & =\frac{8}{\pi} \int_{0}^{+\infty} \int_{0}^{+\infty} \frac{v_{1} v_{2}}{\left(v_{1}^{2}+v_{2}^{2}\right)^{2}} \mathbb{E}\left(W \bar{K} \sin \left(v_{1} J_{1}\right) \sin \left(v_{2} J_{2}\right)\right) d v_{1} d v_{2} \\
& =-\frac{8}{\pi} \int_{0}^{+\infty} \int_{0}^{+\infty} \frac{v_{1} v_{2}}{\left(v_{1}^{2}+v_{2}^{2}\right)^{2}} \mathbb{E}\left(W \bar{K} \cos \left(v_{1} J_{1}+v_{2} J_{2}\right)\right) d v_{1} d v_{2}
\end{aligned}
$$

using the fact that $\mathbb{E}\left(W \bar{K} \cos \left(v_{1} J_{1}\right) \cos \left(v_{2} J_{2}\right)\right)=\mathbb{E}\left(W(-\bar{K}) \cos \left(v_{1} J_{2}\right) \cos \left(v_{2} J_{1}\right)\right)$ by (10) for $\theta=\pi / 2$. By a change of variables in polar coordinates we obtain

$$
\begin{aligned}
\mathbb{E}\left(W \bar{K} \sin 2 \Theta \mathbf{1}_{|J|>0}\right) & =-\frac{4}{\pi} \int_{0}^{+\infty} \int_{0}^{\pi / 2} \mathbb{E}\left(W \bar{K} \cos \left(v \Re\left(J e^{-i \alpha}\right)\right) \sin (2 \alpha) d \alpha \frac{d v}{v}\right. \\
& =-\frac{4}{\pi} \int_{0}^{+\infty} \int_{0}^{\pi / 2} \mathbb{E}\left(W \bar{K} e^{-2 i \alpha} \cos (v \Re(J))\right) \sin (2 \alpha) d \alpha \frac{d v}{v} \\
& =i \int_{0}^{+\infty} \mathbb{E}(W \bar{K} \cos (v \Re(J))) \frac{d v}{v} .
\end{aligned}
$$

Acknowledgments: We would like to sincerely thank the reviewers for their valuable comments and challenging remarks that helped us to improve substantially this paper.

\section{References}

[1] R. J. Adler. The Geometry of Random Field. John Wiley \& Sons, 1981.

[2] R. J. Adler. On excursion sets, tube formulas and maxima of random fields. The Annals of Applied Probability, 10(1):1-74, 2000.

[3] R. J. Adler, G. Samorodnitsky, and J. E. Taylor. Excursion sets of three classes of stable random fields. Adv. in Appl. Probab., 42(2):293-318, 2010.

[4] R. J. Adler, G. Samorodnitsky, and J. E. Taylor. High level excursion set geometry for non-Gaussian infinitely divisible random fields. The Annals of Probability, 41(1):134-169, 2013.

[5] R. J. Adler, E. Subag, and J. E. Taylor. Rotation and scale space random fields and the Gaussian kinematic formula. Ann. Statist., 40(6):2910-2942, 2012.

[6] R. J. Adler and J. E. Taylor. Random fields and geometry. Springer Monographs in Mathematics. Springer, New York, 2007.

[7] L. Ambrosio, N. Fusco, and D. Pallara. Functions of bounded variation and free discontinuity problems. Oxford university press, 2000.

[8] F. Baccelli and B. Błaszczyszyn. Stochastic Geometry and Wireless Networks, Volume I — Theory, volume 3, No 3-4 of Foundations and Trends in Networking. NoW Publishers, 2009.

[9] Y. Baryshnikov and R. Ghrist. Euler integration over definable functions. Proc. Natl. Acad. Sci. USA, 107(21):9525-9530, 2010. 
[10] H. Biermé and A. Desolneux. Crossings of smooth shot noise processes. The Annals of Applied Probability, 22(6):2240-2281, 2012.

[11] H. Biermé and A. Desolneux. On the perimeter of excursion sets of shot noise random fields. The Annals of Probability, 44(1):521-543, 2016.

[12] M. Bilodeau and D. Brenner. Theory of multivariate statistics. Springer Texts in Statistics. Springer-Verlag, New York, 1999.

[13] O. Bobrowski and M. S. Borman. Euler integration of Gaussian random fields and persistent homology. Journal of Topology and Analysis, 4(1):49-70, 2012.

[14] S. N. Chiu, D. Stoyan, W. S. Kendall, and J. Mecke. Stochastic geometry and its applications. Wiley Series in Probability and Statistics. Wiley-Blackwell, 2013. Third Edition.

[15] L. Decreusefond, E. Ferraz, H. Randriambololona, and A. Vergne. Simplicial homology of random configurations. Advances in Applied Probability, 46(2):325-347, 2014.

[16] E. Di Bernardino, A. Estrade, and J. R. León. A test of Gaussianity based on the Euler Characteristic of excursion sets. Electron. J. Stat., 11(1):843-890, 2017.

[17] M.P. Do Carmo. Differential Geometry of Curves and Surfaces. Prentice-Hall, 1976.

[18] A. Erdélyi, W. Magnus, F. Oberhettinger, and F. G. Tricomi. Tables of integral transforms. Vol. I. McGraw-Hill Book Company, Inc., New York-Toronto-London, 1954. Based, in part, on notes left by Harry Bateman.

[19] A. Estrade and J. R. León. A central limit theorem for the Euler characteristic of a Gaussian excursion set. The Annals of Probability, 44(6):3849-3878, 2016.

[20] L.C. Evans and R.F. Gariepy. Measure theory and fine properties of functions. Studies in Advanced Mathematics. CRC Press, 1992.

[21] L. Heinrich and V. Schmidt. Normal convergence of multidimensional shot noise and rates of this convergence. Advances in Applied Probability, 17(4):709-730, 1985.

[22] M. Hirsch. Differential Topology. Springer-Verlag, 1976.

[23] R. Lachièze-Rey. Two-dimensional Kac-Rice formula. Application to shot noise processes excursions. Technical report, MAP5, Univ. Paris Descartes, 2016. https://arxiv.org/abs/1510.00502.

[24] R. Lachièze-Rey. Bicovariograms and Euler characteristic of random fields excursions. Stochastic Processes and their Applications, 2018.

[25] R. Lachièze-Rey. Bicovariograms and Euler characteristic of regular sets. Mathematische Nachrichten, 291(2-3):398-419, 2018.

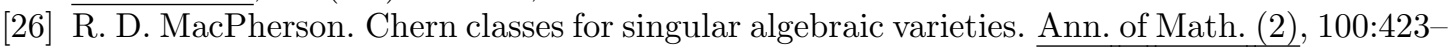
$432,1974$.

[27] K. R. Mecke. Exact moments of curvature measures in the Boolean model. Journal of Statistical Physics, 102(5-6):1343-1381, 2001.

[28] K. R. Mecke and H. Wagner. Euler characteristic and related measures for random geometric sets. Journal of Statistical Physics, 64(3-4):843-850, 1991.

[29] J.W. Milnor. On the total curvature of knots. The Annals of Mathematics, 52(2):248-257, 1950.

[30] I. Molchanov. Theory of Random Sets. Probability and its applications. Springer, 1st edition. edition, 2005.

[31] L.A. Santaló. Integral Geometry and Geometric Probability. Cambridge University Press, second edition edition, 2004.

[32] P. Schapira. Operations on constructible functions. J. Pure Appl. Algebra, 72(1):83-93, 1991.

[33] R. Schneider and W. Weil. Stochastic and integral geometry. Probability and its Applications. Springer-Verlag, Berlin, 2008. 
[34] D. Stoyan, W. S. Kendall, and J. Mecke. Stochastic geometry and its applications. Wiley Series in Probability and Mathematical Statistics: Applied Probability and Statistics. John Wiley \& Sons, Ltd., Chichester, 1987. With a foreword by D. G. Kendall.

[35] C. Thäle. 50 years sets with positive reach - a survey. Surv. Math. Appl., 3:123-165, 2008.

[36] O. Y. Viro. Some integral calculus based on Euler Characteristic. In Topology and geometry-Rohlin Seminar, volume 1346 of Lecture Notes in Math., pages 127-138. Springer, Berlin, 1988.

[37] K. J. Worsley. Local Maxima and the Expected Euler Characteristic of Excursion Sets of Chi2, F and t Fields. Advances in Applied Probability, 26:13-42, 31994.

[38] K. J. Worsley. The geometry of random images. Chance, 9(1):27-40, 1996. 